\title{
Eigenvalue problem for fully nonlinear second-order elliptic PDE on balls, II
}

\author{
Norihisa Ikoma ${ }^{1} \cdot$ Hitoshi Ishii ${ }^{2}$
}

Received: 2 October 2014 / Revised: 6 July 2015 / Accepted: 7 July 2015 / Published online: 25 July 2015 (C) The Author(s) 2015. This article is published with open access at SpringerLink.com

\begin{abstract}
This is a continuation of Ikoma and Ishii (Ann Inst H Poincaré Anal Non Linéaire 29:783-812,2012) and we study the eigenvalue problem for fully nonlinear elliptic operators, positively homogeneous of degree one, on finite intervals or balls. In the multi-dimensional case, we consider only radial eigenpairs. Our eigenvalue problem has a general first-order boundary condition which includes, as a special case, the Dirichlet, Neumann and Robin boundary conditions. Given a nonnegative integer $n$, we prove the existence and uniqueness, modulo multiplication of the eigenfunction by a positive constant, of an eigenpair whose eigenfunction, as a radial function in the multi-dimensional case, has exactly $n$ zeroes. When an eigenfunction has $n$ zeroes, we call the corresponding eigenvalue of $n$th order. Furthermore, we establish results concerning comparison of two eigenvalues, characterizations of $n$th order eigenvalues via differential inequalities, the maximum principle for the boundary value problem in connection with the principal eigenvalue, and existence of a solution having $n$ zeroes,
\end{abstract}

Communicated by Neil Trudinger.

Norihisa Ikoma was partially supported by JSPS Research Fellowships 24-2259. Hitoshi Ishii was supported in part by KAKENHI, \#23244015, \#23340028 and \#26220702, JSPS.

Bitoshi Ishii

hitoshi.ishii@waseda.jp

Norihisa Ikoma

ikoma@se.kanazawa-u.ac.jp

1 Faculty of Mathematics and Physics, Institute of Science and Engineering, Kanazawa University, Kakuma, Kanazawa, Ishikawa 9201192, Japan

2 Faculty of Education and Integrated Arts and Sciences, Waseda University, 1-6-1 Nishi-Waseda, Shinjuku-ku, Tokyo 169-8050, Japan 
as a radial function in the multi-dimensional case, of the boundary value problem with an inhomogeneous term.

Keywords Eigenvalue problem - Fully nonlinear equation - General boundary conditions · Principal eigenvalues · Higher order eigenvalues

\section{Contents}

1 Introduction . . . . . . . . . . . . . . . . . . . . . . . . . 452

2 Main results in the one-dimensional case . . . . . . . . . . . . . . . . . . . . . 458

3 Main results in the radial case . . . . . . . . . . . . . . . . . . . . . . . . . . 461

4 Preliminary observations and results in the one-dimensional case . . . . . . . . . . . . . 463

4.1 Two basic symmetries . . . . . . . . . . . . . . . . . . . . . . . . . . 463

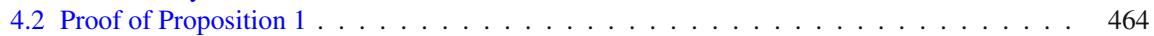

4.3 The strong maximum principle . . . . . . . . . . . . . . . . . . . . . . . 466

4.4 The maximum and comparison principles . . . . . . . . . . . . . . . . . . . . . . . . . 467

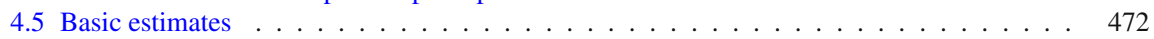

5 Proofs of the main results in the one-dimensional case . . . . . . . . . . . . . . . . . . . . 475

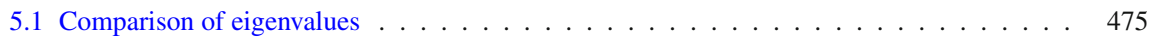

5.2 Existence of principal eigenpairs _ . . . . . . . . . . . . . . . . . . . . . . . 476

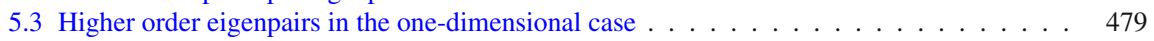

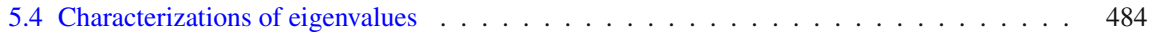

5.5 Inhomogeneous equations . . . . . . . . . . . . . . . . . . . . . . . . . . . . . . . . . . . . . . . . . . .

6 Preliminary observations in the radial case . . . . . . . . . . . . . . . . . . . . . . 496

7 Proofs of the main results in the radial case . . . . . . . . . . . . . . . . . . . 505

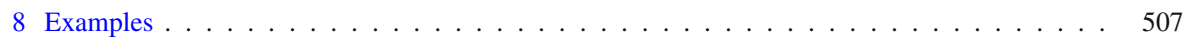

8.1 Non-uniqueness for $(18) \ldots \ldots \ldots \ldots \ldots$. . . . . . . . . . . . . . . . . . . . . . . . . . . . . . . . . . . . . . 507

8.2 Non-uniqueness for $(26) \ldots \ldots \ldots \ldots \ldots$. . . . . . . . . . . . . . . . . . . . . . . . . . . . . . . . . . . . 508

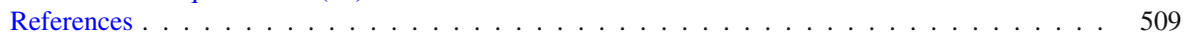

\section{Introduction}

This paper is a continuation of [25] and deals with the eigenvalue problem

$$
\begin{aligned}
& F\left(D^{2} u, D u, u, x\right)+\mu u=0 \quad \text { in } \Omega, \\
& B(D u, u, x)=0 \quad \text { on } \partial \Omega \text {. }
\end{aligned}
$$

Here $F: \mathbb{S}^{N} \times \mathbb{R}^{N} \times \mathbb{R} \times \Omega \rightarrow \mathbb{R}$ and $B: \mathbb{R}^{N} \times \mathbb{R} \times \partial \Omega \rightarrow \mathbb{R}$ are given functions, $\mathbb{S}^{N}$ denotes the set of all $N \times N$ real symmetric matrices, $\Omega \subset \mathbb{R}^{N}$ is an interval $(a, b)$ if $N=1$ and, otherwise, an open ball $B_{R}$ with radius $R>0$ centered at the origin, and $(\mu, u)$ represents an unknown pair of a real number and a function on $\Omega$ in a Sobolev space, which will be specified later.

For a function $u$ on $\Omega$ in a Sobolev space, if Eq. (1) holds in the almost everywhere sense, then we call $u$ a solution of (1).

We call a pair $(\mu, u)$ of a constant and a function on $\bar{\Omega}$ an eigenpair of (1)-(2) [resp. of (1)) if $(\mu, u)$ satisfies (1)-(2) [resp. (1)] and $u \neq \equiv 0$. When $(\mu, u)$ is an eigenpair, we call $\mu$ and $u$ an eigenvalue and an eigenfunction, respectively. For an eigenpair $(\mu, u)$, if either $u(x)>0$ in $\Omega$ or $u(x)<0$ in $\Omega$, then we call $(\mu, u)$ [resp. $\mu$ and $u$ ] a principal eigenpair [resp. a principal eigenvalue and a principal eigenfunction]. 
Furthermore, if $u(x)>0$ [resp. $u(x)<0$ ] in $\Omega$, then we call the eigenpair $(\mu, u)$ and the eigenfunction $u$ positive [resp. negative]. When $N \geq 2$, an eigenpair $(\mu, u)$ is called a radial eigenpair if $u$ is radially symmetric.

The eigenvalue problem for fully nonlinear PDE (1) has been paid much attention since the work of P.-L. Lions [28]. For the recent developments, we refer to [2,3,6$14,18,19,21,22,26,30-32]$. See also $[1,4,5,17,24]$ for the recent contributions and overviews on the eigenvalue problem for linear elliptic operators.

In [25], under the Dirichlet boundary condition, that is, in the case where the function $B$ is given by $B(p, u, x)=u$, the authors have proved the existence of sequences of eigenpairs [resp. radial eigenpairs] of (1)-(2) when $N=1$ [resp. $N \geq 2$ ] and that, modulo multiplication of eigenfunctions by positive constants, there is no other eigenpairs [resp. radial eigenpairs] of (1)-(2) when $N=1$ [resp. $N \geq 2$ ].

Our aim of this paper is to establish the existence of eigenpairs when $N=1$ and radial eigenpairs when $N \geq 2$ of the problem (1)-(2), and to provide basic properties of eigenpairs. We thus generalize the results in [25] to cover the eigenvalue problem (1) with general boundary conditions. The results due to Patrizi [30] concern the eigenvalue problem for (1) with the Robin boundary condition, and are related closely to our results in this paper.

Throughout this paper, as far as we are concerned with (1)-(2), we make the following assumptions on $F$. The conditions (F1)-(F4) below are the same as those in [25] except that the case of $(N, \Lambda)=(1, \infty)$ is excluded and an integrability requirement on the function $x \mapsto F(0,0,0, x)$ is added in (F2) below. See also Esteban, Felmer and Quaas [21] for a formulation of eigenvalue problems similar to the one below.

(F1) The function $F: \mathbb{S}^{N} \times \mathbb{R}^{N} \times \mathbb{R} \times \Omega \rightarrow \mathbb{R}$ is a Carathéodory function, i.e., the function $x \mapsto F(M, p, u, x)$ is measurable for any $(M, p, u) \in \mathbb{S}^{N} \times \mathbb{R}^{N+1}$ and the function $(M, p, u) \mapsto F(M, p, u, x)$ is continuous for a.e. $x \in \Omega$.

(F2) There exist constants $0<\lambda \leq \Lambda<\infty, q \in[1, \infty]$ and functions $\beta, \gamma \in$ $L^{q}(\Omega)$ such that

$$
\begin{aligned}
F\left(M_{1}, p_{1}, u_{1}, x\right)-F\left(M_{2}, p_{2}, u_{2}, x\right) \leq & P^{+}\left(M_{1}-M_{2}\right)+\beta(x)\left|p_{1}-p_{2}\right| \\
& +\gamma(x)\left|u_{1}-u_{2}\right|
\end{aligned}
$$

for all $\left(M_{1}, p_{1}, u_{1}\right),\left(M_{2}, p_{2}, u_{2}\right) \in \mathbb{S}^{N} \times \mathbb{R}^{N+1}$ and a.e. $x \in \Omega$. Furthermore, the function $x \mapsto F(0,0,0, x)$ belongs to $L^{q}(\Omega)$. For the definition of $P^{+}$, see below.

(F3) $F(t M, t p, t u, x)=t F(M, p, u, x)$ for all $t \geq 0$, all $(M, p, u) \in \mathbb{S}^{N} \times \mathbb{R}^{N+1}$ and a.e. $x \in \Omega$.

Here and in what follows $P^{ \pm}$denote the Pucci operators defined as the functions given by

$$
P^{+}(M)=\sup \left\{\operatorname{tr}(A M): A \in \mathbb{S}^{N}, \lambda I_{N} \leq A \leq \Lambda I_{N}\right\}
$$

and

$$
P^{-}(M)=\inf \left\{\operatorname{tr}(A M): A \in \mathbb{S}^{N}, \lambda I_{N} \leq A \leq \Lambda I_{N}\right\}
$$


where $I_{N}$ denotes the $N \times N$ identity matrix and the relation, $X \leq Y$, is the standard order relation between $X, Y \in \mathbb{S}^{N}$. For instance, if $N=1$, then $P^{+}(m)=\lambda m$ for $m \leq 0$ and $P^{+}(m)=\Lambda m$ for $m>0$.

Condition (F3) represents a characteristic of our eigenvalue problem, where every eigenpair $(\mu, u)$ is supposed to have the positive homogeneity property, that is, $(\mu, t u)$ is also an eigenpair for any $t>0$. This is the fundamental property in our eigenvalue problem, and a natural requirement on the function $B$ in (2) is then that $B(p, u, x)$ should be positively homogeneous of degree one in the variables $(p, u)$. Furthermore, remark that (F3) implies $F(0,0,0, x)=0$ a.e. in $\Omega$.

Instead of using the equation $B(D u, u, x)=0$, we actually use the membership relation (differential inclusions) to describe the boundary condition of our eigenvalue problem, which is a more suitable treatment of the boundary condition having the positive homegeneity.

Before introducing this relation, we make the following observation in the case when $N=1$. Let $u \in C^{1}([a, b])$ and consider the situation where $u(x)>0$ for all $x \in(a, c)$ and some $c \in(a, b)$, and $\left(u(a), u^{\prime}(a)\right) \neq(0,0)$ (Fig. 1). We have two cases: (1) $u(a)>0$, or $(2) u(a)=0$ and $u^{\prime}(a)>0$. The set of points $(\xi, \eta):=\left(-u^{\prime}(a), u(a)\right)$ in $\mathbb{R}^{2}$, where $u$ ranges over all such functions $u$, is the half-plane (Fig. 2) given by

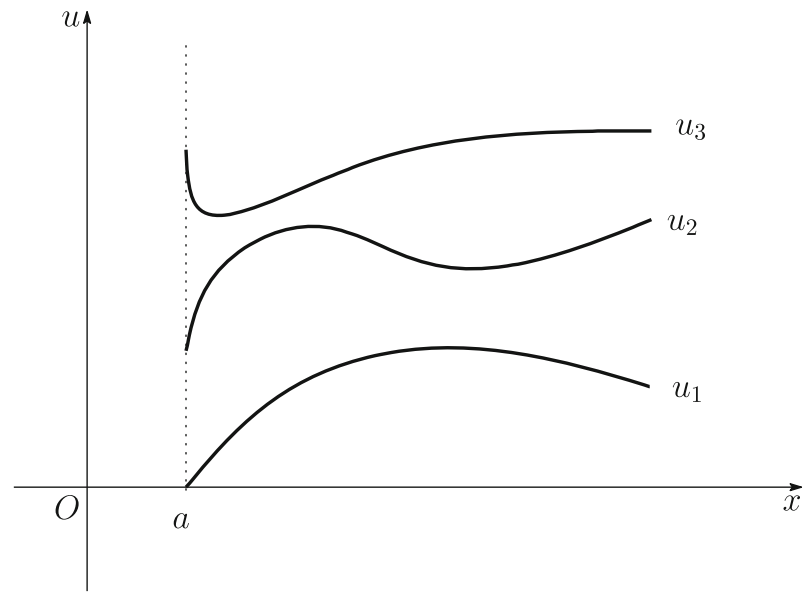

Fig. 1 Three typical behaviors near $x=a$

Fig. 2 The half-plane $H^{+}$

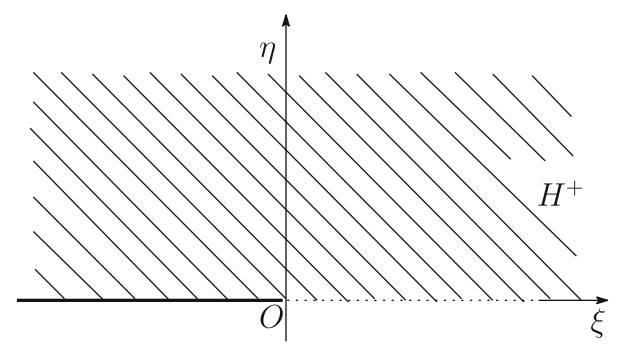


Fig. 3 Half-line $l(\theta)$

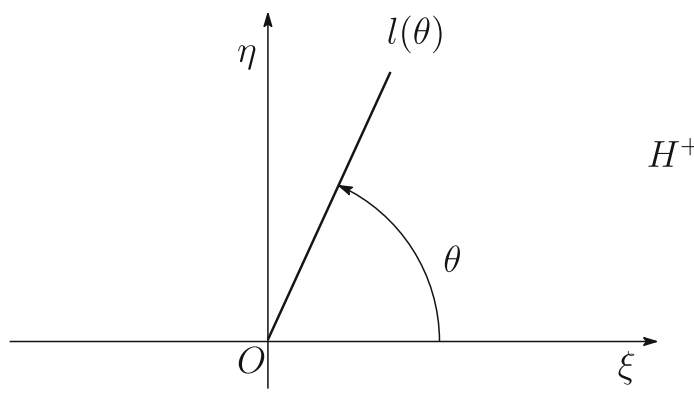

$$
H^{+}:=\mathbb{R} \times(0, \infty) \cup(-\infty, 0) \times\{0\} .
$$

We set

$$
H^{-}:=-H^{+}=\mathbb{R} \times(-\infty, 0) \cup(0, \infty) \times\{0\},
$$

and note that $H^{-}$is the set of points $\left(-u^{\prime}(a), u(a)\right)$, where $u$ ranges over all $u \in$ $C^{1}([a, b])$ such that $\left(u(a), u^{\prime}(a)\right) \neq(0,0)$ and $u(x)<0$ in $(a, c)$ for some $c \in(a, b)$. Note also that the punctured plane $\mathbb{R}^{2} \backslash\{(0,0)\}$ is the disjoint union of the half-planes $\mathrm{H}^{+}$and $\mathrm{H}^{-}$. We observe in view of the polar coordinates in the plane that any open half-line $l$ in $H^{+}$with vertex at the origin can be parametrized by the angle $\theta \in(0, \pi]$. That is, such an open half-line $l$ can be described by a constant $\theta \in(0, \pi]$ as

$$
l=\{(r \cos \theta, r \sin \theta): r>0\},
$$

which we denote by $l(\theta)$ (Fig. 3).

Similarly, any open half-line with vertex at the origin in $H^{-}$is parametrized by $\theta \in(\pi, 2 \pi]$ and described as

$$
l(\theta):=\{(r \cos \theta, r \sin \theta): r>0\} .
$$

Obviously, the closure of the half-line $l(\theta)$ in the plane $\mathbb{R}^{2}$ is given by

$$
\overline{l(\theta)}=\{(r \cos \theta, r \sin \theta): r \geq 0\}=l(\theta) \cup\{(0,0)\} .
$$

We note that

$$
H^{+}=\bigcup_{\theta \in(0, \pi]} l(\theta) \text { and } H^{-}=\bigcup_{\theta \in(\pi, 2 \pi]} l(\theta),
$$

and that for each $(\xi, \eta) \in \mathbb{R}^{2} \backslash\{(0,0)\}$, the relation $(\xi, \eta) \in l(\theta)$ determines $\theta \in$ $(0,2 \pi]$ uniquely. We introduce a function $\Theta:(\xi, \eta) \mapsto \theta, \mathbb{R}^{2} \backslash\{(0,0)\} \rightarrow(0,2 \pi]$ by

$$
\Theta(\xi, \eta)= \begin{cases}\cos ^{-1} \frac{\xi}{\sqrt{\xi^{2}+\eta^{2}}} & \text { for }(\xi, \eta) \in H^{+}, \\ \pi+\cos ^{-1} \frac{-\xi}{\sqrt{\xi^{2}+\eta^{2}}}=2 \pi-\cos ^{-1} \frac{\xi}{\sqrt{\xi^{2}+\eta^{2}}} & \text { for }(\xi, \eta) \in H^{-} .\end{cases}
$$


The relation, $(\xi, \eta) \in l(\theta)$, can be stated as $\theta=\Theta(\xi, \eta)$. It is easily seen that the function $\Theta$ is continuous on $\mathbb{R}^{2} \backslash[0, \infty) \times\{0\}$.

Next, let $u \in C^{1}([a, b])$ be a function which satisfies $\left(u(b), u^{\prime}(b)\right) \neq(0,0)$ and observe that

$u(x)>0$ for all $x \in(c, b)$ and some $c \in(a, b)$ if and only if $\left(u^{\prime}(b), u(b)\right) \in H^{+}$,

and

$u(x)<0$ for all $x \in(c, b)$ and some $c \in(a, b)$ if and only if $\left(u^{\prime}(b), u(b)\right) \in H^{-}$.

In view of the direction of outer normal of $[a, b]$ at $a$ or at $b$, for any $u \in C^{1}([a, b])$ we set

$$
B^{-}(u, a):=\left(-u^{\prime}(a), u(a)\right) \in \mathbb{R}^{2} \quad \text { and } \quad B^{+}(u, b):=\left(u^{\prime}(b), u(b)\right) \in \mathbb{R}^{2} .
$$

In the case of $N=1$ we replace the boundary condition (2) by the condition

$$
B^{-}(u, a) \in l\left(\theta^{-}\right) \text {and } B^{+}(u, b) \in l\left(\theta^{+}\right),
$$

with given constants $\theta^{-}, \theta^{+} \in(0,2 \pi]$. This boundary condition for $u \in C^{1}([a, b])$ requires that it should satisfy $\left(u(a), u^{\prime}(a)\right) \neq(0,0)$ and $\left(u(b), u^{\prime}(b)\right) \neq(0,0)$. Later we shall see that if $(\mu, u)$ satisfies (1) and either $\left(u^{\prime}(a), u(a)\right)=(0,0)$ or else $\left(u^{\prime}(b), u(b)\right)=(0,0)$, then $u \equiv 0$ in $[a, b]$ holds. See Proposition 1 . Hence, we may require solutions of (1) to satisfy $\left(u^{\prime}(x), u(x)\right) \neq(0,0)$ at $x=a, b$ without loss of generality. Furthermore, the boundary condition has the positive homogeneity property: if $B^{-}(u, a) \in l\left(\theta^{-}\right)$[resp., $\left.B^{+}(u, b) \in l\left(\theta^{+}\right)\right]$, then $B^{-}(t u, a) \in l\left(\theta^{-}\right)$[resp., $\left.B^{+}(t u, b) \in l\left(\theta^{+}\right)\right]$for all $t>0$. We write $B(u, a, b)=\left(B^{-}(u, a), B^{+}(u, b)\right)$ and $L\left(\theta^{-}, \theta^{+}\right)=l\left(\theta^{-}\right) \times l\left(\theta^{+}\right)$, so that the boundary condition above is stated as $B(u, a, b) \in L\left(\theta^{-}, \theta^{+}\right)$. In the one-dimensional case, our eigenvalue problem is stated as

$$
\begin{aligned}
F\left(u^{\prime \prime}, u^{\prime}, u, x\right)+\mu u & =0 \quad \text { in }(a, b), \\
B(u, a, b) & \in L\left(\theta^{-}, \theta^{+}\right) .
\end{aligned}
$$

The boundary condition (5) prescribes the sign of eigenfunctions near the boundary points $a$ and $b$, and it may be called the unilateral Robin boundary condition.

One of our main interest is the study of sign changing eigenfunctions, and we call an eigenpair $(\mu, u) \in \mathbb{R} \times W^{2,1}(a, b)$ [resp. eigenvalue $\mu$ and eigenfunction $u$ ] of (4) and (5) of $n$th order if $u$ has exactly $n$ zeroes in the interval $(a, b)$.

Finally, we remark that a general (linear) Robin boundary condition for $u \in$ $C^{1}([a, b])$ satisfying $\left(u(x), u^{\prime}(x)\right) \neq(0,0)$, for instance at $a$, is stated as

$$
B^{-}(u, a) \in l(\theta) \cup l(\theta+\pi) \quad \text { for some } \theta \in(0, \pi] .
$$

In the case when $N \geq 2$, we are concerned only with radial eigenpairs of (1) on the ball $B_{R}$. We may identify any radial function $u$ in $B_{R}$ with a function $v$ in $[0, R)$ 
such that $u(x)=v(|x|)$ for a.e. $x \in B_{R}$ and we employ the convention to write $u(x)=u(|x|)$. Similarly to the case $N=1$, in place of the boundary condition (2), we use the condition $B^{+}(u, R) \in l(\theta)$, with a given constant $\theta \in(0,2 \pi]$.

Furthermore, when $N \geq 2$, we assume throughout that $F$ is radially symmetric as stated below. Henceforth $x \otimes x$ denotes the matrix in $\mathbb{S}^{N}$ with the $(i, j)$ entry given by $x_{i} x_{j}$ for $x \in \mathbb{R}^{N}$.

(F4) The function $F$ is radially symmetric, that is, for any $(m, \ell, q, u) \in \mathbb{R}^{4}$ and a.e. $r \in(0, R)$, the function

$$
\omega \mapsto F\left(m \omega \otimes \omega+\ell\left(I_{N}-\omega \otimes \omega\right), q \omega, u, r \omega\right)
$$

is constant on the unit sphere $\mathbf{S}^{N-1}:=\left\{\omega \in \mathbb{R}^{N}:|\omega|=1\right\}$.

We introduce the function $\mathcal{F}: \mathbb{R}^{3} \times(0, R) \rightarrow \mathbb{R}$ by

$$
\mathcal{F}(m, p, u, r):=F\left(m \omega_{0} \otimes \omega_{0}+(p / r)\left(I_{N}-\omega_{0} \otimes \omega_{0}\right), p \omega_{0}, u, r \omega_{0}\right),
$$

where $\omega_{0}$ is any fixed unit vector in $\mathbf{S}^{N-1}$. We remark $([21,25])$ that if $u(x)=v(|x|)$ and $v \in W_{\text {loc }}^{2,1}(0, R)$, then for a.e. $r \in(0, R)$ and for all $\omega \in \mathbf{S}^{N-1}$, (F4) implies

$$
F\left(D^{2} u(r \omega), D u(r \omega), u(r \omega), r \omega\right)=\mathcal{F}\left(v^{\prime \prime}(r), v^{\prime}(r), v(r), r\right) .
$$

We warn the reader that the definition of $\mathcal{F}$ (also $\mathcal{P}^{ \pm}$which will appear later) is not the same as in [25].

Next, for $N \geq 2$ and $q \in[1, \infty]$, let $W_{\mathrm{r}}^{2, q}\left(B_{R}\right)$ denote the space of those functions in $W^{2, q}\left(B_{R}\right)$ which are radially symmetric. This space is also denoted by $W_{\mathrm{r}}^{2, q}(0, R)$ when all functions $u \in W_{\mathrm{r}}^{2, q}\left(B_{R}\right)$ are regarded as functions on $(0, R)$. Similarly, we write $L_{\mathrm{r}}^{q}(0, R)$ for the space of all radial functions in $L^{q}\left(B_{R}\right)$. According to the Sobolev embedding theorem, if $q>N / 2$, then we may regard $u \in W_{\mathrm{r}}^{2, q}(0, R)$ as a function in $C([0, R]) \cap C^{1}((0, R])$. Thus, according to (6), the eigenvalue problem in multi-dimensional case may be stated for $u \in W_{\mathrm{r}}^{2, q}(0, R)$ as

$$
\begin{aligned}
\mathcal{F}\left(u^{\prime \prime}, u^{\prime}, u, r\right)+\mu u & =0 \quad \text { a.e. in }(0, R), \\
B^{+}(u, R) & \in l(\theta) .
\end{aligned}
$$

Any radial eigenpair $(\mu, u)$ of $(1)$, when $u$ is regarded as a function on $[0, R]$, is simply an eigenpair of (7). We call an eigenpair $(\mu, u)$ [resp. an eigenvalue $\mu$ and an eigenfunction $u]$ of (7) and (8) of $n$th order if $u$ has exactly $n$ zeroes in $[0, R)$ as a function on $[0, R]$. We note that a radial eigenpair $(\mu, u)$ is a principal eigenpair if and only if it is of zeroth order as an eigenpair of (7)-(8).

The main contributions in this paper are described briefly as follows.

We show the existence of $n$th order eigenpairs of (4)-(5) and of (7)-(8) for any $n \in \mathbb{N} \cup\{0\}$, provided the triplet $\left(n, \theta^{-}, \theta^{+}\right)$is admissible when $N=1$ [see for the admissibility the second paragraph after Proposition 1 below]. This is done under the same hypotheses on $F$ as those in [25], where eigenvalue problems with the Dirichlet 
boundary condition are treated, except for the case $(N, \Lambda)=(1, \infty)$. Furthermore, our requirement on the exponent $q$ from (F2) seems relatively sharp, as remarked in [25], in the viewpoint of the existence of eigenfunctions or solutions of (1). For comparison, we refer to [15, 16,20,23,27,29,32].

In this paper, to establish the existence of eigenpairs, we employ the shooting method in ODE theory, while the so-called inverse power method is adapted in [25].

We establish general comparison theorems for $n$th order eigenvalues with possibly different $n$ 's and angles $\left(\theta^{-}, \theta^{+}\right)$(or $\theta$ in the case of $N \geq 2$ ).

It may be a general principle (cf. Berestycki, Nirenberg and Varadhan [5] and Lions [28]) that the solvability of the inhomogeneous PDE

$$
F\left(D^{2} u, D u, u, x\right)+\mu u+f(x)=0 \text { in } \Omega,
$$

where $f \in L^{q}(\Omega)$ is a given nonnegative function, with the boundary condition like (5) or (8) is closely related to the principal eigenvalues. We establish general theorems in this direction that relate the solvability of boundary value problems for the inhomogeneous PDE

$$
F\left(D^{2} u, D u, u, x\right)+\mu u+\operatorname{sgn}(u) f(x)=0 \quad \text { in } \Omega
$$

and the $n$th order eigenvalue for the corresponding homogeneous PDE.

It is also a general understanding (cf. $[5,28]$ ) that the principal eigenvalues are thresholds to the validity of the maximum principle for PDE (1). See also [1,3,7$10,12,13,24,26,31,32]$. Theorems 16 and 34 below state roughly that the principal eigenvalues have this role of threshold for our general boundary value problems. See also the comments after Theorem 16.

Two other characterizations of $n$th order eigenvalues are formulated and established via the existence of $n$th order solutions (i.e. solutions having $n$ zeroes) of differential inequalities [see (16), (17), (24) and (25)].

This paper is organized as follows. We present the main results in the onedimensional and radial cases in Sects. 2 and 3, respectively. Sections 4 and 6 provide preliminary observations, including both the strong and weak maximum principles, needed for the proofs of the mains results in the one-dimensional and radial cases, respectively. The proofs of the main results are provided in Sects. 5 and 7. In Sect. 8, we give two examples that have many first order solutions of (9).

Notation We denote by $\mathbb{N}_{0}$ the set of all nonnegative integers, that is, $\mathbb{N}_{0}=\mathbb{N} \cup\{0\}$. Given a function $f$ on $\Omega$ which may not be continuous, we write $f=0$ in $\Omega$ for writing $f=0$ a.e. in $\Omega, f>0$ in $\Omega$ for writing $f>0$ a.e. in $\Omega$, etc. We regard $u \in W^{2, q}(a, b)$, with $q \in[1, \infty]$, as a $C^{1}$-function on $[a, b]$ in view of the Sobolev embedding theorem. We use the notation $F[u]$ to denote the function $x \mapsto$ $F\left(u^{\prime \prime}(x), u^{\prime}(x), u(x), x\right)$. The sign function is denoted by sgn, that is, sgn $: \mathbb{R} \rightarrow \mathbb{R}$ is the function defined by $\operatorname{sgn}(r)=1$ for $r>0, \operatorname{sgn}(0)=0$ and $\operatorname{sgn}(r)=-1$ for $r<0$.

\section{Main results in the one-dimensional case}

In this section we are concerned with the one-dimensional case and present the main results concerning the eigenvalue problem (4)-(5). It is worth emphasizing at this point 
that the boundary condition (5) has the positive homogeneity of degree one in the sense that if $u \in C^{1}([a, b])$ satisfies (5), then so does the function $t u$ for any $t>0$. A similar remark applies to the boundary condition (8). The proofs of the results in this section are given in Sect. 5 .

We begin with a few basic observations on solutions of (4).

Proposition 1 Assume that (F1) and (F2) hold and that the function $F(0,0,0, x) \equiv 0$ in $(a, b)$. Let $(\mu, \varphi) \in \mathbb{R} \times W^{2,1}(a, b)$ be such that $u$ is a solution of (4), with the given $\mu$, and $\varphi(x) \not \equiv 0$ on $[a, b]$. Then

(1) $\left(\varphi(x), \varphi^{\prime}(x)\right) \neq(0,0)$ for all $x \in[a, b]$.

(2) The function $\varphi$ has a finite number of zeroes in $[a, b]$.

(3) The function $\varphi$ changes sign at every zeroes of $\varphi$ in $(a, b)$.

(4) Let $n$ be the number of zeroes of $\varphi$ in $(a, b)$ and let $\theta^{-}, \theta^{+} \in(0,2 \pi]$ be such that $B^{-}(\varphi, a) \in l\left(\theta^{-}\right)$and $B^{+}(\varphi, b) \in l\left(\theta^{+}\right)$. If $n$ is an even integer, then we have either $\theta^{-}, \theta^{+} \in(0, \pi]$ or $\theta^{-}, \theta^{+} \in(\pi, 2 \pi]$. Otherwise, we have either $\theta^{-}, \theta^{+}-\pi \in(0, \pi]$ or $\theta^{-}-\pi, \theta^{+} \in(0, \pi]$.

We introduce an "indicator" function $i:(0,2 \pi] \rightarrow\{0,1\}$ by setting $i(t)=0$ if $t \in(0, \pi]$ and $i(t)=1$ otherwise.

Let $\left(n, \theta^{-}, \theta^{+}\right) \in \mathbb{N}_{0} \times(0,2 \pi]^{2}$. We say that $\left(n, \theta^{-}, \theta^{+}\right)$is admissible if either $n$ is even and $i\left(\theta^{-}\right)=i\left(\theta^{+}\right)$or $n$ is odd and $i\left(\theta^{-}\right) \neq i\left(\theta^{+}\right)$.

One of our main results for $N=1$ is stated as follows.

Theorem 2 Let $\left(n, \theta^{-}, \theta^{+}\right) \in \mathbb{N}_{0} \times(0,2 \pi]^{2}$. Assume that (F1)-(F3) hold and $\left(n, \theta^{-}, \theta^{+}\right)$is admissible. (1) For $i=1,2$, let $\left(\mu_{i}, \varphi_{i}\right) \in \mathbb{R} \times W^{2,1}(a, b)$ be an nth order eigenpair of (4)-(5). Then $\mu_{1}=\mu_{2}$ and, if, in addition, $\left\|\varphi_{1}\right\|_{L^{\infty}(a, b)}=$ $\left\|\varphi_{2}\right\|_{L^{\infty}(a, b)}$, then $\varphi_{1}=\varphi_{2}$ on $[a, b]$. (2) There exists an nth order eigenpair $(\mu, \varphi) \in \mathbb{R} \times W^{2, q}(a, b)$ of (4)-(5).

It follows from the theorem above that an $n$th order eigenvalue of (4)-(5) exists and is unique. Henceforth, we use the notation $\mu^{n}\left(\theta^{-}, \theta^{+}, a, b\right)$ to denote a unique $n$th order eigenvalue of (4)-(5) for any admissible $\left(n, \theta^{-}, \theta^{+}\right) \in \mathbb{N}_{0} \times(0,2 \pi]^{2}$.

According to Proposition 1 and Theorem 2, the admissibility of $\left(n, \theta^{-}, \theta^{+}\right) \in$ $\mathbb{N}_{0} \times(0,2 \pi]^{2}$ is a necessary and sufficient condition for the existence of $n$th order eigenpairs of (4)-(5).

If we replace the unilateral boundary condition (5) by a bilateral boundary condition [see (10) below], then the notion of admissibility does not make sense. Indeed, let $\theta_{1}^{-}, \theta_{1}^{+} \in(0, \pi]$ and $\theta_{2}^{-}, \theta_{2}^{+} \in(\pi, 2 \pi]$, and consider the boundary condition

$$
B^{-}(u, a) \in l\left(\theta_{1}^{-}\right) \cup l\left(\theta_{2}^{-}\right) \quad \text { and } \quad B^{+}(u, b) \in l\left(\theta_{1}^{+}\right) \cup l\left(\theta_{2}^{+}\right) .
$$

It is easily seen that for any $n \in \mathbb{N}_{0}$, either $\left(n, \theta_{1}^{-}, \theta_{1}^{+}\right)$or $\left(n, \theta_{1}^{-}, \theta_{2}^{+}\right)$is admissible. Similarly, either $\left(n, \theta_{2}^{-}, \theta_{1}^{+}\right)$or $\left(n, \theta_{2}^{-}, \theta_{2}^{+}\right)$is admissible.

Note that the boundary condition $(10)$ in the case where $\left(\theta_{1}^{-}, \theta_{2}^{-}\right)=(\pi, 2 \pi)$ [resp. the case where $\left.\left(\theta_{1}^{+}, \theta_{2}^{+}\right)=(\pi, 2 \pi)\right]$ corresponds to the Dirichlet condition at $a$ [resp. at $b$ ], (10) in the case where $\left(\theta_{1}^{-}, \theta_{2}^{-}\right)=(\pi / 2,3 \pi / 2)$ [resp. the case where $\left.\left(\theta_{1}^{+}, \theta_{2}^{+}\right)=(\pi / 2,3 \pi / 2)\right]$ corresponds to the Neumann condition at $a$ [resp. at $b$ ], 
and (10) in the case where $\theta_{1}^{-} \in(0, \pi)$ and $\theta_{2}^{-}=\pi+\theta_{1}^{-}$corresponds to [resp. the case where $\theta_{1}^{+} \in(0, \pi)$ and $\left.\theta_{2}^{+}=\pi+\theta_{1}^{+}\right]$a general linear Robin condition at $a$ [resp. at $b$ ].

The following corollary generalizes [25, Theorem 1.1] to the general first-order boundary condition.

Corollary 3 Assume (F1)-(F3) hold. Let $n \in \mathbb{N}_{0}, \theta_{1}^{-}, \theta_{1}^{+} \in(0, \pi]$ and $\theta_{2}^{-}, \theta_{2}^{+} \in$ $(\pi, 2 \pi]$. Then there exist nth order eigenpairs $\left\{\left(\mu^{ \pm}, \varphi^{ \pm}\right)\right\} \subset \mathbb{R} \times W^{2, q}(a, b)$ of (4) and (10) and increasing sequences $\left\{x_{k}^{ \pm}\right\}_{k=0}^{n+1} \subset[a, b]$, with $x_{0}^{ \pm}=a$ and $x_{n+1}^{ \pm}=b$, such that

(1) $\left\|\varphi^{ \pm}\right\|_{L^{\infty}(a, b)}=1$ and $\pm(-1)^{i-1} \varphi^{ \pm}>0$ in $\left(x_{i-1}^{ \pm}, x_{i}^{ \pm}\right)$for all $1 \leq i \leq n+1$.

(2) If $(\mu, \varphi) \in \mathbb{R} \times W^{2, q}(a, b)$ is an nth order eigenpair of (4) and (10) with $\|\varphi\|_{L^{\infty}(a, b)}=1$, then either $(\mu, \varphi)=\left(\mu^{+}, \varphi^{+}\right)$or else $(\mu, \varphi)=\left(\mu^{-}, \varphi^{-}\right)$.

An important remark complementing Corollary 3 is that, due to Proposition 1, every eigenfunction of (4) and (10) has at most a finite number of zeroes.

To state a general comparison theorem of eigenvalues, we introduce a partial order relation $\leq$ on the set of all admissible $(n, \theta) \in \mathbb{N}_{0} \times(0,2 \pi]^{2}$. Let $\alpha_{1}:=$ $\left(n_{1}, \theta_{1}\right), \alpha_{2}:=\left(n_{2}, \theta_{2}\right) \in \mathbb{N}_{0} \times(0,2 \pi]^{2}$ be admissible. We write $\alpha_{1} \leq \alpha_{2}$ if there exist $k^{-}, k^{+} \in \mathbb{N}_{0}$ having the properties (11)-(15) below:

$$
\begin{aligned}
& n_{1}+k^{-}+k^{+}=n_{2} \\
& i\left(\theta_{1}^{-}\right)+k^{-} \equiv i\left(\theta_{2}^{-}\right) \quad(\bmod 2) \\
& i\left(\theta_{1}^{+}\right)+k^{+} \equiv i\left(\theta_{2}^{+}\right) \quad(\bmod 2) \\
& \theta_{1}^{-} \leq \theta_{2}^{-} \quad \text { if } k^{-}=0 \\
& \theta_{1}^{+} \leq \theta_{2}^{+} \quad \text { if } k^{+}=0
\end{aligned}
$$

where $\theta_{i}=\left(\theta_{i}^{-}, \theta_{i}^{+}\right) \in(0,2 \pi] \times(0,2 \pi]$ for $i=1,2$.

As above, let $\alpha_{1}:=\left(n_{1}, \theta_{1}\right), \alpha_{2}:=\left(n_{2}, \theta_{2}\right) \in \mathbb{N}_{0} \times(0,2 \pi]^{2}$ be admissible. It is easily checked that, in the case when $n_{1}=n_{2}$, we have $\alpha_{1} \leq \alpha_{2}$ if and only if $i\left(\theta_{1}^{-}\right)=i\left(\theta_{2}^{-}\right), i\left(\theta_{1}^{+}\right)=i\left(\theta_{2}^{+}\right), \theta_{1}^{-} \leq \theta_{2}^{-}$and $\theta_{1}^{+} \leq \theta_{2}^{+}$. Note moreover that when $n_{1}=n_{2}$, we have $i\left(\theta_{1}^{-}\right)=i\left(\theta_{2}^{-}\right)$and $i\left(\theta_{1}^{+}\right)=i\left(\theta_{2}^{+}\right)$if and only if either $i\left(\theta_{1}^{-}\right)=i\left(\theta_{2}^{-}\right)$or $i\left(\theta_{1}^{+}\right)=i\left(\theta_{2}^{+}\right)$. Also, when $n_{1}+1=n_{2}$, we have $\alpha_{1} \leq \alpha_{2}$ if and only if either $i\left(\theta_{1}^{-}\right)=i\left(\theta_{2}^{-}\right)$and $\theta_{1}^{-} \leq \theta_{2}^{-}$or $i\left(\theta_{1}^{+}\right)=i\left(\theta_{2}^{+}\right)$and $\theta_{1}^{+} \leq \theta_{2}^{+}$. When $n_{1}+2=n_{2}$, we have $\alpha_{1} \leq \alpha_{2}$ if and only if one of the following three conditions holds: (1) $i\left(\theta_{1}^{-}\right)=i\left(\theta_{2}^{-}\right)$and $\theta_{1}^{-} \leq \theta_{2}^{-}$, (2) $i\left(\theta_{1}^{+}\right)=i\left(\theta_{2}^{+}\right)$and $\theta_{1}^{+} \leq \theta_{2}^{+}$and (3) $i\left(\theta_{1}^{-}\right) \neq i\left(\theta_{2}^{-}\right)$. This last condition is equivalent to the condition, $i\left(\theta_{1}^{+}\right) \neq i\left(\theta_{2}^{+}\right)$. Furthermore, when $n_{1}+3 \leq n_{2}$, we have always $\alpha_{1} \leq \alpha_{2}$.

Theorem 4 Assume that (F1)-(F3) hold. Let $\left(n_{1}, \theta_{1}\right),\left(n_{2}, \theta_{2}\right) \in \mathbb{N}_{0} \times(0,2 \pi]^{2}$ be admissible. If $\left(n_{1}, \theta_{1}\right) \leq\left(n_{2}, \theta_{2}\right)$, then $\mu^{n_{1}}\left(\theta_{1}, a, b\right) \leq \mu^{n_{2}}\left(\theta_{2}, a, b\right)$.

We consider the differential inequalities

$$
(F[u]+\mu u) u \leq 0 \quad \text { in }(a, b),
$$


and

$$
(F[u]+\mu u) u \geq 0 \quad \text { in }(a, b),
$$

and we give characterizations of the $n$th order eigenvalues based on solutions $(\mu, u)$ of (16) or (17).

Let $(\mu, u) \in \mathbb{R} \times W^{2,1}(a, b)$. We call such a pair $(\mu, u)$ a solution of (16) [resp. (17)] and (5) if it satisfies (16) [resp. (17)] and (5), and call it an $n$th order solution of (16) [resp. (17)] and (5) if $u$ has exactly $n$ zeroes in $(a, b)$ and changes sign at each zero of $u$ in $(a, b)$.

We denote by $E^{-}\left(n, \theta^{-}, \theta^{+}\right)$[resp. $\left.E^{+}\left(n, \theta^{-}, \theta^{+}\right)\right]$the set of $\mu \in \mathbb{R}$ such that, for some $u \in W^{2,1}(a, b)$, the pair $(\mu, u)$ is an $n$th order solution of (16) [resp. (17)] and (5). We set

$$
\begin{aligned}
& \widetilde{E}^{-}(n, \theta)=\bigcup\left\{E^{-}(\alpha): \alpha \text { admissible, } \alpha \leq(n, \theta)\right\}, \\
& \widetilde{E}^{+}(n, \theta)=\bigcup\left\{E^{+}(\alpha): \alpha \text { admissible, } \alpha \geq(n, \theta)\right\} .
\end{aligned}
$$

Theorem 5 Under the hypotheses $(\mathrm{F} 1)-(\mathrm{F} 3)$, if $(n, \theta) \in \mathbb{N}_{0} \times(0,2 \pi]^{2}$ is admissible, then

$$
\mu^{n}(\theta, a, b)=\max E^{-}(n, \theta)=\max \widetilde{E}^{-}(n, \theta)=\min E^{+}(n, \theta)=\min \widetilde{E}^{+}(n, \theta) .
$$

Given $\mu \in \mathbb{R}, f \in L^{1}(a, b)$ and $\theta \in(0,2 \pi]^{2}$, we consider the solvability of the boundary value problem for the inhomogeneous ODE

$$
F[u]+\mu u+\operatorname{sgn}(u) f=0 \text { in }(a, b) \text { and } B(u, a, b) \in L(\theta) .
$$

We say that $u$ is an $n$th order solution of (18) if $u$ satisfies (18), has exactly $n$ zeroes in $(a, b)$ and changes sign at each zero. Regarding the solvability of (18), we have the following result.

Theorem 6 Assume that (F1)-(F3) hold. Let $f \in L^{1}(a, b), n \in \mathbb{N}_{0}, \theta \in(0,2 \pi]^{2}$, and $\mu \in \mathbb{R}$. Assume that $f \geq 0$ and $f \neq \equiv$ in $(a, b)$ and $(n, \theta)$ is admissible. (1) If $\mu<\mu^{n}(\theta, a, b)$, then there exists an nth order solution of (18). (2) If $\mu<$ $\mu^{0}(\theta, a, b)$, then the zeroth order solution of (18) is unique. (3) If $\mu \geq \mu^{n}(\theta, a, b)$, then there exists no nth order solution of (18).

The uniqueness of first order solutions of (18) does not hold in general as is shown in Sect. 8.

\section{Main results in the radial case}

In this section, we assume throughout that $N \geq 2$ and $\Omega=B_{R}$ and we deal with the ODE (7).

As in [25], under the assumption that $\Lambda<\infty$, we set $\lambda_{*}=\lambda / \Lambda$ and $q_{*}=$ $N /\left(\lambda_{*} N+1-\lambda_{*}\right)$. Note that $0<\lambda_{*} \leq 1$ and $q_{*} \in[1, N)$. 
In the radial case, we need to specify the integrability of functions given in (F2).

(F5) For the exponent $q$ from (F2), the inequality $q>\max \left\{N / 2, q_{*}\right\}$ holds and $\beta \in L^{N}\left(B_{R}\right)$ if $q<N$.

Theorem 7 Assume that (F1)-(F5) hold. Let $(n, \theta) \in \mathbb{N}_{0} \times(0,2 \pi]$. (1) For $i=1,2$, let $\left(\mu_{i}, \varphi_{i}\right) \in \mathbb{R} \times W_{\mathrm{r}}^{2, q}(0, R)$ be an $n$th order eigenpair of (7)-(8). Then $\mu_{1}=\mu_{2}$ and if, in addition, $\left\|\varphi_{1}\right\|_{L^{\infty}(0, R)}=\left\|\varphi_{2}\right\|_{L^{\infty}(0, R)}$, then $\varphi_{1}=\varphi_{2}$ on $[0, R]$. (2) There exists an nth order eigenpair $(\mu, \varphi) \in \mathbb{R} \times W_{\mathrm{r}}^{2, q}(0, R)$ of (7)-(8).

In view of the unique existence of an $n$th order eigenvalue of (7)-(8) due to the theorem above, we may denote by $\mu_{\mathrm{r}}^{n}(\theta, R)$ the $n$th order eigenvalue of (7) and (8).

Let $\left(\theta_{1}, \theta_{2}\right) \in(0, \pi] \times(\pi, 2 \pi]$, and we introduce a bilateral version of the boundary condition (8) as follows.

$$
B^{+}(u, R) \in l\left(\theta_{1}\right) \cup l\left(\theta_{2}\right) .
$$

Corollary 8 Assume that (F1)-(F5) hold. Let $\left(n, \theta_{1}, \theta_{2}\right) \in \mathbb{N}_{0} \times(0, \pi] \times(\pi, 2 \pi]$. Then there exist nth order eigenpairs $\left\{\left(\mu^{ \pm}, \varphi^{ \pm}\right)\right\} \subset \mathbb{R} \times W_{\mathrm{r}}^{2, q}(0, R)$ of (7) and (19), and increasing sequences $\left\{r_{i}^{ \pm}\right\}_{i=0}^{n+1} \subset[0, R]$, with $r_{0}^{ \pm}=0$ and $r_{n+1}^{ \pm}=R$, such that

(1) $\left\|\varphi^{ \pm}\right\|_{L^{\infty}(0, R)}=1, \pm \varphi^{ \pm}(0)>0$ and $\pm(-1)^{i-1} \varphi^{ \pm}>0$ in $\left(r_{i-1}, r_{i}\right)$ for every $1 \leq i \leq n+1$.

(2) If $(\mu, \varphi) \in \mathbb{R} \times W_{\mathrm{r}}^{2, q}(0, R)$ is an nth order eigenpair of (7)-(19) with $\|\varphi\|_{L^{\infty}(0, R)}=1$, then either $(\mu, \varphi)=\left(\mu^{+}, \varphi^{+}\right)$or $(\mu, \varphi)=\left(\mu^{-}, \varphi^{-}\right)$.

We state the following theorem which is a counterpart of Theorem 4 in the onedimensional case.

For $\alpha_{1}=\left(n_{1}, \theta_{1}\right), \alpha_{2}=\left(n_{2}, \theta_{2}\right) \in \mathbb{N}_{0} \times[0,2 \pi)$, we write $\alpha_{1} \leq \alpha_{2}$ if one of the following four conditions holds.

$$
\begin{aligned}
& n_{2} \geq n_{1}+2, \\
& n_{2}=n_{1}+1, \quad i\left(\theta_{1}\right) \neq i\left(\theta_{2}\right), \\
& n_{2}=n_{1}+1, \quad i\left(\theta_{1}\right)=i\left(\theta_{2}\right), \quad \theta_{1} \leq \theta_{2} \\
& n_{2}=n_{1}, \quad i\left(\theta_{1}\right)=i\left(\theta_{2}\right), \quad \theta_{1} \leq \theta_{2} .
\end{aligned}
$$

Theorem 9 Assume that (F1)-(F5) hold. Let $\left(n_{1}, \theta_{1}\right),\left(n_{2}, \theta_{2}\right) \in \mathbb{N}_{0} \times(0,2 \pi]$. If $\left(n_{1}, \theta_{1}\right) \leq\left(n_{2}, \theta_{2}\right)$, then $\mu_{\mathrm{r}}^{n_{1}}\left(\theta_{1}, R\right) \leq \mu_{\mathrm{r}}^{n_{2}}\left(\theta_{2}, R\right)$.

Next, we proceed to give characterizations by $n$th order solutions to the following inequalities as in Sect. 2:

$$
\begin{array}{ll}
(F[u]+\mu u) u \leq 0 & \text { in } B_{R}, \\
(F[u]+\mu u) u \geq 0 & \text { in } B_{R} .
\end{array}
$$

Here we call $u \in W_{\mathrm{r}}^{2, q}(0, R)$ an $n$th order solution of (24) [resp. (25)] and (8) if $u$ satisfies (24) [resp. (25)] and (8), has exactly $n$ zeroes in $(0, R)$ and changes sign at each zero of it in $(0, R)$. 
For any $\alpha=(n, \theta) \in \mathbb{N}_{0} \times(0,2 \pi]$, we denote by $E_{\mathrm{r}}^{-}(\alpha)\left[\right.$ resp. $\left.E_{\mathrm{r}}^{+}(\alpha)\right]$ the set of all $\mu \in \mathbb{R}$ for which there corresponds a function $u \in W_{\mathrm{r}}^{2, q}(0, R)$ such that $(\mu, u)$ is an $n$th order solution of (24) [resp. (25)] and (8). We set

$$
\begin{aligned}
& \widetilde{E}_{\mathrm{r}}^{-}(\alpha):=\bigcup\left\{E_{\mathrm{r}}^{-}(\tilde{\alpha}): \tilde{\alpha} \in \mathbb{N}_{0} \times(0,2 \pi], \tilde{\alpha} \leq \alpha\right\}, \\
& \widetilde{E}_{\mathrm{r}}^{+}(\alpha):=\bigcup\left\{E_{\mathrm{r}}^{+}(\tilde{\alpha}): \tilde{\alpha} \in \mathbb{N}_{0} \times(0,2 \pi], \tilde{\alpha} \geq \alpha\right\} .
\end{aligned}
$$

Theorem 10 Under the hypotheses (F1)-(F5), for every $\alpha=(n, \theta) \in \mathbb{N}_{0} \times(0,2 \pi]$ we have

$$
\mu_{\mathrm{r}}^{n}(\theta, R)=\max E_{\mathrm{r}}^{-}(\alpha)=\max \widetilde{E}_{\mathrm{r}}^{-}(\alpha)=\min E_{\mathrm{r}}^{+}(\alpha)=\min \widetilde{E}_{\mathrm{r}}^{+}(\alpha) .
$$

Finally, we consider the solvability of the boundary value problem

$$
F[u]+\mu u+\operatorname{sgn}(u) f=0 \text { in } B_{R} \quad \text { and } \quad B^{+}(u, R) \in l(\theta),
$$

where $\theta \in(0,2 \pi], \mu \in \mathbb{R}$ and $f \in L_{\mathrm{r}}^{q}(0, R)$. A function $u \in W_{\mathrm{r}}^{2, q}(0, R)$ is called an $n$th order solution of (26) if it satisfies (26), has exactly $n$ zeroes in $(0, R)$ and changes sign at every zeroes of it in $(0, R)$.

Theorem 11 Assume that (F1)-(F5) hold. Let $(n, \theta) \in \mathbb{N}_{0} \times(0,2 \pi], \mu \in \mathbb{R}$ and $f \in L_{\mathrm{r}}^{q}(0, R)$. Assume that $f \geq 0$ and $f \not \equiv 0$ in $(0, R)$. (1) If $\mu<\mu_{\mathrm{r}}^{n}(\theta, R)$, then there exists an nth order solution of (26). (2) If $\mu<\mu_{\mathrm{r}}^{0}(\theta, R)$, then the zeroth order solution of (26) is unique. (3) If $\mu \geq \mu_{\mathrm{r}}^{n}(\theta, R)$, then there exists no nth order solution of (26).

When $n=1$, the $n$th order solution of (26) in the claim (1) of the theorem above is not unique in general. An example that shows this failure of uniqueness is given in Sect. 8.

\section{Preliminary observations and results in the one-dimensional case}

This section deals with the case $N=1$ and discusses some basic observations and results concerning (4).

\subsection{Two basic symmetries}

We state two structural symmetries of eigenvalue problem (4)-(5) under reflection, which will be useful for simplification of our presentation.

(S1) Let $(\mu, u) \in \mathbb{R} \times W^{2,1}(a, b), F: \mathbb{R}^{3} \times(a, b) \rightarrow \mathbb{R}$ and $\left(\theta^{-}, \theta^{+}\right) \in(0,2 \pi]^{2}$. Set $F^{-}(m, p, r, x):=-F(-m,-p,-r, x)$ for $(m, p, r, x) \in \mathbb{R}^{3} \times(a, b)$ and $u^{-}(x):=-u(x)$ for $x \in[a, b]$, and select $\left(\theta_{1}^{-}, \theta_{1}^{+}\right) \in(0,2 \pi]^{2}$ so that $\theta_{1}^{-} \equiv \theta^{-}-\pi, \theta_{1}^{+} \equiv \theta^{+}-\pi \quad(\bmod 2 \pi)$. If $(\mu, u)$ satisfies $(4)-(5)$, then 


$$
\left\{\begin{array}{l}
F^{-}\left[u^{-}\right]+\mu u^{-}=0 \quad \text { a.e. in }(a, b), \\
B^{-}\left(u^{-}, a\right) \in l\left(\theta_{1}^{-}\right) \text {and } B^{+}\left(u^{-}, b\right) \in l\left(\theta_{1}^{+}\right)
\end{array}\right.
$$

and the converse is also true.

This is a simple observation and is easily checked. Notice that the dualities $\left(F^{-}\right)^{-}=$ $F$ and $\left(u^{-}\right)^{-}=u$ hold. We note moreover that if $F$ satisfies (F1)-(F3), then so does $F^{-}$.

(S2) Let $(\mu, u) \in \mathbb{R} \times W^{2,1}(a, b), F: \mathbb{R}^{3} \times(a, b) \rightarrow \mathbb{R}$ and $\left(\theta^{-}, \theta^{+}\right) \in(0,2 \pi]^{2}$. Set $F^{\sim}(m, p, r, x):=F(m,-p, r,-x)$ for $(m, p, r, x) \in \mathbb{R}^{3} \times(-b,-a)$ and $u^{\sim}(x):=u(-x)$ for $x \in[-b,-a]$. If $(\mu, u)$ satisfies (4)-(5), then

$$
\left\{\begin{array}{l}
F^{\sim}\left[u^{\sim}\right]+\mu u^{\sim}=0 \text { a.e. in }(-b,-a) \\
B^{-}\left(u^{\tilde{u}},-b\right) \in l\left(\theta^{+}\right) \text {and } B^{+}\left(u^{\tilde{}},-a\right) \in l\left(\theta^{-}\right)
\end{array}\right.
$$

and the converse is also true.

The proof of this property is straightforward by observing that the dualities $\left(F^{\sim}\right)^{\sim}=F$ and $\left(u^{\sim}\right)^{\sim}=u$ hold and that $B^{-}\left(u^{\sim},-b\right)=\left(-\left(u^{\sim}\right)^{\prime}(-b), u^{\sim}(-b)\right)=$ $\left(u^{\prime}(b), u(b)\right)=B^{+}(u, b)$ and $B^{+}\left(u^{\sim},-a\right)=\left(\left(u^{\sim}\right)^{\prime}(-a), u^{\sim}(-a)\right)=\left(-u^{\prime}(a), u(a)\right)$ $=B^{-}(u, a)$. Furthermore, we remark that conditions (F1)-(F3) hold for $F^{\sim}$.

\subsection{Proof of Proposition 1}

We give here a proof of Proposition 1, for which we need a result from [25] [see also [21]].

Lemma 12 Let $F$ satisfy (F1) and (F2). Then there exists a Carathéodry function $g_{F}: \mathbb{R}^{3} \times(a, b) \rightarrow \mathbb{R}$ such that for a.e. $x \in(a, b)$ and any $(m, p, u, d) \in \mathbb{R}^{4}, m=$ $g_{F}(p, u, d, x)$ holds if and only if $F(m, p, u, x)=d$. Moreover, the estimates

$$
\begin{aligned}
\left|g_{F}(0,0,0, x)\right| \leq & \lambda^{-1}|F[0](x)| \\
\left|g_{F}\left(p_{1}, u_{1}, d_{1}, x\right)-g_{F}\left(p_{2}, u_{2}, d_{2}, x\right)\right| \leq & \lambda^{-1}\left(\beta(x)\left|p_{1}-p_{2}\right|+\gamma(x)\left|u_{1}-u_{2}\right|\right. \\
& \left.+\left|d_{1}-d_{2}\right|\right)
\end{aligned}
$$

for all $(p, u, d),\left(p_{1}, u_{1}, d_{1}\right),\left(p_{2}, u_{2}, d_{2}\right) \in \mathbb{R}^{3}$ and a.e. $x \in(a, b)$.

This lemma is a consequence of [25, Lemma 2.1], except that the function $g_{F}$ is a Carathéodry function on $\mathbb{R}^{3} \times(a, b)$. Arguing as in [21,25], for each $m, p, u, d \in \mathbb{R}^{4}$, we find that for any $(m, p, u) \in \mathbb{R}^{3}$,

$$
\left\{x \in(a, b): m \leq g_{F}(p, u, d, x)\right\}=\{x \in(a, b): F(m, p, u, x) \leq d\},
$$

which says that the function $x \mapsto g_{F}(p, u, d, x)$ is measurable for every $(p, u, d) \in$ $\mathbb{R}^{3}$. The continuity of $g_{F}(m, p, u, x)$ in $(p, u, d)$ follows from the second inequality in Lemma 12. 
The lemma above allows us to rewrite (4) in the normal form

$$
u^{\prime \prime}(x)=g_{F}\left(u^{\prime}, u,-\mu u, x\right) \text { in }(a, b) .
$$

This observation and the general theory of ODE assure that, given $c \in[a, b]$ and $(p, q) \in \mathbb{R}^{2}$, under the assumptions that (F1) and (F2) hold, the initial value problem

$$
F[u]=0 \quad \text { in }(a, b) \quad \text { and } \quad\left(u(c), u^{\prime}(c)\right)=(p, q)
$$

has a unique solution $u \in W^{2, q}(a, b)$.

Let $c, d \in[a, b]$ be such that $a \leq c<d \leq b$. Under the assumptions (F1) and (F2) hold, given a solution $u \in W^{2,1}(c, d)$ of (4), by solving the initial value problem (28) in $[a, b]$, with $(p, q)=\left(u(c), u^{\prime}(c)\right)$, we can always extend the domain of definition of $u$ to $[a, b]$ as a solution of (4). In what follows, under the assumptions of (F1) and (F2), we may and do regard a solution $u \in W^{2,1}(c, d)$ of (4) in $(c, d)$, with $a \leq c<d \leq b$, as a function in $W^{2,1}(a, b)$ that is a solution of (4) in $(a, b)$.

Proof of Proposition 1 By assumption, we have $F[0] \equiv 0$ in $(a, b)$ and, hence, $u(x) \equiv 0$ is a solution of (4). We see by the uniqueness of solutions of the initial value problem (28) that if $\left(\varphi(c), \varphi^{\prime}(c)\right)=(0,0)$ for some $c \in[a, b]$, then $\varphi(x) \equiv 0$ in $[a, b]$. Claim (1) follows readily from this observation.

To see that every zero of the function $\varphi$ is isolated, we assume that $\varphi(c)=0$ at some $c \in[a, b]$, which implies that $\varphi^{\prime}(c) \neq 0$, and observe that $\varphi$ is increasing or decreasing near the point $c$. All the zeroes of $\varphi$ are thus isolated points in $[a, b]$ and hence the number of the zeroes is finite. That is, claim (2) is valid.

Let $c \in(a, b)$ be a zero of $\varphi$. Then the function $\varphi$ is increasing or decreasing near the point $c$, and claim (3) follows.

As a general remark, we note here that $\theta^{-}, \theta^{+}$are uniquely determined in $(0,2 \pi]$ since $B^{-}(\varphi, a)=\left(-\varphi^{\prime}(a), \varphi(a)\right) \neq(0,0)$ and $B^{+}(\varphi, b)=\left(\varphi^{\prime}(b), \varphi(b)\right) \neq(0,0)$.

In the case where $n=0$, we have either $\varphi(x)>0$ in $(a, b)$ or $\varphi(x)<0$ in $(a, b)$. If $\varphi(x)>0$ in $(a, b)$, then we have either $\varphi(a)>0$ or else $\varphi(a)=0$ and $\varphi^{\prime}(a)>0$, which implies that $\theta^{-} \in(0, \pi]$. Similarly, if $\varphi(x)>0$ in $(a, b)$, then we have either $\varphi(b)>0$ or else $\varphi(b)=0$ and $\varphi^{\prime}(b)<0$, which ensures that $\theta^{+} \in(0, \pi]$. In a similar way, we may conclude that if $\varphi(x)<0$ in $(a, b)$, then $\theta^{-}, \theta^{+} \in(\pi, 2 \pi]$.

Now we assume that $n \geq 1$. Let $\left\{x_{i}\right\}_{i=1}^{n}$ be the increasing sequence of the zeroes of $\varphi$ in $(a, b)$. The argument above for $n=0$, applied to the intervals $\left(a, x_{1}\right)$ and $\left(x_{n}, b\right)$, shows that if $\varphi(x)>0$ in $\left(a, x_{1}\right)$, then $\theta^{-} \in(0, \pi]$, if $\varphi(x)<0$ in $\left(a, x_{1}\right)$, then $\theta^{-} \in(\pi, 2 \pi]$, if $\varphi(x)>0$ in $\left(x_{n}, b\right)$, then $\theta^{+} \in(0, \pi]$, and if $\varphi(x)<0$ in $\left(x_{n}, b\right)$, then $\theta^{+} \in(\pi, 2 \pi]$. By induction, we can show that if $n$ is an even integer, then the function $\varphi$ has the same sign on the intervals $\left(a, x_{1}\right)$ and $\left(x_{n}, b\right)$, and if $n$ is odd, then $\varphi$ has opposite signs on $\left(a, x_{1}\right)$ and $\left(x_{n}, b\right)$. Thus, if $n$ is even and $\varphi(x)>0$ in $\left(a, x_{1}\right)$, then $\theta^{-}, \theta^{+} \in(0, \pi]$, if $n$ is even and $\varphi(x)<0$ in $\left(a, x_{1}\right)$, then $\theta^{-}, \theta^{+} \in(\pi, 2 \pi]$, if $n$ is odd and $\varphi(x)>0$ in $\left(a, x_{1}\right)$, then $\theta^{-} \in(0, \pi]$ and $\theta^{+} \in(\pi, 2 \pi]$, and if $n$ is odd and $\varphi(x)<0$ in $\left(a, x_{1}\right)$, then $\theta^{-} \in(\pi, 2 \pi]$ and $\theta^{+} \in(0, \pi]$. Thus claim (4) is valid. 


\subsection{The strong maximum principle}

We now state the strong maximum principle for $F$. For a proof, see [25, Theorem 2.6].

Proposition 13 Assume that (F1) and (F2) hold. Let $u, v \in W^{2,1}(a, b)$ satisfy

$$
F[u] \geq F[v] \text { and } u \leq v \text { in }(a, b) .
$$

Then either $u \equiv v$ in $[a, b]$ or else $u<v$ in $(a, b), \max \left\{(v-u)(a),(v-u)^{\prime}(a)\right\}>0$ and $\max \left\{(v-u)(b),-(v-u)^{\prime}(b)\right\}>0$.

The following lemma is a consequence of the proposition above, which is useful in the arguments below.

Lemma 14 Assume that (F1)-(F2) hold. Let $u, v \in W^{2,1}(a, b)$ and $\theta_{u}^{-}, \theta_{u}^{+} \in$ $(0,2 \pi]$ and $\theta_{v}^{-}, \theta_{v}^{+} \in(0, \pi]$. Assume that $F[v] \leq F[u]$ in $(a, b)$ and

$$
B(u, a, b) \in \overline{L\left(\theta_{u}^{-}, \theta_{u}^{+}\right)} \quad \text { and } \quad B(v, a, b) \in L\left(\theta_{v}^{-}, \theta_{v}^{+}\right)
$$

Furthermore, assume that $v>0$ in $(a, b)$ and $\sup _{(a, b)} u / v=1$ and that $\theta_{u}^{-} \geq \theta_{v}^{-}$ and $\theta_{u}^{+} \geq \theta_{v}^{+}$. Then we have $u=v$ on $[a, b]$.

In the statement above, $\overline{L\left(\theta_{u}^{-}, \theta_{u}^{+}\right)}$denotes the closure of the set $L\left(\theta_{u}^{-}, \theta_{u}^{+}\right)$in $\mathbb{R}^{4}$. We remark that $\overline{L\left(\theta_{u}^{-}, \theta_{u}^{+}\right)}=\overline{l\left(\theta_{u}^{-}\right)} \times \overline{l\left(\theta_{u}^{+}\right)}=\left(\{(0,0)\} \cup l\left(\theta_{u}^{-}\right)\right) \times\left(\{(0,0)\} \cup l\left(\theta_{u}^{+}\right)\right)$.

Proof We argue by contradiction and suppose that $u \not \equiv v$. By assumption, we have $\sup _{(a, b)} u / v=1$ and thus $u \leq v$ on $[a, b]$. By the strong maximum principle, Proposition 13, we see that

$$
\left\{\begin{array}{l}
v(x)>u(x) \quad \text { for all } x \in(a, b), \\
\max \left\{v(a)-u(a), v^{\prime}(a)-u^{\prime}(a)\right\}>0, \\
\max \left\{v(b)-u(b), u^{\prime}(b)-v^{\prime}(b)\right\}>0
\end{array}\right.
$$

From this we observe that if $v(a)=u(a)$, then $-v^{\prime}(a)<-u^{\prime}(a)$ and that if $v(b)=$ $u(b)$, then $v^{\prime}(b)<u^{\prime}(b)$. Hence, if $v(a)=u(a)>0$, then we obtain

$$
\theta_{v}^{-}=\cos ^{-1} \frac{-v^{\prime}(a)}{\sqrt{v^{\prime}(a)^{2}+v(a)^{2}}}>\cos ^{-1} \frac{-u^{\prime}(a)}{\sqrt{u^{\prime}(a)^{2}+u(a)^{2}}}=\theta_{u}^{-}
$$

[see also Fig. 4], which is a contradiction. Similarly, if $v(b)=u(b)>0$, we obtain $\theta_{v}^{+}>\theta_{u}^{+}$, a contradiction.

Note by (29) that $u(x) / v(x)<1$ for all $x \in(a, b)$. If $v(a)>u(a)$ and $v(b)>$ $u(b)$, then we have $v(x)>u(x)$ for all $x \in[a, b]$. This shows that $\sup _{(a, b)} u / v<1$, which is a contradiction.

What remains is the case where either $v(a)=u(a)=0$ or $v(b)=u(b)=0$, which can be divided into three subcases: (1) $v(a)=u(a)=0$ and $v(b)=u(b)=0$, 
Fig. 4 Comparison of angles

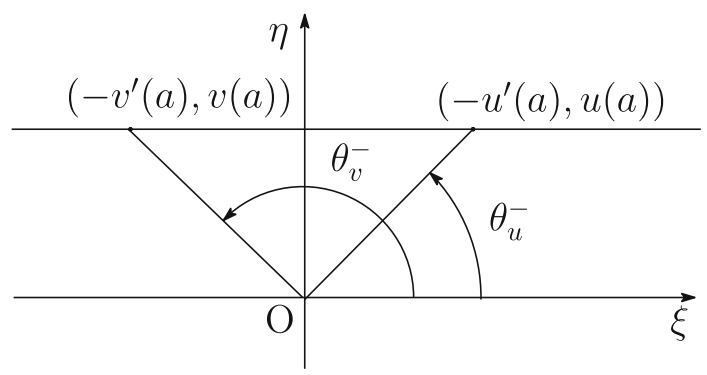

(2) $v(a)=u(a)=0$ and $v(b)>u(b)$ or (3) $v(a)>u(a)$ and $v(b)=u(b)=0$. If $v(a)=u(a)=0$, then we have $v^{\prime}(a)>u^{\prime}(a), \theta_{v}=\pi$ and $v^{\prime}(a)>0$, a consequence of the inclusion $B^{-}(v, a) \in l(\pi)$, and moreover, by l'Hôpital's rule,

$$
\lim _{x \rightarrow a+0} \frac{u(x)}{v(x)}=\frac{u^{\prime}(a)}{v^{\prime}(a)}<1 .
$$

Similarly, if $v(b)=u(b)=0$, then

$$
\lim _{x \rightarrow b-0} \frac{u(x)}{v(x)}<1 .
$$

It is now easy to see in each subcases (1)-(3) that

$$
\lim _{x \rightarrow a+0} \frac{u(x)}{v(x)}<1 \quad \text { and } \quad \lim _{x \rightarrow b-0} \frac{u(x)}{v(x)}<1,
$$

which yields the inequality, $\sup _{(a, b)} u / v<1$, a contradiction, and concludes that $u \equiv v$ on $[a, b]$.

\subsection{The maximum and comparison principles}

In what follows we denote by $F^{+}$the function on $\mathbb{R}^{3} \times[a, b]$ given by $F^{+}(m, p, r, x)=$ $P^{+}(m)+\beta(x)|p|+\gamma(x)|r|$, where $\beta, \gamma \in L^{1}(a, b)$ are from (F2). Remark that $F^{+}$ satisfies (F1)-(F3).

Theorem 15 Assume that $(\mathrm{F} 1)$ and $(\mathrm{F} 2)$ hold and $F[0] \equiv 0$. Let $\mu \in \mathbb{R}$ and $\theta=$ $\left(\theta^{-}, \theta^{+}\right) \in(0, \pi]^{2}$, and assume that there exists a function $\psi \in W^{2,1}(a, b)$ such that

$$
\left\{\begin{array}{l}
F^{+}[\psi]+\mu \psi \leq 0, \quad F^{+}[\psi]+\mu \psi \neq \equiv 0 \text { and } \psi>0 \quad \text { in }(a, b), \\
B(\psi, a, b) \in L(\theta) .
\end{array}\right.
$$

If $u \in W^{2,1}(a, b)$ satisfies

$$
\left\{\begin{array}{l}
F[u]+\mu u \geq 0 \quad \text { in }(a, b), \\
B(u, a, b) \in \overline{L(\tau)} \text { for some } \tau \in\left[\theta^{-}, 2 \pi\right] \times\left[\theta^{+}, 2 \pi\right],
\end{array}\right.
$$

then $u \leq 0$ in $(a, b)$. 
Proof Suppose to the contrary that $\sup _{(a, b)} u>0$. Set $\rho=\sup _{(a, b)} u / \psi$. It is easily checked that, if $\psi(a)>0$ and $\psi(b)>0$, then $0<\rho<\infty$ and $\sup _{(a, b)} u / \rho \psi=$ 1. Setting $\tau=\left(\tau^{-}, \tau^{+}\right)$, we observe that if $\psi(a)=0$, then, since $B(\psi, a, b) \in$ $L\left(\theta^{-}, \theta^{+}\right)$, we have $\pi=\theta^{-} \leq \tau^{-}, \psi^{\prime}(a)>0$ and $u(a) \leq 0$, and, moreover, by using l'Hôpital's rule, we get

$\lim _{x \rightarrow a+0} \frac{u(x)}{\psi(x)}=\frac{u^{\prime}(a)}{\psi^{\prime}(a)}<\infty$ if $u(a)=0 \quad$ and $\quad \lim _{x \rightarrow a+0} \frac{u(x)}{\psi(x)}=-\infty$ otherwise.

Similarly, we have $\lim _{x \rightarrow b-0} u(x) / \psi(x)<\infty$. Thus, we have $0<\rho<\infty$ and $\sup _{(a, b)} u / \rho \psi=1$.

We note that, since $F[0]=0, F^{+}[\rho \psi]=\rho F^{+}[\psi]$ and $F[u]+\mu u \geq 0 \geq$ $F^{+}[\rho \psi]+\mu(\rho \psi) \geq F[\rho \psi]+\mu \rho \psi$ in $(a, b)$. Hence, using Lemma 14, we have $u \equiv \rho \psi$ on $[a, b]$ while $F[u]+\mu u \geq 0 \not \equiv F[\rho \psi]+\mu(\rho \psi)$ in $(a, b)$, which is a contradiction.

The proof above can be used to show that the following maximum principle holds. The proof will be left to the reader.

Theorem 16 Assume that $(\mathrm{F} 1)-(\mathrm{F} 3)$ hold. Let $\theta=\left(\theta^{-}, \theta^{+}\right) \in(0, \pi]^{2}$, and assume that there exists a function $\psi \in W^{2,1}(a, b)$ such that

$$
\left\{\begin{array}{l}
F[\psi] \leq 0, \quad F[\psi] \neq \equiv 0 \text { and } \psi>0 \quad \text { in }(a, b) \\
B(\psi, a, b) \in L(\theta)
\end{array}\right.
$$

If $u \in W^{2,1}(a, b)$ satisfies

$$
\left\{\begin{array}{l}
F[u] \geq 0 \text { in }(a, b), \\
B(u, a, b) \in \overline{L(\tau)} \text { for some } \tau \in\left[\theta^{-}, 2 \pi\right] \times\left[\theta^{+}, 2 \pi\right],
\end{array}\right.
$$

then $u \leq 0$ in $(a, b)$.

A typical situation where (31) is satisfied is the following: let $(\nu, \psi)$ be a positive principal eigenpair of (4)-(5). If we choose $\mu<v$, then $F[\psi]+\mu \psi<0$ in $(a, b)$. That is, condition (31) holds with this choice of $\mu$ and the function $F(m, p, r, x)$ replaced by $F(m, p, r, x)+\mu r$.

We give two propositions concerning the comparison principle for ODE (4).

Theorem 17 Assume that (F1) and (F2) hold. Let $\mu \in \mathbb{R}$ and $\theta=\left(\theta^{-}, \theta^{+}\right) \in$ $(0, \pi]^{2}$, and assume that there exists a function $\psi \in W^{2,1}(a, b)$ such that $(30)$ holds. If $v, w \in W^{2,1}(a, b)$ satisfy

$$
\left\{\begin{array}{l}
F[v]+\mu v \geq F[w]+\mu w \quad \text { in }(a, b), \\
B(v-w, a, b) \in \overline{L(\tau)} \text { for some } \tau \in\left[\theta^{-}, 2 \pi\right] \times\left[\theta^{+}, 2 \pi\right]
\end{array}\right.
$$

then $v \leq w$ in $(a, b)$. 
Proof We set $u=v-w$ and observe that

$$
0 \leq F[v]-F[w]+\mu(v-w) \leq F^{+}[u]+\mu u \quad \text { in }(a, b)
$$

We apply Theorem 15, with the function $F$ replaced by the function $F^{+}$, to conclude that $v \leq w$ in $(a, b)$.

Proposition 18 Assume that $(\mathrm{F} 1)-(\mathrm{F} 3)$ hold. Let $\theta=\left(\theta^{-}, \theta^{+}\right) \in(0, \pi]^{2}$, and assume that there exists a function $\psi \in W^{2,1}(a, b)$ that satisfies (31). If $v, w \in$ $W^{2,1}(a, b)$ satisfy

$\left\{\begin{array}{l}F[w] \leq 0, \quad F[w] \leq F[v] \text { and } w>0 \text { in }(a, b), \quad B(w, a, b) \in L(\theta), \quad \text { and } \\ B(v, a, b) \in \overline{L(\tau)} \text { for some } \tau \in\left[\theta^{-}, 2 \pi\right] \times\left[\theta^{+}, 2 \pi\right]\end{array}\right.$

then $v \leq w$ in $(a, b)$.

Proof Suppose to the contrary that $\max _{[a, b]}(v-w)>0$. Since $B^{-}(w, a) \in l\left(\theta^{-}\right)$ and $B^{-}(v, a) \in \overline{l\left(\tau^{-}\right)}$, where $\tau^{-} \in\left[\theta^{-}, 2 \pi\right]$, if $w(a)=0$, then we have $\theta^{-}=$ $\pi, w^{\prime}(a)>0$ and $v(a) \leq 0$. Similarly, if $w(b)=0$, then we have $w^{\prime}(b)<0$ and $v(b) \leq 0$. Therefore, setting $\rho=\sup _{(a, b)} v / w$, we have $\rho \in(1, \infty)$. We set $f=F[v]$ and $g=F[w]$, and note that $f, g \in L^{1}(a, b), g \leq 0$ and $g \leq f$ in $[a, b]$, and

$$
F[\rho w]=\rho g \leq g \leq f=F[v] \quad \text { in }(a, b),
$$

and apply the strong maximum principle, Lemma 14 , to obtain $\rho w \equiv v$ on $[a, b]$. Hence, the inequalities above are indeed equalities, from which we get $(\rho-1) g \equiv 0$ and $F[w]=0$ in $(a, b)$. By Theorem 16 , we get $w \leq 0$ on $[a, b]$, which is a contradiction. The proof is complete.

The following proposition states that for any $\theta \in(0, \pi)^{2}$, condition (30) holds for some $\mu \in \mathbb{R}$ and $\psi \in W^{2,1}(a, b)$. In the following three propositions we always assume without further comment that (F2) holds.

Proposition 19 For any $\theta=\left(\theta^{-}, \theta^{+}\right) \in(0, \pi)^{2}$ there exist $\mu \in \mathbb{R}$ and $\psi \in$ $W^{2,1}(a, b)$ that satisfy (30).

We need two lemmas for the proof of the proposition above.

For $M>0$ we define $\gamma_{M} \in L^{1}(a, b)$ by $\gamma_{M}(x)=(\gamma(x)-M)_{+}$.

Lemma 20 For any $\varepsilon>0$ there exist $M>0, \alpha \geq 0$ and $v \in W^{1,1}(a, b)$ such that

$$
\lambda v^{\prime}+\beta|v|+\gamma_{M}=\alpha \quad \text { and }|v|<\varepsilon \quad \text { in }(a, b), \quad \text { and } \quad v(a)=v(b)=0 .
$$

Proof We fix any $M>0$. For each $\alpha \geq 0$ we solve the initial value problem

$$
\lambda v^{\prime}+\beta|v|+\gamma_{M}=\alpha \quad \text { in }(a, b) \quad \text { and } \quad v(a)=0 .
$$


We denote the unique solution $v(x)$ of (32) by $v(x ; \alpha)$. We set $\bar{\beta}(x)=$ $\operatorname{sgn}(v(x ; \alpha)) \beta(x)$ for $x \in(a, b)$,

$B(c, d)=\int_{c}^{d} \lambda^{-1} \beta(t) \mathrm{d} t \quad$ and $\quad \bar{B}(c, d)=\int_{c}^{d} \lambda^{-1} \bar{\beta}(t) \mathrm{d} t \quad$ for $a \leq c \leq d \leq b$,

and note that the function $v(x)=v(x ; \alpha)$ satisfies

$$
v^{\prime}(x)+\lambda^{-1} \bar{\beta}(x) v(x)=\lambda^{-1}\left(\alpha-\gamma_{M}(x)\right) \quad \text { in }(a, b) .
$$

Consequently, we have

$$
v(x ; \alpha)=\lambda^{-1} \mathrm{e}^{-\bar{B}(a, x)} \int_{a}^{x} \mathrm{e}^{\bar{B}(a, t)}\left(\alpha-\gamma_{M}(t)\right) \mathrm{d} t \quad \text { for } x \in[a, b] .
$$

Hence, noting that $|\bar{B}(c, d)| \leq B(c, d) \leq B(a, b)$ for $a \leq c<d \leq b$, we have for all $x \in[a, b]$,

$$
\begin{aligned}
v(x ; \alpha) & =\lambda^{-1} \int_{a}^{x} \mathrm{e}^{-\bar{B}(t, x)}\left(\alpha-\gamma_{M}(t)\right) \mathrm{d} t \\
& \leq \lambda^{-1}\left(\int_{a}^{b} \mathrm{e}^{B(a, b)} \alpha \mathrm{d} t-\int_{a}^{x} \mathrm{e}^{-B(a, b)} \gamma_{M}(t) \mathrm{d} t\right) \\
& \leq \lambda^{-1}\left((b-a) \mathrm{e}^{B(a, b)} \alpha-\mathrm{e}^{-B(a, b)}\left\|\gamma_{M}(t)\right\|_{L^{1}(a, x)}\right),
\end{aligned}
$$

and

$$
v(x ; \alpha) \geq \lambda^{-1}\left((x-a) \mathrm{e}^{-B(a, b)} \alpha-\mathrm{e}^{B(a, b)}\left\|\gamma_{M}\right\|_{L^{1}(a, b)}\right) .
$$

In particular, we get $v(b ; 0) \leq 0$, and $v(b ; \alpha) \geq 0$ if $\alpha \geq(b-$ $a)^{-1} \mathrm{e}^{2 B(a, b)}\left\|\gamma_{M}\right\|_{L^{1}(a, b)}$. The general theory of ODE or an application of the Gronwall inequality assures that the function $\alpha \mapsto v(b ; \alpha)$ is continuous on $[0, \infty)$. Thus, the intermediate value theorem assures that there exists $\alpha_{M} \in[0, \infty)$ such that $v\left(b ; \alpha_{M}\right)=0$ and, moreover, $\alpha_{M} \leq(b-a)^{-1} \mathrm{e}^{2 B(a, b)}\left\|\gamma_{M}\right\|_{L^{1}(a, b)}$.

Now, we fix any $\varepsilon>0$. By (33) and (34), we get

$$
\begin{aligned}
\max _{x \in[a, b]}\left|v\left(x ; \alpha_{M}\right)\right| & \leq \lambda^{-1} \mathrm{e}^{B(a, b)} \max \left\{(b-a) \alpha_{M},\left\|\gamma_{M}\right\|_{L^{1}(a, b)}\right\} \\
& \leq \lambda^{-1} \mathrm{e}^{3 B(a, b)}\left\|\gamma_{M}\right\|_{L^{1}(a, b)} .
\end{aligned}
$$

Note that $\lim _{M \rightarrow \infty}\left\|\gamma_{M}\right\|_{L^{1}(a, b)}=0$, and choose $M>0$ so that $\lambda^{-1} \mathrm{e}^{3 B(a, b)}$ $\left\|\gamma_{M}\right\|_{L^{1}(a, b)}<\varepsilon$, which implies that $\left|v\left(x ; \alpha_{M}\right)\right|<\varepsilon$ for all $x \in[a, b]$. The proof is complete.

Lemma 21 There exist $\mu \in \mathbb{R}$ and $w \in W^{2,1}(a, b)$ such that

$$
\left\{\begin{array}{l}
P^{+}[w]+\beta\left|w^{\prime}\right|+\gamma|w|+\mu w<0 \quad \text { in }(a, b) \\
w>0 \text { on }[a, b] \text { and } w^{\prime}(a)=w^{\prime}(b)=0
\end{array}\right.
$$


Proof According to Lemma 20, there exist $v \in W^{1,1}(a, b), M>0$, and $\alpha \geq 0$ such that

$\lambda v^{\prime}+\beta|v|+\gamma_{M}=\alpha \quad$ and $\quad|v| \leq \frac{1}{8(b-a)} \quad$ in $(a, b), \quad$ and $\quad v(a)=v(b)=0$.

We define $w \in W^{2,1}(a, b)$ by

$$
w(x)=\frac{1}{2}+\int_{a}^{x} v(t) \mathrm{d} t .
$$

Observe that $\lambda w^{\prime \prime}+\beta(x)\left|w^{\prime}\right|+\gamma_{M}=\alpha$ in $(a, b), w^{\prime}(a)=w^{\prime}(b)=0,1 / 4<$ $w<1$ in $(a, b)$ and $\left(w^{\prime \prime}\right)_{+} \leq \lambda^{-1} \alpha$ in $(a, b)$. Therefore,

$$
\begin{aligned}
F^{+}[w] \leq & \lambda w^{\prime \prime}+(\Lambda-\lambda)\left(w^{\prime \prime}\right)_{+}+\beta\left|w^{\prime}\right|+\gamma w \leq \lambda w^{\prime \prime}+\left(\lambda^{-1} \Lambda-1\right) \alpha \\
& +\beta\left|w^{\prime}\right|+\gamma_{M} w+M w \\
\leq & \lambda w^{\prime \prime}+\beta\left|w^{\prime}\right|+\gamma_{M}+\left(\lambda^{-1} \Lambda-1\right) \alpha \\
& +M w \leq \lambda^{-1} \Lambda \alpha+M w<\left(4 \lambda^{-1} \Lambda \alpha+M\right) w .
\end{aligned}
$$

The pair of the constant $\mu=-4 \lambda^{-1} \Lambda \alpha-M$ and function $w$ has all the required properties.

Proof of Proposition 19 We set $p^{-}=\cos \theta^{-} / \sin \theta^{-}$and $p^{+}=-\cos \theta^{+} / \sin \theta^{+}$, and select a function $\zeta \in C^{2}([a, b])$ and a constant $\delta \in(0,(b-a) / 2)$ such that

$$
\zeta(x) \begin{cases}=\mathrm{e}^{p^{-}(x-a)} & \text { for } x \in[a, a+\delta), \\ =\mathrm{e}^{p^{+}(x-b)} & \text { for } x \in(b-\delta, b], \\ >0 & \text { for } x \in[a+\delta, b-\delta] .\end{cases}
$$

Set also $\eta(x)=1 / \zeta(x)$ for $x \in[a, b]$. We define the function $F_{\zeta}^{+}$on $\mathbb{R}^{3} \times[a, b]$ by

$$
F_{\zeta}^{+}(m, p, r, x)=\zeta(x) F^{+}\left(\eta(x) m+2 \eta^{\prime}(x) p+\eta^{\prime \prime}(x) r, \eta(x) p+\eta^{\prime}(x) r, \eta(x) r, x\right),
$$

and calculate that for any $(m, p, r, x) \in \mathbb{R}^{3} \times(a, b)$,

$$
\begin{aligned}
F_{\zeta}^{+} & (m, p, r, x) \\
\leq & \zeta(x)\left\{P^{+}(\eta(x) m)+P^{+}\left(2\left|\eta^{\prime}(x) p\right|+\left|\eta^{\prime \prime}(x) r\right|\right)\right. \\
& \left.+\beta(x)\left(|\eta(x) p|+\left|\eta^{\prime}(x) r\right|\right)+\gamma(x)|\eta(x) r|\right\} \\
& \leq P^{+}(m)+\zeta(x)\left\{\Lambda\left(2\left|\eta^{\prime}(x) p\right|+\left|\eta^{\prime \prime}(x) r\right|\right)+\beta(x)\left(\eta(x)|p|+\left|\eta^{\prime}(x) r\right|\right)\right. \\
& +\gamma(x) \eta(x)|r|\} \\
& \leq P^{+}(m)+\beta_{\zeta}(x)|p|+\gamma_{\zeta}(x)|r|,
\end{aligned}
$$


where $\beta_{\zeta}, \gamma_{\zeta} \in L^{1}(a, b)$ are given by

$$
\begin{aligned}
& \beta_{\zeta}(x)=\beta(x)+2 \Lambda \zeta(x)\left|\eta^{\prime}(x)\right| \text { and } \\
& \gamma_{\zeta}(x)=\gamma(x)+\Lambda \zeta(x)\left|\eta^{\prime \prime}(x)\right|+\beta(x) \zeta(x)\left|\eta^{\prime}(x)\right| .
\end{aligned}
$$

By Lemma 21, with $\beta$ and $\gamma$ replaced by $\beta_{\zeta}$ and $\gamma_{\zeta}$, we can choose $\mu \in \mathbb{R}$ and $w \in W^{2,1}(a, b)$ so that (35) holds.

We set $\psi=\eta w$ on $[a, b]$, and note that $\psi>0$ on $[a, b]$,

$$
\begin{aligned}
0 & >F_{\zeta}^{+}[w]+\mu w \\
& =\zeta\left(F^{+}\left((\eta w)^{\prime \prime},(\eta w)^{\prime}, \eta w, x\right)+\mu \psi\right)=\zeta\left(F^{+}[\psi]+\mu \psi\right) \quad \text { in }(a, b),
\end{aligned}
$$

and hence, $F^{+}[\psi]+\mu \psi<0$ in $(a, b)$. Observe that $0=w^{\prime}(a)=p^{-} \psi(a)+\psi^{\prime}(a)$ and therefore

$B^{-}(\psi, a)=\left(-\psi^{\prime}(a), \psi(a)\right)=\left(p^{-} \psi(a), \psi(a)\right)=\frac{\psi(a)}{\sin \theta^{-}}\left(\cos \theta^{-}, \sin \theta^{-}\right) \in l\left(\theta^{-}\right)$.

Similarly, we get $B^{+}(\psi, b) \in l\left(\theta^{+}\right)$. The proof is complete.

\subsection{Basic estimates}

Lemma 22 Assume that (F1) and (F2) hold. Let $(c, d)$ be a subinterval of $(a, b), z \in$ $[c, d]$ and $v \in W^{2,1}(c, d)$. If $F[v] \in L^{1}(a, b)$, then

$$
\begin{aligned}
\|v\|_{W^{1, \infty}(c, d)} \leq & \left(|v(z)|+\left|v^{\prime}(z)\right|+\lambda^{-1}\left(\|F[v]\|_{L^{1}(c, d)}\right.\right. \\
& \left.\left.+\|F[0]\|_{L^{1}(c, d)}\right)\right) \exp \left(\left\|\lambda^{-1}(\beta+\gamma)+1\right\|_{L^{1}(c, d)}\right) .
\end{aligned}
$$

Proof Let $g_{F}$ be the function as in Lemma 12. Setting $f=F[v]$, we have

$$
v^{\prime \prime}(x)=g_{F}\left(v^{\prime}(x), v(x), f(x), x\right) \text { in }(c, d),
$$

and moreover,

$$
\left|v^{\prime \prime}(x)\right| \leq \lambda^{-1}\left(\beta(x)\left|v^{\prime}(x)\right|+\gamma(x)|v(x)|+|f(x)|+|F[0](x)|\right) \quad \text { in }(c, d) .
$$

Hence, setting $h(x)=|v(x)|+\left|v^{\prime}(x)\right|$ for $x \in[c, d]$, we have

$$
\begin{aligned}
h(x) & \leq h(z)+\lambda^{-1}\left|\int_{z}^{x}\left[(\beta(t)+\lambda)\left|v^{\prime}(t)\right|+\gamma(t)|v(t)|+|f(t)|+|F[0](t)|\right] \mathrm{d} t\right| \\
& \leq h(z)+\left|\int_{z}^{x}(\hat{\alpha}(t) h(t)+\hat{f}(t)) \mathrm{d} t\right|,
\end{aligned}
$$


where $\hat{\alpha}(t):=\lambda^{-1}(\beta(t)+\gamma(t))+1$ and $\hat{f}(t):=\lambda^{-1}(|f(t)|+|F[0](t)|)$. By the Gronwall inequality, we get

$$
\begin{aligned}
h(x) & \leq h(z) \exp \left(\left|\int_{z}^{x} \hat{\alpha}(t) \mathrm{d} t\right|\right)+\left|\int_{z}^{x} \exp \left(\left|\int_{t}^{x} \hat{\alpha}(s) \mathrm{d} s\right|\right) \hat{f}(t) \mathrm{d} t\right| \\
& \leq\left(h(z)+\|\hat{f}\|_{L^{1}(c, d)}\right) \exp \left(\|\hat{\alpha}\|_{L^{1}(c, d)}\right) .
\end{aligned}
$$

for all $x \in[c, d]$, which completes the proof.

Lemma 23 Assume that (F1) and (F2) hold. Let $(c, d)$ be a subinterval of $(a, b)$ and set

$$
C_{0}=\exp \left(\left\|\lambda^{-1}(\beta+\gamma)+1\right\|_{L^{1}(c, d)}\right)
$$

Let $v \in W^{2,1}(c, d)$, and assume that $F[v] \in L^{1}(a, b)$, that either $v(c)=0$ or $v(d)=0$ and that the inequality, $\left|v^{\prime}(z)\right| \leq \sigma|v(z)|$, holds for some $\sigma \geq 0$ and $z \in[c, d]$. Then

$$
\begin{aligned}
& \|v\|_{W^{1, \infty}(c, d)} \\
& \quad \leq C_{0}\left((1+\sigma)(d-c)\|v\|_{W^{1, \infty}(c, d)}+\lambda^{-1}\|F[v]\|_{L^{1}(c, d)}+\|F[0]\|_{L^{1}(c, d)}\right) .
\end{aligned}
$$

Furthermore, if $C_{0}(1+\sigma)(d-c)<1$, then

$$
\|v\|_{W^{1, \infty}(c, d)} \leq \frac{C_{0} \lambda^{-1}\left(\|F[v]\|_{L^{1}(c, d)}+\|F[0]\|_{L^{1}(c, d)}\right)}{1-C_{0}(1+\sigma)(d-c)}
$$

Proof By the assumption that $v(c)=0$ or $v(d)=0$, we get $|v(x)| \leq\left\|v^{\prime}\right\|_{L^{1}(c, d)}$ for all $x \in[c, d]$. Since $\left|v^{\prime}(z)\right| \leq \sigma|v(z)|$, we have $|v(z)|+\left|v^{\prime}(z)\right| \leq(1+\sigma)|v(z)|$. Hence, we get

$$
|v(z)|+\left|v^{\prime}(z)\right| \leq(1+\sigma)|v(z)| \leq(1+\sigma)\left\|v^{\prime}\right\|_{L^{1}(c, d)} \leq(1+\sigma)(d-c)\|v\|_{W^{1, \infty}(c, d)} .
$$

We combine this with Lemma 22, to get

$$
\begin{aligned}
& \|v\|_{W^{1, \infty}(c, d)} \\
& \quad \leq C_{0}\left((1+\sigma)(d-c)\|v\|_{W^{1, \infty}(c, d)}+\lambda^{-1}\left(\|F[v]\|_{L^{1}(c, d)}+\|F[0]\|_{L^{1}(c, d)}\right)\right) .
\end{aligned}
$$

Moreover, it follows that if $C_{0}(1+\sigma)(d-c)<1$, then

$$
\|v\|_{W^{1, \infty}(c, d)} \leq \frac{C_{0} \lambda^{-1}\left(\|F[v]\|_{L^{1}(c, d)}+\|F[0]\|_{L^{1}(c, d)}\right)}{1-C_{0}(1+\sigma)(d-c)}
$$


Lemma 24 Assume that (F1) and (F2) hold and $F[0] \equiv 0$. Let $\left\{f_{k}\right\}_{k \in \mathbb{N}} \subset$ $L^{1}(a, b),\left\{\mu_{k}\right\}_{k \in \mathbb{N}} \subset \mathbb{R}$ and $\left\{v_{k}\right\}_{k \in \mathbb{N}} \subset W^{2,1}(a, b)$. Assume that for some $f \in$ $L^{1}(a, b)$ and $\mu \in \mathbb{R}$,

$$
\lim _{k \rightarrow \infty} f_{k}=f \quad \text { strongly in } L^{1}(a, b) \quad \text { and } \quad \lim _{k \rightarrow \infty} \mu_{k}=\mu \text {, }
$$

and that for any $k \in \mathbb{N}, v_{k}$ is a solution of $F\left[v_{k}\right]+\mu_{k} v_{k}+f_{k}=0$ in $(a, b)$. If

$$
\sup _{k \in \mathbb{N}[a, b]} \min \left(\left|v_{k}\right|+\left|v_{k}^{\prime}\right|\right)<\infty
$$

then $\left\{v_{k}\right\}$ has a convergent subsequence in $W^{2,1}(a, b)$ and the limit $v$ of the subsequence is a solution of $F[v]+\mu v+f=0$ in $(a, b)$.

Proof Choose a sequence $\left\{z_{k}\right\}_{k \in \mathbb{N}} \subset[a, b]$ so that $\left|v_{k}\left(z_{k}\right)\right|+\left|v_{k}^{\prime}\left(z_{k}\right)\right|=$ $\min _{[a, b]}\left(\left|v_{k}\right|+\left|v_{k}^{\prime}\right|\right)$ for all $k \in \mathbb{N}$. By Lemma 22 , we have

$$
\left\|v_{k}\right\|_{W^{1, \infty}(a, b)} \leq M_{k}\left(\left|v_{k}\left(z_{k}\right)\right|+\left|v_{k}^{\prime}\left(z_{k}\right)\right|+\lambda^{-1}\left\|f_{k}\right\|_{L^{1}(a, b)}\right)
$$

for all $k \in \mathbb{N}$, where $M_{k}=\exp \left(\left\|\lambda^{-1}\left(\beta+\gamma+\left|\mu_{k}\right|\right)+1\right\|_{L^{1}(a, b)}\right)$. Thus, we see from (37) that

$$
\sup _{k \in \mathbb{N}}\left\|v_{k}\right\|_{W^{1, \infty}(a, b)}<\infty .
$$

According to (36) or Lemma 12, we have

$$
\begin{aligned}
& \left|v_{k}^{\prime \prime}(x)\right| \\
& \quad \leq \lambda^{-1}\left(\beta(x)\left|v_{k}^{\prime}(x)\right|+\left(\gamma(x)+\left|\mu_{k}\right|\right)\left|v_{k}(x)\right|+\left|f_{k}(x)\right|\right) \quad \text { in }(a, b) \quad \text { for all } k \in \mathbb{N},
\end{aligned}
$$

which shows together with (38) that $\left\{v_{k}^{\prime \prime}\right\}$ is uniformly integrable on $(a, b)$ and hence the sequence $\left\{v_{k}\right\}$ is relatively compact in $C^{1}([a, b])$. Observe by using Lemma 12 that for any $k, \ell \in \mathbb{N}$,

$$
\begin{aligned}
\left|v_{k}^{\prime \prime}-v_{\ell}^{\prime \prime}\right| & =\left|g_{F}\left(v_{k}^{\prime}, v_{k},-\mu_{k} v_{k}-f_{k}, x\right)-g_{F}\left(v_{\ell}^{\prime}, v_{\ell},-\mu_{\ell} v_{\ell}-f_{\ell}, x\right)\right| \\
& \leq \lambda^{-1}\left(\beta\left|v_{k}^{\prime}-v_{\ell}^{\prime}\right|+\gamma\left|v_{k}-v_{\ell}\right|+\left|\mu_{k} v_{k}-\mu_{\ell} v_{\ell}\right|+\left|f_{k}-f_{\ell}\right|\right) \text { in }(a, b),
\end{aligned}
$$

where $g_{F}$ is the function given by Lemma 12. Thus, we find that $\left\{v_{k}^{\prime \prime}\right\}$ contains a strongly convergent subsequence in $L^{1}(a, b)$ and the sequence $\left\{v_{k}\right\}$ is relatively compact in the strong topology of $W^{2,1}(a, b)$. Let $v$ be a limit point of the sequence $\left\{v_{k}\right\}$. That is, $v=\lim _{j \rightarrow \infty} v_{k_{j}}$ in the strong topology of $W^{2,1}(a, b)$ for some subsequence $\left\{v_{k_{j}}\right\}$ of $\left\{v_{k}\right\}$. By passing once again to a subsequence if necessary, we may assume that $\left(v^{\prime \prime}(x), v^{\prime}(x), v(x)\right)=\lim _{j \rightarrow \infty}\left(v_{k_{j}}^{\prime \prime}(x), v_{k_{j}}^{\prime}(x), v_{k_{j}}(x)\right)$ for a.e. $x \in(a, b)$. It is now easy to conclude that $v^{\prime \prime}(x)=g_{F}\left(v^{\prime}(x), v(x),-\mu(x) v(x)-f(x)\right)$ a.e. in $(a, b)$, which assures that $v$ is a solution of $F[v]+\mu v+f=0$ in $(a, b)$. 


\section{Proofs of the main results in the one-dimensional case}

In this section, we prove Theorems 2-5. Throughout this section we assume that (F1)-(F3) hold.

\subsection{Comparison of eigenvalues}

We give here the proof of Theorem 4.

Proof of Theorem 4 For $i=1,2$, set $\theta_{i}=\left(\theta_{i}^{-}, \theta_{i}^{+}\right)$and let $\left(\mu_{i}, \varphi_{i}\right) \in \mathbb{R} \times$ $W^{2,1}(a, b)$ be an $n_{i}$ th order eigenpairs of (4)-(5), with $\left(\theta^{-}, \theta^{+}\right)$replaced by $\left(\theta_{i}^{-}, \theta_{i}^{+}\right)$, and assume that $\left(n_{1}, \theta_{1}\right) \leq\left(n_{2}, \theta_{2}\right)$.

We argue by contradiction, and suppose that $\mu_{1}>\mu_{2}$.

Let $\left\{x_{j}\right\}_{j=0}^{n_{1}+1},\left\{y_{j}\right\}_{j=0}^{n_{2}+1}$ be the increasing sequences of points in $[a, b]$ such that $x_{0}=y_{0}=a, x_{n_{1}+1}=y_{n_{2}+1}=b$, and the $x_{j}$, with $0<j<n_{1}+1$, and the $y_{j}$, with $0<j<n_{2}+1$, are zeroes of the functions $\varphi_{1}$ and $\varphi_{2}$, respectively.

By assumption, there exist nonnegative integers $k^{-}$and $k^{+}$such that (11)-(15) hold.

Consider first the case when $k^{-}=k^{+}=0$. We note that $n_{1}=n_{2}$ and $x_{n_{1}+1}=$ $y_{n_{1}+1}=b$, set $m=\min \left\{j \in\left\{1, \ldots, n_{1}+1\right\}: y_{j} \leq x_{j}\right\}$ and observe that $x_{m-1} \leq$ $y_{m-1}<y_{m} \leq x_{m}$. Since $k^{-}=0$, which implies together with (12) that $\varphi_{1} \varphi_{2}>0$ in $\left(a, \min \left\{x_{1}, y_{1}\right\}\right)$, and $\varphi_{1}$ and $\varphi_{2}$ change sign at $x_{j}$ and $y_{j}$ for every $0<j<n_{1}+1$, respectively, we see that $\varphi_{1} \varphi_{2}>0$ in $\left(y_{m-1}, y_{m}\right)$. Furthermore, observe that, if $y_{m-1}=a$, then $B^{-}\left(\varphi_{1}, a\right) \in l\left(\theta_{1}^{-}\right)$and $B^{-}\left(\varphi_{2}, a\right) \in l\left(\theta_{2}^{-}\right)$and, if $y_{m}=b$, then $B^{+}\left(\varphi_{1}, y_{m}\right) \in l\left(\theta_{1}^{+}\right)$and $B^{+}\left(\varphi_{2}, y_{m}\right) \in l\left(\theta_{2}^{+}\right)$, and that $i\left(\theta_{1}^{-}\right)=i\left(\theta_{2}^{-}\right), \theta_{1}^{-} \leq$ $\theta_{2}^{-}, i\left(\theta_{1}^{+}\right)=i\left(\theta_{2}^{+}\right)$and $\theta_{1}^{+} \leq \theta_{2}^{+}$.

Consider next the case when either $k^{-}=0$ and $k^{+}>0$ or $k^{+}=0$ and $k^{-}>0$. In view of (S2), we may assume that $k^{-}=0$ and $k^{+}>0$. We have $n_{2}>n_{1}$ and $y_{n_{1}+1}<b=x_{n_{1}+1}$. As above, we set $m=\min \left\{j \in\left\{1, \ldots, n_{1}+1\right\}: y_{j} \leq x_{j}\right\}$ and observe that $\varphi_{1} \varphi_{2}>0$ in $\left(y_{m-1}, y_{m}\right), y_{m}<b$ and, if $y_{m-1}=a$, then $i\left(\theta_{1}^{-}\right)=i\left(\theta_{2}^{-}\right)$ and $\theta_{1}^{-} \leq \theta_{2}^{-}$.

Consider the case when $k^{-}>0$ and $k^{+}>0$. If $k^{-}$is an odd integer, that is, $\varphi_{1} \varphi_{2}<0$ in $\left(a, \min \left\{x_{1}, y_{1}\right\}\right)$, then we note that $y_{n_{1}+2}<y_{n_{1}+3} \leq y_{n_{1}+k^{-}+k^{+}+1}=x_{n_{1}+1}=b$, set $m=\min \left\{j \in\left\{2, \ldots, n_{1}+2\right\}: y_{j} \leq x_{j-1}\right\}$ and observe that $\varphi_{1} \varphi_{2}>0$ in $\left(y_{m-1}, y_{m}\right)$ and $a<y_{m-1}<y_{m}<b$. Similarly, if $k^{-}$is an even integer, that is, $\varphi_{1} \varphi_{2}>0$ in $\left(a, \min \left\{x_{1}, y_{1}\right\}\right)$, then we note that $y_{n_{1}+3}<y_{n_{1}+4} \leq y_{n_{1}+k^{-}+k^{+}+1}=$ $b=x_{n_{1}+1}$, set $m=\min \left\{j \in\left\{3, \ldots, n_{1}+3\right\}: y_{j} \leq x_{j-2}\right\}$ and observe that $\varphi_{1} \varphi_{2}>0$ in $\left(y_{m-1}, y_{m}\right)$ and $a<y_{m-1}<y_{m}<b$.

Thus, in all possible cases there exists a nonempty subinterval $(c, d) \subset[a, b]$ having the properties: (1) $\varphi_{1} \varphi_{2}>0$ in $(c, d)$, (2) if $c=a$, then $i\left(\theta_{1}^{-}\right)=i\left(\theta_{2}^{-}\right)$and $\theta_{1}^{-} \leq \theta_{2}^{-}$, (3) if $c \neq a$, then $\varphi_{2}(c)=0$ (4) if $d \neq b$, then $\varphi_{2}(d)=0$ and (4) if $d=b$, then $i\left(\theta_{1}^{+}\right)=i\left(\theta_{2}^{+}\right)$and $\theta_{1}^{+} \leq \theta_{2}^{+}$.

By the symmetry (S1) we may assume that $\varphi_{1}>0$ and $\varphi_{2}>0$ in $(c, d)$. As a consequence, we have $F\left[\varphi_{1}\right]+\mu_{2} \varphi_{1}<0$ in $(c, d)$. 
We choose $\tau_{i}^{-}, \tau_{i}^{+} \in(0, \pi]$, with $i=1,2$, so that $B\left(\varphi_{i}, c, d\right) \in L\left(\tau_{i}^{-}, \tau_{i}^{+}\right)$for $i=1,2$. Note that if $c=a$, then $\tau_{i}^{-}=\theta_{i}^{-}$for $i=1,2$, if $c>a$, then $\varphi_{2}(c)=0$ and $\tau_{1}^{-} \leq \tau_{2}^{-}=\pi$, if $d=b$, then $\tau_{i}^{+}=\theta_{i}^{+}$for $i=1,2$, and if $d<b$, then $\varphi_{2}(d)=0$ and $\tau_{1}^{+} \leq \tau_{2}^{+}=\pi$. We use the maximum principle, Theorem 16 with the interval $(a, b)$ and the function $F(m, p, r, x)$ replaced by $(c, d)$ and $F(m, p, r, x)+\mu_{2} r$, respectively, to obtain $\varphi_{2} \leq 0$ on $[c, d]$, which is a contradiction. Thus, we see that the inequality, $\mu_{1} \leq \mu_{2}$, holds.

Applying Theorem 4 twice, under the assumptions (F1)-(F3), for any admissible $(n, \theta) \in \mathbb{N}_{0} \times(0,2 \pi]^{2}$, an $n$th order eigenvalue of (4)-(5) is unique.

\subsection{Existence of principal eigenpairs}

We prove the existence of principal eigenpairs of (4)-(5) in this subsection.

Lemma 25 There exist $c \in(a, b]$ and $(\mu, v) \in \mathbb{R} \times W^{2,1}(a, c)$ such that

$$
F[v]+\mu v \geq 0 \text { and } v>0 \text { in }(a, c), \quad \text { and } \quad v(a)=v(c)=0 .
$$

According to [25], there exist a positive principal eigenpair of (4) with the Dirichlet boundary condition, and the lemma above follows from this observation. But, for the reader's convenience, we give a proof of the lemma above.

Proof Let $c \in(a, b]$ be a constant to be fixed later. Set $f=\beta+\gamma$,

$$
\begin{aligned}
M & =\frac{1}{c-a} \int_{a}^{c} \mathrm{~d} r \int_{a}^{r} f(t) \mathrm{d} t \quad \text { and } \\
w(x) & =\int_{a}^{x}\left(\int_{a}^{r} f(t) \mathrm{d} t-M\right) \mathrm{d} r \text { for } x \in[a, c] .
\end{aligned}
$$

Clearly, we have $w(a)=w(c)=0,0 \leq M \leq\|f\|_{L^{1}(a, c)}$,

$$
w^{\prime}(x)=\int_{a}^{x} f(t) \mathrm{d} t-M \text { for all } x \in[a, c],
$$

$w^{\prime \prime}=f$ in $(a, c), w \in W^{2,1}(a, c)$ and $\left\|w^{\prime}\right\|_{L^{\infty}(a, c)} \leq\|f\|_{L^{1}(a, c)}$.

Observe moreover that for all $x \in[a, c]$,

$0 \leq-w(x) \leq\left\|w^{\prime}\right\|_{L^{\infty}(a, c)} \min \{|x-a|,|x-c|\} \leq\|f\|_{L^{1}(a, c)} \min \{|x-a|,|x-c|\}$.

Recalling that the inequality, $\sin x \geq(2 / \pi) \min \{|x|,|x-\pi|\}$, holds for all $x \in[0, \pi]$, we see that

$$
-w(x) \leq \frac{(c-a)\|f\|_{L^{1}(a, c)}}{2} \sin \frac{\pi(x-a)}{c-a} \text { for all } x \in[a, c] .
$$


Hence, setting $d=(c-a)\|f\|_{L^{1}(a, c)}$ and $\chi(x)=d \sin (\pi(x-a) /(c-a))$ for $x \in[a, c]$, we have $\chi(x)+w(x) \geq \chi(x) / 2>0$ for all $x \in(a, c)$.

We set $v=w+\chi$ on $[a, c]$ and observe that $v(a)=v(c)=0, v(x) \geq \chi(x) / 2>$ 0 for all $x \in(a, c)$. Using the inequalities $w^{\prime \prime} \geq 0, \chi^{\prime \prime} \leq 0$ and $v \leq \chi$ in $(a, b)$ and (F2), we get for a.e. $x \in(a, c)$,

$$
\begin{aligned}
F[v](x) & \geq \lambda w^{\prime \prime}(x)+\Lambda \chi^{\prime \prime}(x)-\beta(x)\left|v^{\prime}(x)\right|-\gamma(x) v(x) \\
& \geq f(x)\left(\lambda-\left(\left|v^{\prime}(x)\right|+\chi(x)\right)\right)+\Lambda \chi^{\prime \prime}(x) \\
& \geq\left\{\lambda-\left(\|f\|_{L^{1}(a, c)}+\frac{\pi d}{c-a}+d\right)\right\} f(x)-\Lambda\left(\frac{\pi}{c-a}\right)^{2} \chi(x) \\
& \geq\left\{\lambda-(1+\pi+c-a)\|f\|_{L^{1}(a, c)}\right\} f(x)-2 \Lambda\left(\frac{\pi}{c-a}\right)^{2} v(x)
\end{aligned}
$$

Now select $c \in(a, b]$ so that $(1+\pi+c-a)\|f\|_{L^{1}(a, c)} \leq \lambda$, put $\mu=2 \Lambda \pi^{2} /(c-$ $a)^{2}$, and conclude that $v$ satisfies (39).

Proof of Theorem 2 (2) in the case $n=0$ In view of the symmetry (S1), we need only to prove the existence of a principal eigenpair $(\mu, \varphi)$ in the case where $\theta=\left(\theta^{-}, \theta^{+}\right) \in$ $(0, \pi]^{2}$.

We treat first the case where $\theta \in(0, \pi)^{2}$.

Given a constant $\mu \in \mathbb{R}$, we consider the initial value problem

$$
F[u]+\mu u=0 \quad \text { in }(a, b) \quad \text { and } \quad\left(u(a), u^{\prime}(a)\right)=\left(\sin \theta^{-},-\cos \theta^{-}\right) .
$$

Thanks to Lemma 12, we know that this problem has a unique solution $u_{\mu} \in$ $W^{2, q}(a, b)$. Proposition 1 assures that $\left(u_{\mu}(x), u_{\mu}^{\prime}(x)\right) \neq 0$ for all $x \in[a, b]$. We may choose $\theta_{\mu} \in(0,2 \pi]$ so that $B^{+}\left(u_{\mu}, b\right) \in l\left(\theta_{\mu}\right)$. We should note that $B^{-}\left(u_{\mu}, a\right) \in l\left(\theta^{-}\right)$.

Next, let $(c, \mu, v) \in(a, b) \times \mathbb{R} \times W^{2,1}(a, b)$ be those from Lemma 25 . We denote this constant $\mu$ by $\nu_{1}$. We show that

$$
\min _{[a, b]} u_{\mu} \leq 0 \quad \text { for all } \mu>v_{1}
$$

We prove this by contradiction. Fix any $\mu>v_{1}$ and suppose that $\min _{[a, b]} u_{\mu}>0$. Accordingly, we have $F\left[u_{\mu}\right]+v_{1} u_{\mu}<0 \leq F[v]+v_{1} v$ and $v>0$ in $(a, c), B^{-}(v, a) \in l(\pi), B^{-}\left(u_{\mu}, a\right) \in l\left(\theta^{-}\right)$, and $v(c)=0$. Also, since $u_{\mu}>0$ in $[a, b]$, we have $B^{+}\left(u_{\mu}, c\right) \in l\left(\theta_{c}\right)$ for some $\theta_{c} \in(0, \pi)$. We apply the maximum principle, Theorem 16 with the interval $(a, b)$ and the function $F(m, p, r, x)$ replaced by $(a, c)$ and $F(m, p, r, x)+v_{1} r$, respectively, to obtain $\max _{[a, c]} v \leq 0$, which is a contradiction. Hence, (41) is valid.

Now, let $(\mu, \psi) \in \mathbb{R} \times W^{2,1}(a, b)$ be the pair given by Proposition 19 . We refer this constant $\mu$ as $v_{0}$ in what follows. We show that

$$
\min _{[a, b]} u_{\mu}>0 \text { and } \theta_{\mu}<\theta^{+} \text {for all } \mu \leq v_{0} .
$$


Let $\mu \leq v_{0}$, and we first show that $\min _{[a, b]} u_{\mu}>0$. Suppose to the contrary that $\min _{[a, b]} u_{\mu} \leq 0$ and choose $d \in(a, b]$ such that $u_{\mu}>0$ in $(a, d)$ and $u_{\mu}(d)=0$. Observe that $\psi>0, F[\psi]+\mu \psi \leq F^{+}[\psi]+v_{0} \psi \leq 0=F\left[u_{\mu}\right]+\mu u_{\mu}$ and $F[\psi]+\mu \psi \not \equiv 0$ in $(a, d), B^{-}(\psi, a), B^{-}\left(u_{\mu}, a\right) \in l\left(\theta^{-}\right)$, and $u_{\mu}(d)=0$. We also remark by Proposition 13, applied to $u \equiv 0$ and $v=\psi$, that $B^{+}(\psi, d) \in l\left(\theta_{d}\right)$ for some $\theta_{d} \in(0, \pi]$ since $\left(\psi(x), \psi^{\prime}(x)\right) \neq(0,0)$ holds for any $x \in[a, b]$. Indeed, we just need to put $\theta_{d}=\Theta\left(-\psi^{\prime}(d), \psi(d)\right)$ and see (3) for the definition of $\Theta$. Hence, by the maximum principle, we get $\max _{[a, d]} u_{\mu} \leq 0$, which is a contradiction. This proves that $\min _{[a, b]} u_{\mu}>0$ for all $\mu \leq v_{0}$. This and (41), in particular, show that $v_{0}<v_{1}$.

We next show that if $\mu \leq v_{0}$, then $\theta_{\mu}<\theta^{+}$. Indeed, if we fix any $\mu \leq v_{0}$ and suppose to the contrary that $\theta_{\mu} \geq \theta^{+}$, then, by Theorem 16 , we obtain $\max _{[a, b]} u_{\mu} \leq 0$, a contradiction.

In view of a classical result on the continuous dependence of the solutions $u_{\mu}$ of (40) in $\mu$, we know that the function $\mu \mapsto \min _{[a, b]} u_{\mu}$ is continuous on $\mathbb{R}$. By (41) and (42), there exists a $\bar{\mu} \in\left(v_{0}, v_{1}\right]$ such that

$$
\min _{[a, b]} u_{\bar{\mu}}=0 \quad \text { and } \quad \min _{[a, b]} u_{\mu}>0 \text { for all } \mu<\bar{\mu} \text {. }
$$

Since $\left(u_{\mu}(x), u_{\mu}^{\prime}(x)\right) \neq 0$ for all $x \in[a, b]$, we deduce that $u_{\bar{\mu}}(b)=0$ and $u_{\bar{\mu}}>0$ in $[a, b)$. Consequently, we have $\theta_{\bar{\mu}}=\pi$. The continuous curve $\mu \mapsto\left(u_{\mu}^{\prime}(b), u_{\mu}(b)\right)$, with $\mu \in\left[v_{0}, \bar{\mu}\right)$, starts at a point on the line $l\left(\theta_{v_{0}}\right)$, where $\theta_{v_{0}}<\theta^{+}$, lies in the upper half-plane $\left\{(x, y) \in \mathbb{R}^{2}: y>0\right\}$, and, as $\mu \rightarrow \bar{\mu}$, approaches to the line $l(\pi)$. This simple geometric observation assures that there exists $\mu \in\left(v_{0}, \bar{\mu}\right)$ such that $B^{+}\left(u_{\mu}, b\right) \in l\left(\theta^{+}\right)$.

We now treat the general case where $\theta \in(0, \pi]^{2}$. We choose two nondecreasing sequences $\left\{\theta_{n}^{-}\right\}_{n \in \mathbb{N}},\left\{\theta_{n}^{+}\right\}_{n \in \mathbb{N}} \subset(0, \pi)$ such that

$$
\lim _{n \rightarrow \infty} \theta_{n}^{-}=\theta^{-} \quad \text { and } \quad \lim _{n \rightarrow \infty} \theta_{n}^{+}=\theta^{+} .
$$

According to the argument above, for each $n \in \mathbb{N}$, we may choose a principal eigenpair $\left(\mu_{n}, u_{n}\right) \in \mathbb{R} \times W^{2, q}(a, b)$ of (4)-(5), with $\left(\theta^{-}, \theta^{+}\right)$replaced by the pair $\left(\theta_{n}^{-}, \theta_{n}^{+}\right)$. By multiplying by positive constants, we may assume that $\left\|u_{n}\right\|_{W^{1, \infty}(a, b)}=1$ for all $n \in \mathbb{N}$. By inequality (41), we deduce that $\mu_{n} \leq v_{1}$, and by Theorem 4 , we have $\mu_{n} \geq \mu_{1}$ for all $n \in \mathbb{N}$. Hence, we have $\mu_{1} \leq \mu_{n} \leq \nu_{1}$ for all $n \in \mathbb{N}$ and we see that $\left\{\mu_{n}\right\}_{n \in \mathbb{N}}$ has a convergent subsequence $\left\{\mu_{n_{k}}\right\}_{k \in \mathbb{N}}$. By Lemma 24, we may assume that $\left\{u_{n_{k}}\right\}$ converges to a function $u \in W^{2,1}(a, b)$ and the function $u$ satisfies (4). Since $\left\{u_{n_{k}}\right\}$ is convergent in $C^{1}([a, b])$, we see that

$$
\lim _{k \rightarrow \infty} B\left(u_{n_{k}}, a, b\right)=B(u, a, b) .
$$

This assures that $B(u, a, b) \in \overline{L(\theta)}$. It is clear that $u \geq 0$ in $(a, b)$ and $\|u\|_{W^{1, \infty}(a, b)}=1$. By Proposition 1 , we have $\left(u(x), u^{\prime}(x)\right) \neq(0,0)$ in $[a, b]$. Hence, we see that $B(u, a, b) \in L(\theta)$ and $u>0$ on $(a, b)$. Thus, $(\mu, u)$ is a principal eigenpair of (4)-(5). Finally, we note by Lemma 12 that $u \in W^{2, q}(a, b)$. 


\subsection{Higher order eigenpairs in the one-dimensional case}

We give first the proof of Theorem 2 (1).

Proof of Theorem 2 (1) Let $\left(\mu_{i}, \varphi_{i}\right)$, with $i=1,2$, be both $n$th order eigenpairs of (4)-(5).

As remarked after the proof of Theorem 4, we know that $\mu_{1}=\mu_{2}$. In what follows we assume that

$$
\left\|\varphi_{1}\right\|_{L^{\infty}(a, b)}=\left\|\varphi_{2}\right\|_{L^{\infty}(a, b)},
$$

and prove that $\varphi_{1}=\varphi_{2}$.

We treat first the case where $n=0$. We have either $\theta^{-}, \theta^{+} \in(0, \pi]$ or $\theta^{-}, \theta^{+} \in$ $(\pi, 2 \pi]$. Thanks to (S1), we need only to consider the case where $\theta^{-}, \theta^{+} \in(0, \pi]$. We have $\varphi_{1}>0$ and $\varphi_{2}>0$ in $(a, b)$. We set $\rho:=\sup _{(a, b)} \varphi_{1} / \varphi_{2}$ and observe, as in the first part of the proof of Theorem 15, that $\rho \in(0, \infty)$. Obviously, we have $\rho \varphi_{2}(x) \geq \varphi_{1}(x)$ for all $x \in[a, b]$. By the strong maximum principle, Lemma 14, we get $\rho \varphi_{2}=\varphi_{1}$ on $[a, b]$. Hence, we see by (43) that $\rho=1$ and conclude that $\varphi_{1}=\varphi_{2}$ on $[a, b]$.

Next, we assume that $n \geq 1$. Let $\left\{x_{j}\right\}_{j=0}^{n+1}$ and $\left\{y_{j}\right\}_{j=0}^{n+1}$ be the increasing sequences of points in $[a, b]$ such that $x_{0}=y_{0}=a, x_{n+1}=y_{n+1}=b$, and the $x_{j}$ and $y_{j}$, with $1 \leq j \leq n$ are zeroes of $\varphi_{1}$ and $\varphi_{2}$ in $(a, b)$, respectively. We may assume, by interchanging the role of $\varphi_{1}$ and $\varphi_{2}$ if needed, that $x_{1} \leq y_{1}$. Using the argument in the previous step, with the interval $(a, b)$ replaced by $\left(x_{0}, x_{1}\right)$, we deduce that $\rho_{1} \varphi_{2}=\varphi_{1}$ on $\left[x_{0}, x_{1}\right]$ for some $\rho_{1} \in(0, \infty)$, which implies that $x_{1}=y_{1}$. Repeating the same argument as above, with $(a, b)$ replaced by $\left(x_{1}, x_{2}\right), \ldots,\left(x_{n}, x_{n+1}\right)$ in this order, we obtain $x_{j}=y_{j}$ and $\varphi_{1}=\rho_{j} \varphi_{2}$ in $\left[x_{j-1}, x_{j}\right]$ for some $\rho_{j} \in(0, \infty)$ and all $2 \leq j \leq n+1$. Since $\varphi_{i} \in C^{1}([a, b])$ and $\varphi_{i}^{\prime}\left(x_{j}\right) \neq 0$ for all $1 \leq j \leq n$ and $i=1,2$, we see that $\rho_{1}=\rho_{2}=\cdots=\rho_{n+1}$. Using (43), we get $\rho_{j}=1$ for all $1 \leq j \leq n+1$ and, therefore, $\varphi_{1} \equiv \varphi_{2}$ in $[a,, b]$. This completes the proof.

The following lemma states that the $n$th order eigenvalue $\mu^{n}(\theta, c, d)$ and its eigenfunction depend continuously on the angles $\theta=\left(\theta^{-}, \theta^{+}\right)$and the interval $(c, d)$.

Lemma 26 Let $n \in \mathbb{N}_{0}, \quad\left\{\left(\theta_{j}^{-}, \theta_{j}^{+}\right)\right\}_{j \in \mathbb{N}} \subset(0,2 \pi]^{2}, \quad\left(\theta^{-}, \theta^{+}\right) \in(0,2 \pi]^{2}$, $\left\{\left(c_{j}, d_{j}\right)\right\}_{j \in \mathbb{N}} \subset[a, b]^{2}, c, d \in[a, b]$ and $\left\{\left(\mu_{j}, \varphi_{j}\right)\right\}_{j \in \mathbb{N}} \subset \mathbb{R} \times W^{2,1}(a, b)$. Assume that $c_{j}<d_{j}$ for all $j \in \mathbb{N}, c<d$ and, for any $j \in \mathbb{N},\left(\mu_{j}, \varphi_{j}\right)$ is an nth eigenpair of (4)-(5), with the interval $(a, b)$ and the pair $\left(\theta^{-}, \theta^{+}\right)$replaced by $\left(c_{j}, d_{j}\right)$ and $\left(\theta_{j}^{-}, \theta_{j}^{+}\right)$, respectively. Furthermore, assume that $\left\|\varphi_{j}\right\|_{W^{1, \infty}\left(c_{j}, d_{j}\right)}=1$, $i\left(\theta_{j}^{-}\right)=i\left(\theta^{-}\right), i\left(\theta_{j}^{+}\right)=i\left(\theta^{+}\right)$for all $j \in \mathbb{N}$, and

$$
\lim _{j \rightarrow \infty}\left(\theta_{j}^{-}, \theta_{j}^{+}, c_{j}, d_{j}\right)=\left(\theta^{-}, \theta^{+}, c, d\right) \text { in } \mathbb{R}^{4} .
$$

Then there exists an $n$th order eigenpair $(\mu, \varphi) \in \mathbb{R} \times W^{2,1}(a, b)$ of (4)-(5), with $(a, b)$ replaced by $(c, d)$, such that

$$
\lim _{j \rightarrow \infty} \mu_{j}=\mu \quad \text { and } \quad \lim _{j \rightarrow \infty} \varphi_{j}=\varphi \quad \text { in } W^{2,1}(a, b) .
$$


A remark here is that in the lemma above, the admissibility of $\left(n, \theta_{j}^{-}, \theta_{j}^{+}\right)$and $\left(n, \theta^{-}, \theta^{+}\right)$is implicitly assumed.

Lemma 27 Let $\left\{\varphi_{k}\right\}_{k \in \mathbb{N}} \subset C^{1}([a, b])$ and $\varphi \in C^{1}([a, b])$. Letn $\in \mathbb{N}_{0},\left\{\left(c_{k}, d_{k}\right)\right\}_{k \in \mathbb{N}}$ $\subset[a, b]^{2},(c, d) \in[a, b]^{2},\left\{\left(\theta_{k}^{-}, \theta_{k}^{+}\right)\right\}_{k \in \mathbb{N}} \subset(0,2 \pi]^{2}$, and $\left(\theta^{-}, \theta^{+}\right) \in(0,2 \pi]^{2}$. Assume that $c_{k}<d_{k}$ for all $k \in \mathbb{N}, c<d$, and $i\left(\theta_{k}^{-}\right)=i\left(\theta^{-}\right)$and $i\left(\theta_{k}^{+}\right)=i\left(\theta^{+}\right)$ for all $k \in \mathbb{N}$, that as $k \rightarrow \infty$,

$$
\varphi_{k} \rightarrow \varphi \quad \text { in } C^{1}([a, b]) \quad \text { and } \quad\left(c_{k}, d_{k}, \theta_{k}^{-}, \theta_{k}^{+}\right) \rightarrow\left(c, d, \theta^{-}, \theta^{+}\right) \quad \text { in } \mathbb{R}^{4},
$$

and that

$$
\left(\varphi(x), \varphi^{\prime}(x)\right) \neq(0,0) \text { for all } x \in[a, b] .
$$

Assume furthermore that every $\varphi_{k}$ has exactly $n$ zeroes in $\left(c_{k}, d_{k}\right)$ and $B\left(\varphi_{k}, c_{k}, d_{k}\right) \in$ $L\left(\theta_{k}^{-}, \theta_{k}^{+}\right)$. Then $\varphi$ has exactly $n$ zeroes in $(a, b)$ and $B(\varphi, c, d) \in L\left(\theta^{-}, \theta^{+}\right)$.

Proof For each $k \in \mathbb{N}$ let $\left\{x_{k, i}\right\}_{i=0}^{n+1} \subset[a, b]$ be the increasing sequence such that $x_{k, 0}=c_{k}, x_{k, n+1}=d_{k}$ and the $x_{k, i}$, with $1 \leq i \leq n$, are zeroes of $\varphi_{k}$ in $\left(c_{k}, d_{k}\right)$.

By passing to a subsequence if necessary, we may assume that, as $k \rightarrow \infty$, the sequence $\left\{\left(x_{k, 0}, \ldots, x_{k, n+1}\right)\right\}_{k \in \mathbb{N}}$ converges to a point $\left(x_{0}, \ldots, x_{n+1}\right)$ in $\mathbb{R}^{n+2}$. Obviously, we have $\varphi\left(x_{i}\right)=0$ for all $i=1, \ldots, n, c=x_{0} \leq x_{1} \leq x_{2} \leq \cdots \leq$ $x_{n} \leq x_{n+1}=d$ and $B(\varphi, c, d) \in \overline{L\left(\theta^{-}, \theta^{+}\right)}$. This inclusion and (46) together yield $B(\varphi, c, d) \in L\left(\theta^{-}, \theta^{+}\right)$. Moreover, because of (46), we may assume that $\left(\varphi_{k}(x), \varphi_{k}^{\prime}(x)\right) \neq(0,0)$ for all $x \in[a, b]$ and $k \in \mathbb{N}$.

We show that

$$
\min \left\{x_{i}-x_{i-1}: i \in\{1, \ldots, n+1\}\right\}>0 .
$$

To the contrary, suppose that $x_{i}=x_{i-1}$ for some $i \in\{1, \ldots, n+1\}$. In the case where $i \in\{2, \ldots, n\}$, by the mean value theorem, for each $k \in \mathbb{N}$ there exists $y_{k} \in\left(x_{k, i-1}, x_{k, i}\right)$ such that $\varphi_{k}^{\prime}\left(y_{k}\right)=0$. Taking the limit as $k \rightarrow \infty$, we get $\left(\varphi\left(x_{i}\right), \varphi^{\prime}\left(x_{i}\right)\right)=0$, which is a contradiction.

Consider next the case where $i=1$. We have now $c=x_{0}=x_{1}$. Hence, we have $\varphi(c)=0$. We may assume by replacing $\varphi_{k}$ and $\varphi$ by $-\varphi_{k}$ and $-\varphi$ if necessary that $i\left(\theta_{k}^{-}\right)=i\left(\theta^{-}\right)=0$ for all $k \in \mathbb{N}$. Since $\varphi(c)=0$, the condition, $\theta^{-} \in(0, \pi]$, implies that $\varphi^{\prime}(c)>0$. Since $\theta_{k}^{-} \in(0, \pi]$ and $\varphi_{k}\left(x_{k, 1}\right)=0$, we have $\varphi_{k}^{\prime}\left(x_{k, 1}\right)<0$ and, in the limit, $\varphi^{\prime}(c) \leq 0$. This contradicts the previous observation that $\varphi^{\prime}(c)>0$. In the case where $i=n+1$, we argue in a way parallel to the case of $i=1$, to obtain a contradiction. Hence, (47) is valid.

Since $\left\{x_{i}\right\}_{i=1}^{n} \subset(c, d)$ consists of distinct zeroes of $\varphi$, the function $\varphi$ has at least $n$ zeroes in $(c, d)$. It remains to show that $\varphi(x) \neq 0$ for all $x \in(c, d) \backslash\left\{x_{1}, \ldots, x_{n}\right\}$. To prove this, we suppose that $\varphi(y)=0$ for some $y \in(c, d) \backslash\left\{x_{1}, \ldots, x_{n}\right\}$. We choose $i \in\{1, \ldots, n+1\}$ and $\delta>0$, in view of (46), so that $x_{i-1}<y-\delta<y<y+\delta<x_{i}$ and $\varphi^{\prime}(x) \neq 0$ for all $x \in(y-\delta, y+\delta)$. Clearly, we have $\varphi(y+\delta) \varphi(y-\delta)<0$ and, by (45), $\varphi_{k}(y+\delta) \varphi_{k}(y-\delta)<0$ for sufficiently large $k \in \mathbb{N}$. The condition, $\varphi_{k}(y+\delta) \varphi_{k}(y-\delta)<0$, ensures that $\varphi_{k}\left(y_{k}\right)=0$ for some $y_{k} \in(y-\delta, y+\delta)$. If $k$ is large enough, then we have $\varphi_{k}\left(y_{k}\right)=0$ and $y_{k} \in\left(x_{k, i-1}, x_{k, i}\right)$. This is a contradiction. That is, $\varphi(x) \neq 0$ for all $x \in(c, d) \backslash\left\{x_{1}, \ldots, n\right\}$. The proof is complete. 
Proof of Lemma 26 In view of (S1), we need only to consider the case when $\theta^{-} \in$ $(0, \pi]$.

We show first that $\left\{\mu_{j}\right\}_{j \in \mathbb{N}}$ is bounded from below. We choose $\tilde{\theta}=\left(\tilde{\theta}^{-}, \tilde{\theta}^{+}\right) \in$ $(0, \pi)^{2}$ so that $\theta^{-}>\tilde{\theta}^{-}$and $\theta^{+}>\tilde{\theta}^{+}$. (Notice that if $n$ is odd, then $\theta^{+}>\pi>t$ for every $t \in(0, \pi)$.) Next choose a principal eigenpair $\left(v_{0}, \varphi_{0}\right) \in \mathbb{R} \times W^{2,1}(a, b)$ of (4)-(5), with the interval $(a, b)$ and the pair $\left(\theta^{-}, \theta^{+}\right)$replaced by $(c, d)$ and $\left(\tilde{\theta}^{-}, \tilde{\theta}^{+}\right)$, respectively. (Recall our convention, explained just after (28), that $\varphi_{0}$ is defined on the interval $[a, b]$ as a solution of (4), with $\mu=\nu_{0}$.) Since $\tilde{\theta} \in(0, \pi)^{2}$, we have $\varphi_{0}>0$ on $[c, d]$. Hence, there exists an open interval $I$, relatively to $[a, b]$, so that $[c, d] \subset I$ and $\varphi_{0}>0$ in $I$. Since $\left(\varphi_{0}^{\prime}(x), \varphi_{0}(x)\right) \neq(0,0)$ in $I$, we may find $t^{-}(x), t^{+}(x) \in(0, \pi)$ for every $x \in I$ such that $B^{-}\left(\varphi_{0}, x\right) \in l\left(t^{-}(x)\right)$ and $B^{+}\left(\varphi_{0}, x\right) \in l\left(t^{+}(x)\right)$, that is, $t^{ \pm}(x)=\Theta\left( \pm \varphi_{0}^{\prime}(x), \varphi_{0}(x)\right)$. Note that $t^{-}(c)=$ $\tilde{\theta}^{-}, t^{+}(d)=\tilde{\theta}^{+}$and $t^{ \pm}(x)$ depend continuously on $x \in I$.

Now by (44) and the choice of $\tilde{\theta}$, for sufficiently large $j \in \mathbb{N}$, we have

$$
c_{j}, d_{j} \in I, \quad t^{-}\left(c_{j}\right)<\theta_{j}^{-} \quad \text { and } t^{+}\left(d_{j}\right)<\theta_{j} .
$$

Noting that $\left(v_{0}, \varphi_{0}\right)$ is a principal eigenpair of (4)-(5) with the interval $\left(c_{j}, d_{j}\right)$ and the angles $\left(t^{-}\left(c_{j}\right), t^{+}\left(d_{j}\right)\right)$ in place of $(a, b)$ and $\left(\theta^{-}, \theta^{+}\right)$, respectively, which implies that $v_{0}=\mu^{0}\left(t^{-}\left(c_{j}\right), t^{+}\left(d_{j}\right), c_{j}, d_{j}\right)$, and that, if $j$ is large enough, then the order relation, $\left(0, t^{-}\left(c_{j}\right), t^{+}\left(d_{j}\right)\right) \leq\left(n, \theta_{j}\right)$, holds, where $\theta_{j}=\left(\theta_{j}^{-}, \theta_{j}^{+}\right)$, we conclude by Theorem 4 that $v_{0}=\mu^{0}\left(t^{-}\left(c_{j}\right), t^{+}\left(d_{j}\right), c_{j}, d_{j}\right) \leq \mu_{j}$ for $j$ sufficiently large and that $\inf _{j \in \mathbb{N}} \mu_{j}>-\infty$.

Next we show that $\left\{\mu_{j}\right\}_{j \in \mathbb{N}}$ is bounded from above. To see this, we fix an increasing sequence $\left\{e_{k}\right\}_{k=0}^{n+1} \subset(c, d)$ and set

$$
\nu_{1}=\max \left\{\mu^{0}\left(\pi, \pi, e_{k-1}, e_{k}\right), \mu^{0}\left(2 \pi, 2 \pi, e_{k-1}, e_{k}\right): k \in\{1, \ldots, n+1\}\right\} .
$$

We select $K \in \mathbb{N}$ so that $c_{j}<e_{0}$ and $d_{j}>e_{n+1}$ for all integers $j \geq K$. Fix any integer $j \geq K$, and let $\left\{x_{j, k}\right\}_{k=0}^{n+1}$ be the increasing sequence such that $x_{j, 0}=$ $c_{j}, x_{j, n+1}=d_{j}$ and the $x_{j, k}$, with $k \in\{1, \ldots, n\}$, are zeroes of $\varphi_{j}$. We define $m=\min \left\{k \in\{1, \ldots, n+1\}: e_{k} \leq x_{j, k}\right\}$ and observe that $m \in\{1, \ldots, n+1\}$ and $\left(e_{m-1}, e_{m}\right) \subset\left(x_{j, m-1}, x_{j, m}\right)$. We use Theorem 4 , with $\left(e_{m-1}, e_{m}\right)$ in place of $(a, b)$, to see that $\mu_{j} \leq \max \left\{\mu^{0}\left(\pi, \pi, e_{m-1}, e_{m}\right), \mu^{0}\left(2 \pi, 2 \pi, e_{m-1}, e_{m}\right)\right\}$. Thus, we obtain $\mu_{j} \leq v_{1}$ for all $j \geq K$, and conclude that $\sup _{j \in \mathbb{N}} \mu_{j}<\infty$.

We may thus choose a convergent subsequence $\left\{\mu_{j_{k}}\right\}_{k \in \mathbb{N}}$ of $\left\{\mu_{j}\right\}$ and set $\mu=$ $\lim _{k \rightarrow \infty} \mu_{j_{k}}$. Furthermore, combining Lemma 22 and the fact that $\left\|\varphi_{j}\right\|_{W^{1, \infty}\left(c_{j}, d_{j}\right)}=$ 1 , we see that $1 \leq \sup _{j \geq 1}\left\|\varphi_{j}\right\|_{W^{1, \infty}(a, b)}<\infty$. By Lemma 24, we may assume by taking a further subsequence if needed that $\left\{\varphi_{j_{k}}\right\}_{k \in \mathbb{N}}$ converges to a function $\varphi$ strongly in $W^{2,1}(a, b)$ and $\varphi$ satisfies $F[\varphi]+\mu \varphi=0$ in $(a, b)$. Set $\theta=\left(\theta^{-}, \theta^{+}\right)$. Since $\left\|\varphi_{j}\right\|_{W^{1, \infty}(a, b)} \geq 1$, we have $\|\varphi\|_{W^{1, \infty}(a, b)} \geq 1$. Hence, $\varphi \not \equiv 0$ in $[a, b]$, which means [see Proposition 1] that $\left(\varphi(x), \varphi^{\prime}(x)\right) \neq(0,0)$ for all $x \in[a, b]$. This combined with the limit relation, $B(\varphi, c, d) \in \overline{L(\theta)}$, assures that $B(\varphi, c, d) \in L(\theta)$. By Lemma 27 , we see that $\varphi$ has exactly $n$ zeroes in $(c, d)$. Therefore, $(\mu, \varphi)$ is an $n$th order eigenpair of (4) and (5), with the interval $(c, d)$ in place of $(a, b)$. In particular, we have $\mu=\mu^{n}(\theta, c, d)$. 
Because of the normalization, $\left\|\varphi_{j}\right\|_{W^{1, \infty}\left(c_{j}, d_{j}\right)}=1$, we obtain $\|\varphi\|_{W^{1, \infty}(c, d)}=1$. By Theorem 2 (1), the $n$th order eigenpair $(\mu, \varphi)$ of (4)-(5), with the interval $(c, d)$ in place of $(a, b)$, is unique. It is a standard observation that this uniqueness assertion combined with the argument above applied to any subsequence of the original sequence $\left\{\left(\mu_{j}, \varphi_{j}\right)\right\}_{j \in \mathbb{N}}$ assures that

$$
\varphi=\lim _{j \rightarrow \infty} \varphi_{j} \quad \text { in } W^{2,1}(a, b) \quad \text { and } \quad \mu=\lim _{j \rightarrow \infty} \mu_{j}
$$

This completes the proof.

Lemma 28 Let $n \in \mathbb{N}_{0}$ and $\theta \in(0,2 \pi]$. If $(n, \theta, \pi)($ resp. $(n, \theta, 2 \pi))$ is admissible, then

$$
\liminf _{\varepsilon \rightarrow 0} \inf _{a \leq c \leq b-\varepsilon} \mu^{n}(\theta, \pi, c, c+\varepsilon)=\liminf _{\varepsilon \rightarrow 0} \inf _{a+\varepsilon \leq d \leq b} \mu^{n}(\pi, \theta, d-\varepsilon, d)=\infty
$$

(resp.

$$
\left.\liminf _{\varepsilon \rightarrow 0} \inf _{a \leq c \leq b-\varepsilon} \mu^{n}(\theta, 2 \pi, c, c+\varepsilon)=\liminf _{\varepsilon \rightarrow 0} \inf _{a+\varepsilon \leq d \leq b} \mu^{n}(2 \pi, \theta, d-\varepsilon, d)=\infty\right) .
$$

Proof In view of (S1) and (S2), we need only to prove that for $\theta \in(0, \pi]$

$$
\liminf _{\varepsilon \rightarrow 0} \inf _{a \leq c \leq b-\varepsilon} \mu^{n}(\theta, \pi, c, c+\varepsilon)=\infty .
$$

We note that if $(n, \theta, \pi)$ is admissible, then $n$ is even, that if $(n, \theta, 2 \pi)$ is admissible, then $n$ is odd, and that the order relations, $(0, \theta, \pi) \leq(2 k, \theta, \pi)$ and $(0, \theta, \pi) \leq$ $(2 k-1, \theta, 2 \pi)$, hold for all $k \in \mathbb{N}$. Hence, we deduce by Theorem 4 that for any $n \in \mathbb{N}_{0}, 0<\varepsilon \leq b-a$ and $c \in[a, b]$ with $c+\varepsilon \leq b$,

$$
\mu^{0}(\theta, \pi, c, c+\varepsilon) \leq \begin{cases}\mu^{n}(\theta, \pi, c, c+\varepsilon) & \text { if }(n, \theta, \pi) \text { is admissible } \\ \mu^{n}(\theta, 2 \pi, c, c+\varepsilon) & \text { if }(n, \theta, 2 \pi) \text { is admissible }\end{cases}
$$

Thus, it is enough to prove that

$$
\liminf _{\varepsilon \rightarrow 0} \inf _{a \leq c \leq b-\varepsilon} \mu^{0}(\theta, \pi, c, c+\varepsilon)=\infty .
$$

To prove (48), we argue by contradiction and suppose that there exist $\left\{\varepsilon_{j}\right\}, c_{j} \in$ $\left[a, b-\varepsilon_{j}\right]$ and $M>0$ such that

$$
\varepsilon_{j} \rightarrow 0, \quad \mu_{j}:=\mu^{0}\left(\theta, \pi, c_{j}, c_{j}+\varepsilon_{j}\right) \leq M \text { for all } j .
$$

Let $\varphi_{j} \in W^{2,1}\left(c_{j}, c_{j}+\varepsilon_{j}\right)$ be an eigenfunction corresponding to $\mu_{j}$ and satisfy $\left\|\varphi_{j}\right\|_{W^{1, \infty}\left(c_{j}, c_{j}+\varepsilon_{j}\right)}=1$. Then we have $\varphi_{j}\left(c_{j}+\varepsilon_{j}\right)=0$ and $F_{j}\left[\varphi_{j}\right]:=F\left[\varphi_{j}\right]+$ $\mu_{j} \varphi_{j}=0$ in $\left(c_{j}, c_{j}+\varepsilon_{j}\right)$. Moreover, $F_{j}$ satisfies $(\mathrm{F} 1)-(\mathrm{F} 3)$ with $\beta_{j}(x):=\beta(x)$ and $\gamma_{j}(x):=\left(\gamma(x)+\mu_{j}\right)_{+}$in place of $\beta$ and $\gamma$. 
If $\theta=\pi$, we may find some $\tau_{j} \in\left(c_{j}, c_{j}+\varepsilon_{j}\right)$ so that $\varphi_{j}^{\prime}\left(\tau_{j}\right)=0$. If $\theta=\pi / 2$, we also have $\varphi_{j}^{\prime}\left(c_{j}\right)=0$. Therefore, when $\theta=\pi$ or $\theta=\pi / 2$, one has $\left|\varphi_{j}^{\prime}\left(z_{j}\right)\right|=$ $0=0 \cdot\left|\varphi_{j}\left(z_{j}\right)\right|$ for some $z_{j} \in\left[c_{j}, c_{j}+\varepsilon_{j}\right]$. On the other hand, if $\theta \neq \pi / 2, \pi$, then the condition, $B^{-}\left(\varphi_{j}, c_{j}\right) \in l(\theta)$, says that the point $(\xi, \eta)=\left(-\varphi_{j}^{\prime}\left(c_{j}\right), \varphi_{j}\left(c_{j}\right)\right)$ lies on the line, $\eta=s \xi$, with the slope $s \in \mathbb{R} \backslash\{0\}$, which yields $\left|\varphi_{j}^{\prime}\left(c_{j}\right)\right| \leq\left|\varphi_{j}\left(c_{j}\right)\right| /|s|$. Thus in general, we have $\left|\varphi_{j}^{\prime}\left(z_{j}\right)\right| \leq \sigma\left|\varphi_{j}\left(z_{j}\right)\right|$ for some $\sigma \geq 0$ and $z_{j} \in\left[c_{j}, c_{j}+\varepsilon_{j}\right]$ where $\sigma$ is independent of $j$.

Now, setting

$$
C_{j}:=\exp \left(\left\|\lambda^{-1}\left(\beta_{j}+\gamma_{j}\right)+1\right\|_{L^{1}\left(c_{j}, c_{j}+d_{j}\right)}\right)
$$

we see that $\left\{C_{j}\right\}$ is bounded due to the definition of $\gamma_{j}$ and $\mu_{j} \leq M$. Thus for sufficiently large $j$, we obtain $C_{j}(1+\sigma) \varepsilon_{j}<1$ and Lemma 23 gives a contradiction:

$$
1=\left\|\varphi_{j}\right\|_{W^{1, \infty}\left(c_{j}, c_{j}+\varepsilon_{j}\right)} \leq \frac{C_{j} \lambda^{-1}}{1-C_{j}(1+\sigma) \varepsilon_{j}} \cdot 0=0 .
$$

Hence, (48) holds.

We now give a proof of Theorem 2 (2) in the case $n \geq 1$, which in turn completes the proof of Theorem 2 .

Proof of Theorem 2 (2) in the general case We have already shown that claim (2) of Theorem 2 holds in the case where $n=0$.

We prove the claim by induction on $n$, and we assume that the claim holds up to $n=k$, with $k \in \mathbb{N}_{0}$. Here we understand that this induction assumption is valid not only on the interval $[a, b]$, but also on any subintervals $[a, c]$ and $[c, b]$, with $c \in(a, b)$. Hence, for any admissible $\left(k+1, \theta^{-}, \theta^{+}\right)$, where $k \in \mathbb{N}_{0}$, and any $c \in(a, b)$, we have a $k$ th order eigenpair $\left(\mu_{c}, \varphi_{c}\right)$ of $(4)$ in $(a, c)$ and a zeroth order eigenpair $\left(v_{c}, \psi_{c}\right)$ of $(4)$ in $(c, b)$ such that $B^{-}\left(\varphi_{c}, a\right) \in l\left(\theta^{-}\right), \varphi_{c}(c)=\psi_{c}(c)=0$ and $B^{+}\left(\psi_{c}, b\right) \in l\left(\theta^{+}\right)$. More precisely, $\left(\mu_{c}, \varphi_{c}\right)\left[\right.$ resp. $\left.\left(v_{c}, \psi_{c}\right)\right]$ is a $k$ th (resp. zeroth) order eigenpair of (4) in $(a, c)$ [resp. in $(c, b)]$ and $B\left(\varphi_{c}, a, c\right) \in L\left(\theta^{-}, \tau^{+}\right)$[resp. $\left.B\left(\psi_{c}, c, b\right] \in L\left(\tau^{-}, \theta^{+}\right)\right)$, where

$$
\left(\tau^{+}, \tau^{-}\right)= \begin{cases}(\pi, 2 \pi) & \text { if } k+i\left(\theta^{-}\right) \text {is even } \\ (2 \pi, \pi) & \text { otherwise }\end{cases}
$$

so that $\left(k, \theta^{-}, \tau^{+}\right)$and $\left(0, \tau^{-}, \theta^{+}\right)$are admissible.

By Lemma 26, the functions $c \mapsto \mu_{c}$ and $c \mapsto v_{c}$ are continuous on $(a, b]$ and $[a, b)$, respectively, and moreover, by Lemma 28 , we have $\lim _{c \rightarrow a+0}\left(\mu_{c}-v_{c}\right)=\infty$ and $\lim _{c \rightarrow b-0}\left(\mu_{c}-v_{c}\right)=-\infty$. It follows by the intermediate value theorem that there exists $c_{1} \in(a, b)$ such that $\mu_{c_{1}}=v_{c_{1}}$. Since $\left(k+1, \theta^{-}, \theta^{+}\right)$is admissible, we have $\varphi_{c_{1}}^{\prime}\left(c_{1}\right) \psi_{c_{1}}^{\prime}\left(c_{1}\right)>0$. We choose a constant $\rho>0$ so that $\varphi_{c_{1}}^{\prime}\left(c_{1}\right)=\rho \psi_{c_{1}}^{\prime}\left(c_{1}\right)$. It is obvious that if we define the function $\chi$ on $[a, b]$ by 


$$
\chi(x)= \begin{cases}\varphi_{c_{1}}(x) & \text { for } x \in\left[a, c_{1}\right], \\ \rho \psi_{c_{1}}(x) & \text { for } x \in\left[c_{1}, b\right]\end{cases}
$$

then $\chi \in W^{2, q}(a, b)$ and $\left(\mu_{c_{1}}, \chi\right)$ is a $(k+1)$ st order eigenpair of (4)-(5). This completes the proof.

Proof of Corollary 3 If $n$ is an even integer, then triplets $\left(n, \theta_{1}^{-}, \theta_{1}^{+}\right)$and $\left(n, \theta_{2}^{-}, \theta_{2}^{+}\right)$ are admissible. Otherwise, $\left(n, \theta_{1}^{-}, \theta_{2}^{+}\right)$and $\left(n, \theta_{2}^{-}, \theta_{1}^{+}\right)$are admissible.

Assume temporarily that $n$ is even. By Theorem 2, there are $n$th order eigenpairs $\left(\mu^{ \pm}, \varphi^{ \pm}\right) \in \mathbb{R} \times W^{2, q}(a, b)$ of $(4)-(5)$, with $\left(\theta^{-}, \theta^{+}\right)$replaced by $\left(\theta_{1}^{-}, \theta_{1}^{+}\right)$and $\left(\theta_{2}^{-}, \theta_{2}^{+}\right)$, respectively. Let $\left\{x_{i}^{ \pm}\right\}_{i=0}^{n+1} \subset[a, b]$ be the increasing sequences such that $x_{0}^{ \pm}=a, x_{n+1}^{ \pm}=b$ and $\varphi^{ \pm}\left(x_{i}^{ \pm}\right)=0$ for all $i \in\{1, \ldots, n\}$. We may assume by multiplying $\varphi^{ \pm}$by positive constants if necessary that $\left\|\varphi^{ \pm}\right\|_{L^{\infty}(a, b)}=1$. With this choice of $\varphi^{ \pm}$and $\left\{x_{i}^{ \pm}\right\}_{i=0}^{n+1}$, the condition (1) is satisfied. Let $(\mu, \varphi) \in \mathbb{R} \times W^{2, q}(a, b)$ be an $n$th order eigenpair of (4) and (10) normalized so that $\|\varphi\|_{L^{\infty}(a, b)}=1$. For some $\delta>0$, we have either $\varphi>0$ in $(a, a+\delta)$ or $\varphi<0$ in $(a, a+\delta)$, and, if $\varphi>0$ in $(a, a+\delta)$ [resp. $\varphi<0$ in $(a, a+\delta)$ ], then $(\mu, \varphi)$ is an $n$th order eigenpair of (4)-(5), with $\left(\theta^{-}, \theta^{+}\right)$replaced by $\left(\theta_{1}^{-}, \theta_{1}^{+}\right)$[resp. $\left.\left(\theta_{2}^{-}, \theta_{2}^{+}\right)\right]$. Theorem 2 ensures that if $(\mu, \varphi)$ is an $n$th order eigenpair of (4)-(5), with $\left(\theta^{-}, \theta^{+}\right)$replaced by $\left(\theta_{1}^{-}, \theta_{1}^{+}\right)$ [resp. $\left.\left(\theta_{2}^{-}, \theta_{2}^{+}\right)\right]$, then we have $(\mu, \varphi)=\left(\mu^{+}, \varphi^{+}\right)$[resp. $\left.(\mu, \varphi)=\left(\mu^{-}, \varphi^{-}\right)\right]$. This shows that the condition (2) is satisfied.

The case where $n$ is odd can be treated similarly to the above, and we skip the details.

\subsection{Characterizations of eigenvalues}

We present here a proof of Theorem 5.

Regarding the symmetry (S1), we remark that for $(\mu, u) \in \mathbb{R} \times W^{2,1}(a, b)$, the equality

$$
(F[u]+\mu u) u=(-F[-(-u)]+\mu(-u))(-u) \text { in }(a, b),
$$

holds and that the inequality (16) [resp. (17)] holds for $(\mu, u) \in \mathbb{R} \times W^{2,1}(a, b)$, then $(\mu,-u)$ satisfies (16) [resp. (17)], with the function $F(m, p, r, x)$ replaced by $-F(-m,-p,-r, x)$. Thus, the symmetry (S1) is valid for both (16) and (17). The symmetry (S2), as well, is obviously valid for both (16) and (17).

Proof of Theorem 5 Let $\varphi \in W^{2,1}(a, b)$ be an eigenfunction corresponding to $\mu^{n}(\theta, a, b)$ and set $\mu=\mu^{n}(\theta, a, b)$.

We prove first the inequality, $\mu \geq \sup E^{-}(n, \theta)$. To do this, we suppose to the contrary that $\mu<\sup E^{-}(n, \theta)$. We may choose an $n$th order solution $(\nu, \psi) \in$ $\mathbb{R} \times W^{2,1}(a, b)$ of (16) and (5) such that $\mu<v$.

Let $\left\{x_{j}\right\}_{j=1}^{n}$ and $\left\{y_{j}\right\}_{j=1}^{n}$ be the increasing sequences of zeroes in $(a, b)$ of $\varphi$ and $\psi$, respectively. We set $x_{0}=y_{0}=a$ and $x_{n+1}=y_{n+1}=b$. As in the proof of Theorem 4 , let $k$ be the smallest $j \in\{1, \ldots, n+1\}$ such that $x_{j} \leq y_{j}$ and note 
that $\left(x_{k-1}, x_{k}\right) \subset\left(y_{k-1}, y_{k}\right)$ and $\varphi \psi>0$ in $\left(x_{k-1}, x_{k}\right)$. In view of the symmetry stated prior to this proof, we need only to treat the case where $\varphi>0$ and $\psi>0$ in $\left(x_{k-1}, x_{k}\right)$.

From (16), since $\mu<v$, we get

$$
F[\psi]+\mu \psi<F[\psi]+\nu \psi \leq 0=F[\varphi]+\mu \varphi \quad \text { in }\left(x_{k-1}, x_{k}\right)
$$

Now, by Theorem 16, we see that $\varphi \leq 0$ in $\left(x_{k-1}, x_{k}\right)$, which is a contradiction. Here we have used the fact that $B\left(\psi, x_{k-1}, x_{k}\right) \in L\left(\theta_{\psi}\right)$ for some $\theta_{\psi}=\left(\theta_{\psi}^{-}, \theta_{\psi}^{+}\right) \in$ $(0, \pi]^{2}$, which is a easy consequence of Proposition 13 , the inequality $\psi>0$ in $\left(x_{k-1}, x_{k}\right)$ and the above inequality. Thus we obtain $\mu \geq \sup E^{-}(n, \theta)$.

Next, we note that $(\mu, \varphi)$ satisfies (16) with equality, which shows that $\mu \in$ $E^{-}(n, \theta)$ and $\mu \leq \max E^{-}(n, \theta)$. Hence, we concludes that $\mu=\max E^{-}(n, \theta)$.

Let $\alpha=(m, \tau) \in \mathbb{N}_{0} \times(0,2 \pi]^{2}$ be admissible and satisfy $\alpha \leq(n, \theta)$. By Theorem 4 , we have $\mu^{m}(\tau, a, b) \leq \mu$ and, as observed above, $\mu^{m}(\tau, a, b)=\max E^{-}(m, \tau)$. Thus, we obtain

$$
\max \widetilde{E}^{-}(n, \theta)=\max _{\alpha \leq(n, \theta)} \max E^{-}(\alpha)=\max E^{-}(n, \theta)=\mu
$$

We prove next the inequality $\mu \leq \inf E^{+}\left(n, \theta^{-}, \theta^{+}\right)$. We suppose to the contrary that $\mu>\inf E^{+}(n, \theta)$. We may choose an $n$th order solution $(v, \psi) \in \mathbb{R} \times W^{2,1}(a, b)$ of (17) and (5) such that $\mu>v$.

Let $\left\{x_{j}\right\}_{j=1}^{n}$ and $\left\{y_{j}\right\}_{j=1}^{n}$ be the increasing sequences of zeroes in $(a, b)$ of $\varphi$ and $\psi$, respectively. We set $x_{0}=y_{0}=a$ and $x_{n+1}=y_{n+1}=b$. Let $k$ be the smallest $j \in\{1, \ldots, n+1\}$ such that $y_{j} \leq x_{j}$ and note that $\left(y_{k-1}, y_{k}\right) \subset\left(x_{k-1}, x_{k}\right)$. We have $\varphi \psi>0$ in $\left(y_{k-1}, y_{k}\right)$. In view of the symmetry (S1), we need only to treat the case where $\varphi>0$ and $\psi>0$ in $\left(y_{k-1}, y_{k}\right)$. From (17), we have

$$
F[\varphi]+\nu \varphi<F[\varphi]+\mu \varphi=0 \leq F[\psi]+\mu \psi \quad \text { in }\left(y_{k-1}, y_{k}\right)
$$

Hence, by Theorem 16 , we get $\psi \leq 0$ in $\left(y_{k-1}, y_{k}\right)$, which is a contradiction. This proves that $\mu \leq \inf E^{+}(n, \theta)$.

Note that $(\mu, \varphi)$ satisfies (17), which ensures that $\mu \in E^{+}(n, \theta)$, and proves that $\mu=\min E^{+}(n, \theta)$. Using Theorem 4 , we easily deduce that $\min \widetilde{E}^{+}(n, \theta)=$ $\min E^{+}(n, \theta)$ and conclude that $\mu=\min \widetilde{E}^{+}(n, \theta)=\min E^{+}(n, \theta)$.

\subsection{Inhomogeneous equations}

We treat now (18) and prove Theorem 6.

We start by noting that for $(\mu, u) \in \mathbb{R} \times W^{2,1}(a, b)$,

$$
\begin{aligned}
-(F[u]+\mu u+\operatorname{sgn}(u) f) & =-F[-(-u)]+\mu(-u)-\operatorname{sgn}(-(-u)) f \\
& =-F[-(-u)]+\mu(-u)+\operatorname{sgn}(-u) f \text { in }(a, b),
\end{aligned}
$$


and therefore that if $(\mu, u)$ satisfies $F[u]+\mu u+\operatorname{sgn}(u) f=0$ in $(a, b)$, then $(\mu, v):=$ $(\mu,-u)$ satisfies $-F[-v]+\mu v+\operatorname{sgn}(v) f=0$ in $(a, b)$. Hence, (18) has the symmetry (S1). Also, it is obvious that (18) has the symmetry (S2).

Proof of Theorem 6 (3) We argue by contradiction and suppose that there were an $n$th order solution $(\mu, u) \in \mathbb{R} \times W^{2,1}(a, b)$ of (18). Note that $u$ satisfies

$$
(F[u]+\mu u) u=-\operatorname{sgn}(u) u f \leq 0 \text { in }(a, b) .
$$

It follows from Theorem 5 that $\mu^{n}(\theta, a, b) \geq \mu$, which implies, together with our assumption on $\mu$, that $\mu=\mu^{n}(\theta, a, b)$.

Let $\varphi$ be an $n$th order eigenfunction corresponding to $\mu^{n}(\theta, a, b)$. Let $\left\{x_{i}\right\}_{i=0}^{n+1}$ and $\left\{y_{i}\right\}_{i=0}^{n+1}$ be the increasing sequences of points in $[a, b]$ such that $x_{0}=y_{0}=a$, $x_{n+1}=y_{n+1}=b$, and the $x_{i}$ and $y_{i}$, with $1 \leq i \leq n$, are zeroes of $u$ and $\varphi$ in $(a, b)$, respectively.

We show that $u$ and $\varphi$ have the same zeroes, that is, $x_{i}=y_{i}$ for all $i \in\{1, \ldots, n\}$. To see this, we assume for the moment that $n \geq 1$ and, to the contrary, suppose that $x_{i} \neq y_{i}$ for some $i \in\{1, \ldots, n\}$. Set $j=\min \left\{i \in\{1, \ldots, n\}: x_{i} \neq y_{i}\right\}$ and $k=j$ if $x_{j}>y_{j}$. If $y_{j}>x_{j}$, then we set $k=\min \left\{i \in\{j+1, \ldots, n+1\}: y_{i} \leq x_{i}\right\}$. (Notice that $j<n+1$ and $\left.x_{n+1}=y_{n+1}\right)$. Observe that $\left(y_{k-1}, y_{k}\right) \subset\left(x_{k-1}, x_{k}\right)$ and the inclusion is strict, that is, $\left(x_{k-1}, x_{k}\right) \neq\left(y_{k-1}, y_{k}\right)$.

In view of (S1), we may assume that $u>0$ and $\varphi>0$ in $\left(y_{k-1}, y_{k}\right)$. We set $\rho=\sup _{\left(y_{k-1}, y_{k}\right)} \varphi / u$. Since $F[u]+\mu u \leq 0$ in $\left(y_{k-1}, y_{k}\right)$, using Proposition 13, we get $\left(u^{\prime}(x), u(x)\right) \neq(0,0)$ for all $x \in\left[y_{k-1}, y_{k}\right]$, from which we deduce that $0<\rho<\infty$. Noting that $F[\rho u]+\mu \rho u \leq 0=F[\varphi]+\mu \varphi$ in $\left(x_{k-1}, x_{k}\right)$ and applying the strong maximum principle, Lemma 14 , we see that $\varphi \equiv \rho u$ in $\left[y_{k-1}, y_{k}\right]$, which implies that $u\left(y_{k-1}\right)=u\left(y_{k}\right)=0$. This is a contradiction since $u>0$ in $\left(x_{k-1}, x_{k}\right)$ and either $y_{k-1}$ or $y_{k}$ belongs to $\left(x_{k-1}, x_{k}\right)$. Thus we conclude that $x_{i}=y_{i}$ for all $i \in\{1, \ldots, n\}$.

We set $c=a$ and $d=b$ if $n=0$ and, otherwise, we choose $k \in\{1, \ldots, n+1\}$ so that $f \not \equiv 0$ in $\left(x_{k-1}, x_{k}\right)$, and set $c=x_{k-1}$ and $d=x_{k}$. We may assume that $\varphi>0$ and $u>0$ in $\left(x_{k-1}, x_{k}\right)$. We note that $F[u]+\mu u \leq 0$ and $F[u]+\mu u \neq 0$ in $(c, d)$ and by Proposition 13 that $B(\varphi, c, d), B(u, c, d) \in L(\tilde{\theta})$ where $\tilde{\theta}=\left(\tilde{\theta}^{-}, \tilde{\theta}^{+}\right), \tilde{\theta}^{-}=\theta^{-}$ if $c=a, \tilde{\theta}^{-}=\pi$ otherwise and $\tilde{\theta}^{+}=\theta^{+}$if $d=b, \tilde{\theta}^{+}=\pi$ otherwise. Thus Theorem 16 gives $\varphi \leq 0$ in $(c, d)$, a contradiction, which shows that there is no $n$th order solution of (18).

Let $a \leq c<d \leq b$ and $(\mu, u) \in W^{2,1}(c, d)$ be a solution of (18), with $a$ and $b$ replaced by $c$ and $d$, respectively. We may extend the domain of definition of $u$ so that $u$ belongs to $W^{2,1}(a, b)$ and satisfies $F[u]+\mu u+f=0$ in $(a, c) \cup(d, b)$. Based on the observation above, as in the case of (4), we agree henceforth that the original $u \in W^{2,1}(c, d)$ is identified with the extended $u \in W^{2,1}(a, b)$.

Lemma 29 Let $\left\{f_{k}\right\}_{k \in \mathbb{N}} \subset L^{1}(a, b), f \in L^{1}(a, b),\left\{\left(c_{k}, d_{k}\right)\right\}_{k \in \mathbb{N}} \subset[a, b]^{2},(c, d)$ $\in[a, b]^{2},\left\{\theta_{k}\right\}_{k \in \mathbb{N}} \subset(0,2 \pi]^{2}, \theta \in(0,2 \pi]^{2},\left\{\mu_{k}\right\}_{k \in \mathbb{N}} \subset \mathbb{R}$, and $\mu \in \mathbb{R}$. Assume that $f_{k} \geq 0$ in $(a, b)$ and $c_{k}<d_{k}$ for all $k \in \mathbb{N}, c<d$, 


$$
\lim _{k \rightarrow \infty}\left(c_{k}, d_{k}, \theta_{k}, \mu_{k}\right)=(c, d, \theta, \mu) \text { in } \mathbb{R}^{5},
$$

and

$$
\lim _{k \rightarrow \infty} f_{k}=f \quad \text { in } L^{1}(a, b)
$$

Set $\theta=\left(\theta^{-}, \theta^{+}\right)$and $\theta_{k}=\left(\theta_{k}^{-}, \theta_{k}^{+}\right)$for $k \in \mathbb{N}$ and assume that $i\left(\theta_{k}^{-}\right)=i\left(\theta_{k}^{+}\right)=$ $i\left(\theta^{-}\right)=i\left(\theta^{+}\right)$for all $k \in \mathbb{N}$. For each $k \in \mathbb{N}$ let $\left\{v_{k}\right\}_{k \in \mathbb{N}} \subset W^{2,1}(a, b)$, and assume that for any $k \in \mathbb{N}$, the function $v_{k}$ is a zeroth order solution of

$$
F\left[v_{k}\right]+\mu_{k} v_{k}+\operatorname{sgn}\left(v_{k}\right) f_{k}=0 \text { in }\left(c_{k}, d_{k}\right) \quad \text { and } \quad B\left(v_{k}, c_{k}, d_{k}\right) \in L\left(\theta_{k}\right) .
$$

Moreover, assume that $f \not \equiv 0$ in $(c, d)$. Then we have $\mu<\mu^{0}(\theta, c, d)$ if and only if

$$
\sup _{k \in \mathbb{N}}\left\|v_{k}\right\|_{W^{1, \infty}\left(c_{k}, d_{k}\right)}<\infty
$$

Furthermore, if $\mu<\mu^{0}(\theta, c, d)$, then the sequence $\left\{v_{k}\right\}$ has a convergent subsequence in $W^{2,1}(a, b)$ whose limit $v \in W^{2,1}(a, b)$ is a zeroth order solution of

$$
F[v]+\mu v+\operatorname{sgn}(v) f=0 \text { in }(c, d) \text { and } B(v, c, d)=L(\theta) .
$$

Proof By (S1), we may assume that $\theta, \theta_{k} \in(0, \pi]^{2}$, so that $v_{k}>0$ in $\left(c_{k}, d_{k}\right)$ for all $k \in \mathbb{N}$.

We first assume that (49) holds, and show that $\mu<\mu^{0}(\theta, c, d)$. By Lemma 24, $\left\{v_{k}\right\}$ has a convergent subsequence $\left\{v_{k_{j}}\right\}_{j \in \mathbb{N}}$ in $W^{2,1}(a, b)$ whose limit $v \in W^{2,1}(a, b)$ satisfies

$$
F[v]+\mu v+f=0 \text { in }(a, b) \text { and } v \geq 0 \text { in }[c, d] .
$$

Since $f \not \equiv 0$ and $F[v]+\mu v+f=0$ in $[c, d]$, we see that $v \not \equiv 0$ on $[c, d]$. By the strong maximum principle, Proposition 13 , we see that $v>0$ in $(c, d),\left(v(c), v^{\prime}(c)\right) \neq(0,0)$ and $\left(v(d), v^{\prime}(d)\right) \neq(0,0)$. Since $\left\{v_{k_{j}}\right\}_{j \in \mathbb{N}}$ converges to $v$ also in $C^{1}([a, b])$, we get $B(v, c, d)=L(\theta)$. Thus $v$ is a zeroth order solution of (50). Theorem 6 (3), with the interval $(a, b)$ replaced by $(c, d)$, assures that $\mu<\mu^{0}(\theta, c, d)$.

Next we assume that $\mu<\mu^{0}(\theta, c, d)$. We argue by contradiction, and suppose to the contrary that $\sup _{k \in \mathbb{N}}\left\|v_{k}\right\|_{W^{1, \infty}\left(c_{k}, d_{k}\right)}=\infty$. We may then assume by passing to a subsequence if necessary that $\lim _{k \rightarrow \infty}\left\|v_{k}\right\|_{W^{1, \infty}\left(c_{k}, d_{k}\right)}=\infty$. We set

$$
\tilde{v}_{k}=\left\|v_{k}\right\|_{W^{1, \infty}\left(c_{k}, d_{k}\right)}^{-1} v_{k} \quad \text { and } \quad \tilde{f}_{k}=\left\|v_{k}\right\|_{W^{1, \infty}\left(c_{k}, d_{k}\right)}^{-1} f_{k} \quad \text { on }[a, b] \quad \text { for } k \in \mathbb{N} \text {, }
$$

and observe that $\tilde{v}_{k}$ satisfy

$F\left[\tilde{v}_{k}\right]+\mu_{k} \tilde{v}_{k}+\tilde{f}_{k}=0$ in $(a, b), \quad \tilde{v}_{k}>0$ in $\left(c_{k}, d_{k}\right)$ and $B\left(\tilde{v}_{k}, c_{k}, d_{k}\right)=L\left(\theta_{k}\right)$ 
Note that $\lim _{k \rightarrow \infty} \tilde{f}_{k}=0$ in $L^{1}(a, b)$. By Lemma $24,\left\{\tilde{v}_{k}\right\}$ has a convergent subsequence $\left\{\tilde{v}_{k_{j}}\right\}_{j \in \mathbb{N}}$ in $W^{2,1}(a, b)$ whose limit $w \in W^{2,1}(a, b)$ satisfies $F[w]+$ $\mu w=0$ in $(a, b), w \geq 0$ in $[c, d]$ and $\|w\|_{W^{1, \infty}(c, d)}=1$. Since $B(w, c, d) \in$ $\overline{L(\theta)}, \mu<\mu^{0}(\theta, c, d)$ and $F\left[\varphi_{0}\right]+\mu \varphi_{0}<0=F[w]+\mu w$ in $(c, d)$, where $\varphi_{0}$ is a (positive) eigenfunction corresponding to $\mu^{0}(\theta, c, d)$, by the maximum principle, Theorem 16, we deduce that $w \leq 0$ in $(c, d)$, which implies that $w \equiv 0$ on $[a, b]$. This is a contradiction, which completes the proof.

Remark 30 We note that, in the proof above of the fact that $\mu<\mu^{0}(\theta, a, b)$ implies (49), the condition $f \not \equiv 0$ is not needed.

Proof of Theorem 6 (2) and (1) for $n=0$ In view of (S1), we may assume that $\theta \in$ $(0, \pi]^{2}$. Let $\varphi \in W^{2,1}(a, b)$ be an eigenfunction corresponding to $\mu^{0}(\theta, a, b)$.

The uniqueness of a zeroth order solution of (18) is a consequence of the comparison principle, Proposition 18, with $\psi$ replaced by $\varphi$.

We prove the existence of a zeroth order solution of (18). We treat first the case where

$$
\theta \in(0, \pi)^{2}, \quad f \in C([a, b]) \quad \text { and } \quad \min _{[a, b]} f>0 .
$$

Since $\varphi>0$ on $[a, b]$, we may choose positive constants $p_{0}<p_{1}$ so that

$$
\left(\mu^{0}(\theta, a, b)-\mu\right) p_{0} \varphi \leq f \leq\left(\mu^{0}(\theta, a, b)-\mu\right) p_{1} \varphi \quad \text { on }[a, b] .
$$

Set $\varphi_{0}=p_{0} \varphi$ and $\varphi_{1}=p_{1} \varphi$ on $[a, b]$ and note that $F\left[\varphi_{0}\right]+\mu \varphi_{0}+f \geq 0 \geq$ $F\left[\varphi_{1}\right]+\mu \varphi_{1}+f$ in $(a, b)$. Also, set $r_{0}=\varphi_{0}(a) / \sin \theta^{-}$and $r_{1}=\varphi_{1}(a) / \sin \theta^{-}$. Since $\sin \theta^{-}>0$, we have $r_{0}>0$ and $r_{1}>0$.

For each $\alpha>0$ let $v_{\alpha} \in W^{2,1}(a, b)$ be the unique solution of the initial value problem

$$
F\left[v_{\alpha}\right]+\mu v_{\alpha}+f=0 \text { in }(a, b) \quad \text { and } \quad\left(-v_{\alpha}^{\prime}(a), v_{\alpha}(a)\right)=\alpha\left(\cos \theta^{-}, \sin \theta^{-}\right) .
$$

We remark that if $v_{\alpha} \geq 0$ in $[a, b]$, then $F\left[v_{\alpha}\right]+\mu v_{\alpha}=-f \leq 0$ in $(a, b)$ and Proposition 13 yield $v_{\alpha}>0$ in $(a, b)$ and $\max \left\{v_{\alpha}(b),-v_{\alpha}^{\prime}(b)\right\}>0$. Hence, we may find a $\theta_{\alpha} \in(0, \pi]$ so that $B^{+}(v, b) \in l\left(\theta_{\alpha}\right)$.

Now we prove that $v_{\alpha}>\varphi_{1}$ in $[a, b]$ for all $\alpha>r_{1}$, in particular, $\theta_{\alpha}$ is well-defined for $\alpha \geq r_{1}$. We argue by contradiction and suppose that for some $\alpha>r_{1}$, one has $v_{\alpha}(d)=\varphi_{1}(d)$ and $v_{\alpha}>\varphi_{1}$ in $[a, d)$ due to $v_{\alpha}(a)=\alpha \sin \theta^{-}>r_{1} \sin \theta^{-}=\varphi_{1}(a)$. Since $\varphi_{1}>0$ in $[a, b]$, choose $\theta_{d, v_{\alpha}}, \theta_{d, \varphi_{1}} \in(0, \pi)$ such that $B^{+}\left(v_{\alpha}, d\right) \in l\left(\theta_{d, v_{\alpha}}\right)$ and $B^{+}\left(\varphi_{1}, d\right) \in l\left(\theta_{d, \varphi_{1}}\right)$. Moreover, it follows from $v_{\alpha}>\varphi_{1}$ in $(a, d)$ that $v_{\alpha}^{\prime}(d) \leq$ $\varphi_{1}^{\prime}(d)$, which implies $\theta_{d, \varphi_{1}} \leq \theta_{d, v_{\alpha}}$.

On the other hand, we have

$$
\begin{aligned}
& F\left[\varphi_{1}\right]+\mu \varphi_{1} \leq-f=F\left[v_{\alpha}\right]+\mu v_{\alpha} \quad \text { in }(a, d), \\
& B\left(\varphi_{1}, a, d\right) \in L\left(\theta^{-}, \theta_{d, \varphi_{1}}\right), \quad B\left(v_{\alpha}, a, d\right) \in L\left(\theta^{-}, \theta_{d, v_{\alpha}}\right) .
\end{aligned}
$$

Applying Proposition 18, we get $v_{\alpha} \leq \varphi_{1}$ in $[a, d]$, which is a contradiction. Thus $v_{\alpha}>\varphi_{1}$ in $[a, b]$ for all $\alpha>r_{1}$. 
Next, we claim that $\theta_{\alpha}<\theta^{+}$for each $\alpha>r_{1}$. In fact, if $\theta_{\alpha} \geq \theta^{+}$holds for some $\alpha>r_{1}$, arguing as above, Proposition 18 gives us a contradiction $v_{\alpha} \leq \varphi_{1}$ in $[a, b]$. Hence, $\theta_{\alpha}<\theta^{+}$for every $\alpha>r_{1}$.

We set

$$
A=\left\{\alpha>0: \theta_{\alpha}<\theta^{+} \text {and } v_{\alpha} \geq \varphi_{0} / 2 \text { on }[a, b]\right\} \quad \text { and } \quad \alpha_{0}=\inf A \text {. }
$$

The argument above shows that $\left(r_{1}, \infty\right) \subset A$ and $0 \leq \alpha_{0} \leq r_{1}$. Observe next that if $0<\alpha<r_{0} / 2$, then $v_{\alpha}(a)=\alpha \sin \theta^{-}<\left(r_{0} / 2\right) \sin \theta^{-}=\varphi_{0}(a) / 2$. Hence, we have $\left(0, r_{0} / 2\right) \cap A=\emptyset$ and $\alpha_{0} \geq r_{0} / 2$.

We now prove that

$$
\theta_{\alpha_{0}}=\theta^{+} \quad \text { and } \quad v_{\alpha_{0}}>0 \text { on }[a, b]
$$

By general ODE theory, we know that the functions $\alpha \mapsto \min _{[a, b]}\left(v_{\alpha}-\varphi_{0} / 2\right)$ and $\alpha \mapsto \theta_{\alpha}$ are continuous on $(0, \infty)$ and on $\left\{\alpha \in(0, \infty): v_{\alpha} \geq 0\right.$ in $\left.[a, b]\right\}$, respectively. By the definition of $\alpha_{0}$ together with the continuity mentioned above, we have $v_{\alpha_{0}} \geq \varphi_{0} / 2$ on $[a, b]$ and $\theta_{\alpha_{0}} \leq \theta^{+}$. Moreover, if $\theta_{\alpha_{0}}<\theta^{+}$, then, by applying Proposition 18 , we get $v_{\alpha_{0}} \geq \varphi_{0}$ on $[a, b]$, which implies that, for some $\alpha_{1} \in\left(0, \alpha_{0}\right), \theta_{\alpha_{1}}<\theta^{+}$and $v_{\alpha_{1}} \geq \varphi_{0} / 2$ on $[a, b]$, and hence, by the definition of $\alpha_{0}, \alpha_{0} \leq \alpha_{1}$, a contradiction. Thus, we find that $\theta_{\alpha_{0}}=\theta^{+}$and $v_{\alpha_{0}} \geq \varphi_{0} / 2>0$ on $[a, b]$.

The function $v_{\alpha_{0}}$ is a zeroth order solution of (18), and the proof of existence is done under the additional hypotheses (51).

To remove the extra condition (51), given $f \in L^{1}(a, b)$, with $f \geq 0$ in $(a, b)$, and $\theta \in(0, \pi]^{2}$, we select sequences $\left\{f_{k}\right\}_{k \in \mathbb{N}} \subset C([a, b])$ and $\left\{\theta_{k}\right\}_{k \in \mathbb{N}} \subset(0, \pi)^{2}$, so that $\min _{[a, b]} f_{k}>0$ for all $k \in \mathbb{N}$.

Thanks to Lemma 26, we have the convergence

$$
\lim _{k \rightarrow \infty} \mu^{0}\left(\theta_{k}, a, b\right)=\mu^{0}(\theta, a, b) .
$$

We may therefore assume that $\mu<\mu^{0}\left(\theta_{k}, a, b\right)$ for all $k \in \mathbb{N}$. The previous argument ensures that for each $k \in \mathbb{N}$ there is a zeroth order solution $v_{k} \in W^{2,1}(a, b)$ of (18), with $f_{k}$ and $\theta_{k}$ in place of $f$ and $\theta$, respectively. We apply Lemma 29, to conclude that there exists a zeroth order solution of (18). This completes the proof.

We give some definitions and observations needed for the proof of claim (1) of Theorem 6.

Fix any admissible $(n, \theta)$ and $\mu \in\left(-\infty, \mu^{n}(\theta, a, b)\right)$. Let $\varphi_{n}$ be an eigenfunction corresponding to $\mu^{n}(\theta, a, b)$ and $\left\{x_{i}\right\}_{i=1}^{n}$ be the increasing sequence of zeroes of $\varphi_{n}$ in $(a, b)$. We set $x_{0}=a$ and $x_{n+1}=b$. We set $\theta_{1}^{-}=\theta^{-}$and $\theta_{n+1}^{+}=\theta^{+}$, choose two sequences $\left\{\theta_{i}^{-}\right\}_{i=2}^{n+1},\left\{\theta_{i}^{+}\right\}_{i=1}^{n} \subset\{\pi, 2 \pi\}$ so that $B\left(\varphi_{n}, x_{i-1}, x_{i}\right) \in L\left(\theta_{i}^{-}, \theta_{i}^{+}\right)$ for all $i \in\{1, \ldots, n+1\}$, and set $\theta_{i}=\left(\theta_{i}^{-}, \theta_{i}^{+}\right)$for $i \in\{1, \ldots, n+1\}$. Note that $\mu^{0}\left(\theta_{i}, x_{i-1}, x_{i}\right)=\mu^{n}(\theta, a, b)$ for all $i \in\{1, \ldots, n+1\}$.

Let $M>0$ and $c, d \in[a, b]$ be such that $c \leq d$. Fix any $i \in\{1, \ldots, n+1\}$. Let $u_{i} \in W^{2,1}(c, d)$ be a zeroth order solution of (18), with $\theta, a$ and $b$ replaced 
by $\theta_{i}, c$ and $d$, respectively, provided $c<d$ and $\mu<\mu^{0}\left(\theta_{i}, c, d\right)$. The existence and uniqueness of $u_{i}$ is assured by the claims (2) and (1) for $n=0$ of Theorem 6 , which have already been proved above. We define $\Phi_{M}^{-}(c, d, i), \Phi_{M}^{+}(c, d, i) \in \mathbb{R}$, respectively, by

$$
\Phi_{M}^{-}(c, d, i)= \begin{cases}0 & \text { if } c=d, \\ \min \left\{\left|u_{i}(c)\right|+\left|u_{i}^{\prime}(c)\right|, M\right\} & \text { if } c<d \text { and } \mu<\mu^{0}\left(\theta_{i}, c, d\right), \\ M & \text { otherwise, }\end{cases}
$$

and

$$
\Phi_{M}^{+}(c, d, i)= \begin{cases}0 & \text { if } c=d \\ \min \left\{\left|u_{i}(d)\right|+\left|u_{i}^{\prime}(d)\right|, M\right\} & \text { if } c<d \text { and } \mu<\mu^{0}\left(\theta_{i}, c, d\right) \\ M & \text { otherwise }\end{cases}
$$

Similarly, we write

$$
\Phi^{-}(c, d, i)= \begin{cases}0 & \text { if } c=d \\ \left|u_{i}(c)\right|+\left|u_{i}^{\prime}(c)\right| & \text { if } c<d \text { and } \mu<\mu^{0}\left(\theta_{i}, c, d\right)\end{cases}
$$

and

$$
\Phi^{+}(c, d, i)= \begin{cases}0 & \text { if } c=d, \\ \left|u_{i}(d)\right|+\left|u_{i}^{\prime}(d)\right| & \text { if } c<d \text { and } \mu<\mu^{0}\left(\theta_{i}, c, d\right) .\end{cases}
$$

In the definition above we note that for any $i \in\{2, \ldots, n+1\}$, if $c<d$ and $\mu<\mu^{0}\left(\theta_{i}, c, d\right)$, then $u_{i}(c)=0$ and $\Phi_{M}^{-}(c, d, i)=\min \left\{M,\left|u_{i}^{\prime}(c)\right|\right\}>0$, that for any $i \in\{1, \ldots, n\}$, if $c<d$ and $\mu<\mu^{0}\left(\theta_{i}, c, d\right)$, then $u_{i}(d)=0$ and $\Phi_{M}^{+}(c, d, i)=$ $\min \left\{M,\left|u_{i}^{\prime}(d)\right|\right\}>0$. Note also that for any $i \in\{1, \ldots, n+1\}$ and $C>0$, we have

$$
\begin{aligned}
& \min \left\{\Phi_{M}^{-}(c, d, i), C\right\}=\Phi_{\min \{M, C\}}^{-}(c, d, i) \quad \text { and } \\
& \min \left\{\Phi_{M}^{+}(c, d, i), C\right\}=\Phi_{\min \{M, C\}}^{+}(c, d, i) .
\end{aligned}
$$

Lemma 31 Let $M>0, \mu \in \mathbb{R}$ and $\left\{\theta_{i}\right\}_{i=1}^{n+1}$ be as above. Let $f \in C([a, b])$ satisfy $f>0$ on $[a, b]$. For any $i \in\{1, \ldots, n+1\}$ the functions $(c, d) \mapsto \Phi_{M}^{ \pm}(c, d, i)$ are continuous on the set $\left\{(x, y) \in[a, b]^{2}: x \leq y\right\}$.

Proof Set $\Delta=\left\{(x, y) \in[a, b]^{2}: x \leq y\right\}$. Fix any convergent sequence $\left\{\left(c_{k}, d_{k}\right)\right\}_{k \in \mathbb{N}}$ of points in $\Delta$. Set $(c, d)=\lim _{k \rightarrow \infty}\left(c_{k}, d_{k}\right)$. We need to prove that

$$
\lim _{k \rightarrow \infty} \Phi_{M}^{ \pm}\left(c_{k}, d_{k}, i\right)=\Phi_{M}^{ \pm}(c, d, i)
$$


In the proof which follows, for each $k \in \mathbb{N}, u_{i, k} \in W^{2,1}\left(c_{k}, d_{k}\right)$ denotes the unique zeroth order solution of (18), with $\theta, a$ and $b$ replaced by $\theta_{i}, c_{k}$ and $d_{k}$, respectively, provided $a \leq c_{k}<d_{k} \leq b$ and $\mu<\mu^{0}\left(\theta_{i}, c_{k}, d_{k}\right)$.

We consider first the case when $c=d$. By definition, we have $\Phi_{M}^{ \pm}(c, d, i)=0$. To prove (52), we suppose to the contrary that either

$$
\limsup _{k \rightarrow \infty} \Phi_{M}^{-}\left(c_{k}, d_{k}, i\right)>0 \quad \text { or } \quad \limsup _{k \rightarrow \infty} \Phi_{M}^{+}\left(c_{k}, d_{k}, i\right)>0
$$

We may choose a subsequence $\left\{\left(c_{k_{j}}, d_{k_{j}}\right)\right\}_{j \in \mathbb{N}}$ of $\left\{\left(c_{k}, d_{k}\right)\right\}$ so that

$$
\begin{aligned}
& \lim _{j \rightarrow \infty} \Phi_{M}^{-}\left(c_{k_{j}}, d_{k_{j}}, i\right)=\underset{k \rightarrow \infty}{\limsup } \Phi_{M}^{-}\left(c_{k}, d_{k}, i\right) \quad \text { and } \\
& \lim _{j \rightarrow \infty} \Phi_{M}^{+}\left(c_{k_{j}}, d_{k_{j}}, i\right)=\underset{k \rightarrow \infty}{\limsup } \Phi_{M}^{+}\left(c_{k}, d_{k}, i\right) .
\end{aligned}
$$

Obviously, we may assume by passing again to a subsequence if necessary that $c_{k_{j}}<d_{k_{j}}$ and $d_{k_{j}}-c_{k_{j}}<b-a$ for all $j \in \mathbb{N}$. By Lemma 28, we have $\lim _{j \rightarrow \infty} \mu^{0}\left(\theta_{i}, c_{k_{j}}, d_{k_{j}}\right)=\infty$. Hence, we may moreover assume that $\mu<$ $\mu^{0}\left(\theta_{i}, c_{k_{j}}, d_{k_{j}}\right)$ for all $j \in \mathbb{N}$. Noting by our choice of $\theta_{i}$ that either $u_{i, k_{j}}\left(c_{k_{j}}\right)=0$ or $u_{i, k_{j}}\left(d_{k_{j}}\right)=0$ for all $j \in \mathbb{N}$ and that $d_{k_{j}}-c_{k_{j}} \rightarrow 0$, we apply Lemma 23 , to obtain

$$
\lim _{j \rightarrow \infty}\left\|u_{i, k_{j}}\right\|_{W^{1, \infty}\left(c_{k_{j}}, d_{k_{j}}\right)}=0,
$$

which readily yields $\lim _{j \rightarrow \infty} \Phi_{M}^{-}\left(c_{k_{j}}, d_{k_{j}}, i\right)=\lim _{j \rightarrow \infty} \Phi_{M}^{+}\left(c_{k_{j}}, d_{k_{j}}, i\right)=0$. This contradicts (53), which proves (52).

What remains is the case where $c<d$. We may assume by replacing $\left\{\left(c_{k}, d_{k}\right)\right\}$ by $\left\{\left(c_{k+K}, d_{k+K}\right)\right\}_{k \in \mathbb{N}}$, with $K \in \mathbb{N}$ sufficiently large if necessary, that $c_{k}<d_{k}$ for all $k \in \mathbb{N}$.

Now we consider the case where $\mu<\mu^{0}\left(\theta_{i}, c, d\right)$. In view of the continuity of the eigenvalues, Lemma 26, we may now assume that $\mu<\mu^{0}\left(\theta_{i}, c_{k}, d_{k}\right)$ for all $k \in \mathbb{N}$. We denote by $u_{i}$ the zeroth order solution of (18), with $\theta, a$ and $b$, replaced by $\theta_{i}, c$ and $d$, respectively. By Lemma 29 , we deduce that $\lim _{k \rightarrow \infty} u_{i, k}=u_{i}$ in $C^{1}([a, b])$, which, in particular, ensures that (52) holds.

Next we consider the case where $c<d$ and $\mu \geq \mu^{0}\left(\theta_{i}, c, d\right)$. Note that $\Phi_{M}^{-}(c, d, i)=\Phi_{M}^{+}(c, d, i)=M$. If $\mu>\mu^{0}\left(\theta_{i}, c, d\right)$, then, by the continuity of the eigenvalues, we have $\mu>\mu^{0}\left(\theta_{i}, c_{k}, d_{k}\right)$ and hence $\Phi_{M}^{-}\left(c_{k}, d_{k}, i\right)=\Phi_{M}^{+}\left(c_{k}, d_{k}, i\right)=$ $M$ for sufficiently large $k \in \mathbb{N}$, and (52) follows. We may therefore assume that $\mu=\mu^{0}\left(\theta_{i}, c, d\right)$. To prove (52), we argue by contradiction, and suppose that

$$
\liminf _{k \rightarrow \infty} \Phi_{M}^{-}\left(c_{k}, d_{k}, i\right)<M \quad \text { or } \quad \liminf _{k \rightarrow \infty} \Phi_{M}^{+}\left(c_{k}, d_{k}, i\right)<M
$$

This, in particular, implies that $\mu<\mu^{0}\left(\theta_{i}, c_{k}, d_{k}\right)$ for sufficiently large $k \in \mathbb{N}$. We may select a subsequence $\left\{\left(c_{k_{j}}, d_{k_{j}}\right)\right\}_{j \in \mathbb{N}}$ of $\left\{\left(c_{k}, d_{k}\right)\right\}$ so that $\mu<\mu^{0}\left(\theta_{i}, c_{k_{j}}, d_{k_{j}}\right)$ for all $j \in \mathbb{N}$, 


$$
\lim _{j \rightarrow \infty} \Phi_{M}^{-}\left(c_{k_{j}}, d_{k_{j}}, i\right)=\liminf _{k \rightarrow \infty} \Phi_{M}^{-}\left(c_{k}, d_{k}, i\right)
$$

and

$$
\lim _{j \rightarrow \infty} \Phi_{M}^{+}\left(c_{k_{j}}, d_{k_{j}}, i\right)=\liminf _{k \rightarrow \infty} \Phi_{M}^{+}\left(c_{k}, d_{k}, i\right)
$$

Since $\lim _{j \rightarrow \infty} \mu^{0}\left(\theta_{i}, c_{k_{j}}, d_{k_{j}}\right)=\mu$, by applying Lemma 29 together with Lemma 24, we see that $\sup _{j \in \mathbb{N}} \min _{[a, b]}\left(\left|u_{i, k_{j}}\right|+\left|u_{i, k_{j}}^{\prime}\right|\right)=\infty$, which yields

$$
\lim _{j \rightarrow \infty} \Phi_{M}^{-}\left(c_{k_{j}}, d_{k_{j}}, i\right)=\liminf _{j \rightarrow \infty} \Phi_{M}^{+}\left(c_{k_{j}}, d_{k_{j}}, i\right)=M
$$

This contradicts (54), which proves (52). The proof is complete.

Proof of Theorem 6 (1) The claim (1) for $n=0$ has already been proved.

We assume for the moment that $f \in C([a, b])$ with $f>0$ in $[a, b]$, and prove the existence of an $n$th order solution of (18).

Set

$$
\Delta_{n}=\left\{y=\left(y_{1}, \ldots, y_{n}\right) \in \mathbb{R}^{n}: y_{i}>0 \text { for all } i \in\{1, \ldots, n\}, \sum_{i=1}^{n} y_{i}<b-a\right\} .
$$

Given $y=\left(y_{1}, \ldots, y_{n}\right) \in \mathbb{R}^{n}$, we write $y_{n+1}=b-a-\sum_{i=1}^{n} y_{i}$ and note that $\bar{\Delta}_{n}=\left\{y=\left(y_{1}, \ldots, y_{n}\right) \in \mathbb{R}^{n}: y_{i} \geq 0\right.$ for all $\left.i \in\{1, \ldots, n\}, 0 \leq y_{n+1} \leq b-a\right\}$.

Fix any $M>0$ and define a mapping $T_{M}: \bar{\Delta}_{n} \rightarrow \mathbb{R}^{n}$ by

$$
T_{M}(y)=\left(T_{M, 1}(y), \ldots, T_{M, n}(y)\right),
$$

where $T_{M, i}(y)=\Phi_{M}^{+}\left(z_{i-1}, z_{i}, i\right)-\Phi_{M}^{-}\left(z_{i}, z_{i+1}, i+1\right)$ for $i \in\{1, \ldots, n\}, z_{0}=a$ and $z_{i}=a+\sum_{j=1}^{i} y_{j}$ for $i \in\{1, \ldots, n+1\}$.

Note by the assumption that $f \in C([a, b])$ and $f>0$ in $[a, b]$ and by Lemma 31 that $T_{n}: \bar{\Delta}_{n} \rightarrow \mathbb{R}^{n}$ is a continuous mapping.

We show that $T_{M}$ has a zero in $\Delta_{n}$, and for this, we consider the degree, $\operatorname{deg}\left(T_{M}, 0, \Delta_{n}\right)$, of $T_{M}$ on $\Delta_{n}$ and prove that $\operatorname{deg}\left(T_{M}, 0, \Delta_{n}\right)=1$.

Observe that $\partial \Delta_{n}=\Gamma_{n, 0} \cup \Gamma_{n, 1}$, where $\Gamma_{n, 0}=\left\{y \in \bar{\Delta}_{n}: y_{n+1}>0, y_{i}=\right.$ 0 for some $i \in\{1, \ldots, n\}\}$ and $\Gamma_{n, 1}=\left\{y \in \bar{\Delta}_{n}: y_{n+1}=0\right\}$.

Let $y \in \Gamma_{n, 1}$, note that $\sum_{i=1}^{n} y_{i}=b-a>0$ and set $j=\max \{i \in\{1, \ldots, n\}$ : $\left.y_{i} \neq 0\right\}$. Since $y_{j}>0$ and $y_{j+1}=0$, we have

$$
\Phi_{M}^{+}\left(z_{j-1}, z_{j}, j\right)>0, \quad \Phi_{M}^{-}\left(z_{j}, z_{j+1}, j+1\right)=0 \quad \text { and } \quad T_{M, j}(y)>0 .
$$

Observe moreover that for any $t \in[0,1]$,

$$
(1-t) T_{M, j}(y)+t y_{j}>0
$$


which shows, together with the continuity of $T_{M}$ and the compactness of $\Gamma_{n, 1}$, that

$$
\min \left\{\left|(1-t) T_{M}(y)+t y\right|: y \in \Gamma_{n, 1}, t \in[0,1]\right\}>0 .
$$

Hence, fixing a point $p=\left(p_{1}, \ldots, p_{n}\right) \in \Delta_{n}$, we may choose $\delta \in(0,1)$ so that

$$
(1-t) T_{M}(y)+t(y-\delta p) \neq 0 \quad \text { for all }(y, t) \in \Gamma_{n, 1} \times[0,1]
$$

Next let $y \in \Gamma_{n, 0}$. Set $j=\max \left\{i \in\{1, \ldots, n\}: y_{i}=0\right\}$ and note that $y_{j}=0$ and $y_{j+1}>0$. Accordingly, we have

$$
\Phi_{M}^{+}\left(z_{j-1}, z_{j}, j\right)=0, \quad \Phi_{M}^{-}\left(z_{j}, z_{j+1}, j+1\right)>0 \quad \text { and } \quad T_{M, j}(y)<0 .
$$

Note here that for any $t \in[0,1]$,

$$
(1-t) T_{M, j}(y)+t\left(y_{j}-\delta p_{j}\right)=(1-t) T_{M, j}(y)-\delta t p_{j}<0,
$$

which shows that

$$
(1-t) T_{M}(y)+t(y-\delta p) \neq 0 \quad \text { for all }(y, t) \in \Gamma_{n, 0} \times[0,1] .
$$

This together with (55) ensures that

$$
(1-t) T_{M}(y)+t(y-\delta p) \neq 0 \quad \text { for all }(y, t) \in \partial \Delta_{n} \times[0,1] .
$$

Hence, by the homotopy invariance of degree, we have $\operatorname{deg}\left(T_{M}, 0, \Delta_{n}\right)$ $=\operatorname{deg}\left(h, 0, \Delta_{n}\right)$, where $h$ is the function on $\mathbb{R}^{n}$ defined by $h(y)=y-\delta p$. Thus, noting that $h$ vanishes exactly at the point $\delta p \in \Delta_{n}$ and that $\delta p$ is a regular value of $h$, we see that $\operatorname{deg}\left(h, 0, \Delta_{n}\right)=1$ and conclude that $\operatorname{deg}\left(T_{M}, 0, \Delta_{n}\right)=1$.

The fact that $\operatorname{deg}\left(T_{M}, 0, \Delta_{n}\right)=1$ guarantees that there exists a zero $y_{M} \in \Delta_{n}$ of $T_{M}$.

We intend to show that there is a constant $M>0$ such that

$$
\begin{array}{ll}
\Phi_{M}^{-}\left(z_{M, i-1}, z_{M, i}, i\right)<M & \text { and } \\
\Phi_{M}^{+}\left(z_{M, i-1}, z_{M, i}, i\right)<M & \text { for all } i \in\{1, \ldots, n+1\},
\end{array}
$$

where

$$
z_{M, 0}=a, \quad z_{M, i}=a+\sum_{j=1}^{i} y_{M, j} \text { for } i \in\{1, \ldots, n\} \quad \text { and } z_{M, n+1}=b
$$

For any $M>0$, let $y_{M}=\left(y_{M, 1}, \ldots, y_{M, n}\right) \in \Delta_{n}$ be a zero of $T_{M}$ and define the sequence $\left\{z_{M}, i\right\}_{i=0}^{n+1}$ by (57). We select a sequence $\left\{M_{k}\right\}_{k \in \mathbb{N}} \subset(0, \infty)$ so that $\lim _{k \rightarrow \infty} M_{k}=\infty$ and $\lim _{k \rightarrow \infty} z_{M_{k}}=z$, where $z=\left(z_{0}, z_{1}, \ldots, z_{n}, z_{n+1}\right) \in \mathbb{R}^{n+2}$ satisfies $a=z_{0} \leq z_{1} \leq \cdots \leq z_{n+1}=b$. 
The first step is to show that if either $z_{i-1}=z_{i}$, or $z_{i-1}<z_{i}$ and $\mu<$ $\mu^{0}\left(\theta_{i}, z_{i-1}, z_{i}\right)$ for some $i \in\{1, \ldots, n+1\}$, then, for sufficiently large $k \in \mathbb{N}$,

$$
\Phi_{M_{k}}^{-}\left(z_{M_{k}, i-1}, z_{M_{k}, i}, i\right)<M_{k} \quad \text { and } \quad \Phi_{M_{k}}^{+}\left(z_{M_{k}, i-1}, z_{M_{k}, i}, i\right)<M_{k} .
$$

To see this, assume that either $z_{i-1}=z_{i}$, or $z_{i-1}<z_{i}$ and $\mu<\mu^{0}\left(\theta_{i}, z_{i-1}, z_{i}\right)$ for some $i \in\{1, \ldots, n+1\}$.

Since $\lim _{k \rightarrow \infty} M_{k}=\infty$, by Lemma 31, we get for any $M>\Phi^{-}\left(z_{i-1}, z_{i}, i\right)$,

$$
\begin{aligned}
\lim _{k \rightarrow \infty} \min \left\{M, \Phi_{M_{k}}^{-}\left(z_{M_{k}, i-1}, z_{M_{k}, i}, i\right)\right\} & =\lim _{k \rightarrow \infty} \Phi_{M}^{-}\left(z_{M_{k}, i-1}, z_{M_{k}, i}, i\right) \\
& =\Phi_{M}^{-}\left(z_{i-1}, z_{i}, i\right)=\Phi^{-}\left(z_{i-1}, z_{i}, i\right)
\end{aligned}
$$

which implies that

$$
\lim _{k \rightarrow \infty} \Phi_{M_{k}}^{-}\left(z_{M_{k}, i-1}, z_{M_{k}, i}, i\right)=\Phi^{-}\left(z_{i-1}, z_{i}, i\right)
$$

Similarly, we get

$$
\lim _{k \rightarrow \infty} \Phi_{M_{k}}^{+}\left(z_{M_{k}, i-1}, z_{M_{k}, i}, i\right)=\Phi^{+}\left(z_{i-1}, z_{i}, i\right)
$$

Thus, for sufficiently large $k \in \mathbb{N}$, we have

$$
\Phi_{M_{k}}^{-}\left(z_{M_{k}, i-1}, z_{M_{k}, i}, i\right)<M_{k} \text { and } \Phi_{M_{k}}^{+}\left(z_{M_{k}, i-1}, z_{M_{k}, i}, i\right)<M_{k}
$$

Next we show that there exists $i \in\{1, \ldots, n+1\}$ such that either

$$
z_{i-1}=z_{i}, \quad \text { or } \quad z_{i-1}<z_{i} \text { and } \mu<\mu^{0}\left(\theta_{i}, z_{i-1}, z_{i}\right) \text {. }
$$

Recalling the definition of $\left\{x_{i}\right\}_{i=0}^{n+1}$, we set $j=\min \left\{i \in\{1, \ldots, n+1\}: z_{i} \leq x_{i}\right\}$, and note that the inclusion, $\left(z_{j-1}, z_{j}\right) \subset\left(x_{j-1}, x_{j}\right)$, holds.

If $z_{j-1}=z_{j}$, then we have nothing to prove. We may thus assume that $z_{j-1}<z_{j}$. Noting that $z_{0}=a, z_{n+1}=b, \varphi_{n}\left(x_{i}\right)=0$ for all $i \in\{1, \ldots, n\}$, and $\left|\varphi_{n}\right|>$ 0 in $\left(x_{i-1}, x_{i}\right)$ for all $i \in\{1, \ldots, n+1\}$, the function $\varphi_{n}$ can be regarded as a zeroth order eigenfunction of the problem, $F[\varphi]+\mu^{n}(\theta, a, b) \varphi=0$ in $\left(z_{j-1}, z_{j}\right)$ and $B\left(\varphi, z_{j-1}, z_{j}\right) \in L(\tau)$, for some $\tau \in(0,2 \pi]^{2}$ satisfying the order relation, $(0, \tau) \leq\left(0, \theta_{j}\right)$. By Theorem 4 , we see that $\mu^{0}\left(\tau, z_{j-1}, z_{j}\right) \leq \mu^{0}\left(\theta_{j}, z_{j-1}, z_{j}\right)$. It is obvious that $\mu^{0}\left(\tau, z_{j-1}, z_{j}\right)=\mu^{n}(\theta, a, b)$. Hence, we have $\mu<\mu^{0}\left(\tau, z_{j-1}, z_{j}\right) \leq$ $\mu^{0}\left(\theta_{j}, z_{j-1}, z_{j}\right)$.

Now we prove that (59) holds for all $i \in\{1, \ldots, n+1\}$. It is enough to show that for any $i, j \in\{1, \ldots, n+1\}$, if $|i-j|=1$ and (59) holds for this $i$, then (59), with $j$ in place of $i$, holds. Fix any $i, j \in\{1, \ldots, n+1\}$ so that $|i-j|=1$ and (59) holds for $i$. According to (58), choosing $k \in \mathbb{N}$ sufficiently large, we have

$$
\Phi_{M_{k}}^{-}\left(z_{M_{k}, i-1}, z_{M_{k}, i}, i\right)<M_{k} \quad \text { and } \quad \Phi_{M_{k}}^{+}\left(z_{M_{k}, i-1}, z_{M_{k}, i}, i\right)<M_{k}
$$


Since $T_{M_{k}}\left(y_{M_{k}}\right)=0$, the inequalities above yield

$$
\Phi_{M_{k}}^{+}\left(z_{M_{k}, j-1}, z_{M_{k}, j}, j\right)=\Phi_{M_{k}}^{-}\left(z_{M_{k}, i-1}, z_{M_{k}, i}, i\right)<M_{k} \quad \text { if } j=i-1
$$

and

$$
\Phi_{M_{k}}^{-}\left(z_{M_{k}, j-1}, z_{M_{k}, j}, j\right)=\Phi_{M_{k}}^{+}\left(z_{M_{k}, i-1}, z_{M_{k}, i}, i\right)<M_{k} \quad \text { if } j=i+1,
$$

which implies that (59), with $j$ in place of $i$, holds.

Thus, (59) holds for all $i \in\{1, \ldots, n+1\}$ and, consequently, (56) holds for some $M>0$.

We now fix $M>0$ so that (56) holds. Since $y_{M} \in \Delta_{n}$, we have $z_{M, i-1}<$ $z_{M, i}$ for all $i \in\{1, \ldots, n+1\}$. By (56), we get $\mu<\mu^{0}\left(z_{M, i-1}, z_{M, i}, i\right)$ for all $i \in\{1, \ldots, n+1\}$, and moreover, since $T_{M}\left(y_{M}\right)=0$ and $\Phi_{M}^{ \pm}\left(z_{M, i-1}, z_{M, i}, i\right)=$ $\Phi^{ \pm}\left(z_{M, i-1}, z_{M, i}, i\right)$ for every $i \in\{1, \ldots, n+1\}$,

$$
\Phi^{+}\left(z_{M, i-1}, z_{M, i}, i\right)=\Phi^{-}\left(z_{M, i}, z_{M, i+1}, i+1\right) \quad \text { for all } i \in\{1, \ldots, n\}
$$

Consequently, for any $i \in\{1, \ldots, n+1\}$, there exists a zeroth order solution $u_{i} \in$ $W^{2,1}\left(z_{M, i-1}, z_{M, i}\right)$ of

$F\left[u_{i}\right]+\mu u_{i}+\operatorname{sgn}\left(u_{i}\right) f=0$ in $\left(z_{M, i-1}, z_{M, i}\right) \quad$ and $\quad B\left(u_{i}, z_{M, i-1}, z_{M, i}\right) \in L\left(\theta_{i}\right)$,

and moreover, thanks to (60)

$$
u_{i}^{\prime}\left(z_{M, i}\right)=u_{i+1}^{\prime}\left(z_{M, i}\right) \quad \text { for all } i \in\{1, \ldots, n\}
$$

If we define $u \in W^{2,1}(a, b)$ by setting

$$
u(x)=u_{i}(x) \text { for } x \in\left[z_{M, i-1}, z_{M, i}\right] \text { and } i \in\{1, \ldots, n+1\},
$$

then $u$ is an $n$th order solution of (18).

Finally, we remove the additional assumption that $f \in C([a, b])$ and $f>0$ in $[a, b]$, and assume just that $f \geq 0$ and $f \neq \equiv 0$ in $(a, b)$. We introduce a sequence $\left\{f_{k}\right\}_{k \in \mathbb{N}} \subset C([a, b])$ such that $f_{k}>0$ on $[a, b]$ and $\lim _{k \rightarrow \infty} f_{k}=f$ in $L^{1}(a, b)$. For $k \in \mathbb{N}$ let $w_{k} \in W^{2,1}(a, b)$ be an $n$th order solution of (18), with $f$ replaced by $f_{k}$, and let $\left\{\xi_{k, i}\right\}_{i=1}^{n}$ be the increasing sequence of zeroes of $w_{k}$. We set $\xi_{k, 0}=a$ and $\xi_{k, n+1}=b$, and we may assume by taking a subsequence of $\left\{f_{k}\right\}$ if necessary that $\lim _{k \rightarrow \infty}\left(\xi_{k, 0}, \ldots, \xi_{k, n+1}\right)=\left(\eta_{0}, \ldots, \eta_{n+1}\right)$ in $\mathbb{R}^{n+2}$ for some $\left(\eta_{0}, \ldots, \eta_{n+1}\right) \in$ $\mathbb{R}^{n+2}$ such that $a=\eta_{0} \leq \eta_{1} \leq \cdots \leq \eta_{n+1}=b$. Moreover, since either $w_{k}>0$ in $\left(\xi_{k, i}, \xi_{k, i+1}\right)$ for all $k$ or else $w_{k}<0$ in $\left(\xi_{k, i}, \xi_{k, i+1}\right)$ for all $k$, it is not difficult to check that

$$
\operatorname{sgn}\left(w_{k}\right) f_{k} \rightarrow f \quad\left(\text { resp. } \quad \operatorname{sgn}\left(w_{k}\right) f_{k} \rightarrow-f\right) \quad \text { strongly in } L^{1}\left(\eta_{i}, \eta_{i+1}\right)
$$


provided $w_{k}>0$ [resp. $w_{k}<0$ ] in $\left(\xi_{k, i}, \xi_{k, i+1}\right)$ and $\eta_{i+1}-\eta_{i}>0$. Therefore, we have either

$$
\begin{aligned}
& \operatorname{sgn}\left(w_{k}\right) f_{k} \rightarrow g=\sum_{i=0}^{n}(-1)^{i} \chi_{\left(\eta_{i}, \eta_{i+1}\right)} f \text { or } \\
& \operatorname{sgn}\left(w_{k}\right) f_{k} \rightarrow g=\sum_{i=0}^{n}(-1)^{i+1} \chi_{\left(\eta_{i}, \eta_{i+1}\right)} f
\end{aligned}
$$

strongly in $L^{1}(a, b)$ where $\chi_{I}$ denotes the characteristic function of $I \subset \mathbb{R}$. Remark that $|f|=|g|$ in $(a, b)$.

We set $j=\min \left\{i \in\{1, \ldots, n+1\}: \eta_{i} \leq x_{i}\right\}$, and as before, we have either $\eta_{j-1}=\eta_{j}$ or $\mu<\mu^{0}\left(\theta_{j}, x_{j-1}, x_{j}\right) \leq \mu^{0}\left(\theta_{j}, \eta_{j-1}, \eta_{j}\right)$. Using Lemmas 29 and 24 and Remark 30, we deduce that $\left\{w_{k}\right\}$ has a convergent subsequence $\left\{w_{k_{\ell}}\right\}_{\ell \in \mathbb{N}}$ in $W^{2,1}(a, b)$ and the limit $w:=\lim _{\ell \rightarrow \infty} w_{k_{\ell}}$ is a solution of $F[w]+\mu w+g=0$ in $(a, b)$.

Fix any $i \in\{1, \ldots, n+1\}$, and observe that if $\eta_{i-1}=\eta_{i}$, then $B^{-}\left(w, \eta_{i-1}\right) \in$ $\overline{l\left(\theta_{i}^{-}\right)}, B^{+}\left(w, \eta_{i}\right) \in \overline{l\left(\theta_{i}^{+}\right)}$and, hence, $w\left(\eta_{i}\right)=w^{\prime}\left(\eta_{i}\right)=0$ due to the fact that $\left(0, \theta_{i}\right)$ is admissible. Observe by the strong maximum principle, Proposition 13, that if $\eta_{i-1}<\eta_{i}$, then $w \equiv 0$ on $\left[\eta_{i-1}, \eta_{i}\right], w>0$ in $\left(\eta_{i-1}, \eta_{i}\right)$ or $w<0$ in $\left(\eta_{i-1}, \eta_{i}\right)$. Moreover, in the case where $|w|>0$ in $\left(\eta_{i-1}, \eta_{i}\right)$, we have $\left|w\left(\eta_{i-1}\right)\right|+\left|w^{\prime}\left(\eta_{i-1}\right)\right|>0$ and $\left|w\left(\eta_{i}\right)\right|+\left|w^{\prime}\left(\eta_{i}\right)\right|>0$. Also, we have $w\left(\eta_{i}\right)=0$ for all $i \in\{1, \ldots, n\}$. Note here by the continuity of $w$ that the condition, $|w|>0$ in $\left(\eta_{i-1}, \eta_{i}\right)$, is equivalent to stating that either $w>0$ in $\left(\eta_{i-1}, \eta_{i}\right)$ or $w<0$ in $\left(\eta_{i-1}, \eta_{i}\right)$.

Since $f \not \equiv 0$ in $(a, b)$, there exists an $i \in\{1, \ldots, n\}$ such that $\eta_{i-1}<\eta_{i}$ and $f \not \equiv 0$ in $\left(\eta_{i-1}, \eta_{i}\right)$, which implies that $|w|>0$ in $\left(\eta_{i-1}, \eta_{i}\right),\left|w\left(\eta_{i-1}\right)\right|+\left|w^{\prime}\left(\eta_{i-1}\right)\right|>0$ and $\left|w\left(\eta_{i}\right)\right|+\left|w^{\prime}\left(\eta_{i}\right)\right|>0$. Because of the $C^{1}$-regularity of $w$, we see that if $j \in$ $\{1, \ldots, n+1\}$ and $|j-i|=1$, then $\eta_{j-1}<\eta_{j},\left|w\left(\eta_{j-1}\right)\right|+\left|w^{\prime}\left(\eta_{j-1}\right)\right|>0$ and $\left|w\left(\eta_{j}\right)\right|+\left|w^{\prime}\left(\eta_{j}\right)\right|>0$. This shows that for all $i \in\{1, \ldots, n+1\}$, we have $\eta_{i-1}<$ $\eta_{i},|w|>0$ in $\left(\eta_{i-1}, \eta_{i}\right),\left|w\left(\eta_{i-1}\right)\right|+\left|w^{\prime}\left(\eta_{i-1}\right)\right|>0$ and $\left|w\left(\eta_{i}\right)\right|+\left|w^{\prime}\left(\eta_{i}\right)\right|>0$. Thus we conclude that $g=\lim _{k \rightarrow \infty} \operatorname{sgn}\left(w_{k}\right) f_{k}=\operatorname{sgn}(w) f$ in $L^{1}(a, b)$ and $w$ is an $n$th order solution of (18).

\section{Preliminary observations in the radial case}

This section provides some preliminaries for the proof of main results in the radial case.

Let $a \in[0, R)$ and $q \in[1, \infty]$. We denote by $L_{\mathrm{r}}^{q}\left(B_{R} \backslash \overline{B_{a}}\right)$ the space of all those $u \in L^{q}\left(B_{R} \backslash \overline{B_{a}}\right)$ which are radially symmetric. We also write $L_{\mathrm{r}}^{q}(a, R)$ for this space when any $u \in L_{\mathrm{r}}^{q}\left(B_{R} \backslash \overline{B_{a}}\right)$ is regarded as a function on $(a, R)$. We define the norm on $L_{\mathrm{r}}^{q}(a, R)$ by

$$
\|u\|_{L_{\mathrm{r}}^{q}(a, R)}:=\left(\int_{a}^{R}|u(r)|^{q} r^{N-1} \mathrm{~d} r\right)^{1 / q} \quad \text { if } q<\infty
$$


and $\|u\|_{L_{\mathrm{r}}^{q}(a, R)}:=\|u\|_{L^{\infty}(a, R)}$ if $q=\infty$, which is obviously equivalent to the original norm on $L_{\mathrm{r}}^{q}\left(B_{R} \backslash \overline{B_{a}}\right)$. Moreover, we define the norm on $W_{\mathrm{r}}^{2, q}(a, R)$ by

$$
\|u\|_{W_{\mathrm{r}}^{2, q}(a, R)}:=\|u\|_{L_{\mathrm{r}}^{q}(a, R)}+\left\|u^{\prime} / r\right\|_{L_{\mathrm{r}}^{q}(a, R)}+\left\|u^{\prime \prime}\right\|_{L_{\mathrm{r}}^{q}(a, R)} \quad \text { if } q<\infty
$$

and $\|u\|_{W_{\mathrm{r}}^{2, q}(a, R)}:=\|u\|_{W^{2, \infty}(a, R)}$ if $q=\infty$, which is equivalent to the norm on $W_{\mathrm{r}}^{2, q}\left(B_{R} \backslash \overline{B_{a}}\right)$. See [25, Lemma 6.1] for this equivalence.

In this and next sections we often deal with functions $u \in W_{\mathrm{r}}^{2, q}(a, R)$, with $a \in$ $(0, R)$ and $q>N / 2$, satisfying $u^{\prime}(a)=0$ and, without further comment, we use the convention that such a function $u$ is identified with its extension $\tilde{u} \in W_{\mathrm{r}}^{2, q}(0, R)$ defined by $\tilde{u}(r)=u(a)$ for $r \in(0, a)$ and $\tilde{u}(r)=u(r)$ for $r \in[a, R]$.

We remark, thanks to (F2) and (F4), that for a.e. $r \in(0, R)$ and all $\left(\omega, m_{i}, p_{i}, u_{i}\right) \in$ $\mathbf{S}^{N-1} \times \mathbb{R}^{3}$ with $i=1,2$, we have

$$
\begin{aligned}
\mathcal{F} & \left(m_{1}, p_{1}, u_{1}, r\right)-\mathcal{F}\left(m_{2}, p_{2}, u_{2}, r\right) \\
& \leq P^{+}\left(\left(m_{1}-m_{2}\right) \omega \otimes \omega+r^{-1}\left(p_{1}-p_{2}\right)\left(I_{N}-\omega \otimes \omega\right)\right) \\
& +\beta(r \omega)\left|p_{1}-p_{2}\right|+\gamma(r \omega)\left|u_{1}-u_{2}\right| .
\end{aligned}
$$

Noting that the functions

$$
\omega \mapsto P^{ \pm}\left(m \omega \otimes \omega+r^{-1} p\left(I_{N}-\omega \otimes \omega\right)\right)
$$

are constant for all $(m, p, r)$, we set

$$
\mathcal{P}^{ \pm}(m, p, r)=P^{ \pm}\left(m \omega \otimes \omega+r^{-1} p\left(I_{N}-\omega \otimes \omega\right)\right)
$$

for $(m, p, r) \in \mathbb{R}^{2} \times(0, R)$, and integrating the inequality above over the unit sphere $\mathbf{S}^{N-1}$ with respect to the surface measure, for a.e. $r \in(0, R)$ and all $\left(m_{i}, p_{i}, u_{i}\right) \in \mathbb{R}^{3}$, with $i=1,2$, we obtain

$$
\begin{aligned}
& \mathcal{F}\left(m_{1}, p_{1}, u_{1}, r\right)-\mathcal{F}\left(m_{2}, p_{2}, u_{2}, r\right) \\
& \leq \mathcal{P}^{+}\left(m_{1}-m_{2}, p_{1}-p_{2}, r\right)+\bar{\beta}(r)\left|p_{1}-p_{2}\right|+\bar{\gamma}(r)\left|u_{1}-u_{2}\right| .
\end{aligned}
$$

Here $\bar{\beta}(r)$ and $\bar{\gamma}(r)$ denote the averages of $\beta(r \omega)$ and $\gamma(r \omega)$, respectively, over $\mathbf{S}^{N-1}$ with respect to the surface measure, that is,

$$
\bar{\beta}(r):=\alpha_{N}^{-1} \int_{\mathbf{S}^{N-1}} \beta(r \omega) \mathrm{d} S \quad \text { and } \quad \bar{\gamma}(r):=\alpha_{N}^{-1} \int_{\mathbf{S}^{N-1}} \gamma(r \omega) \mathrm{d} S,
$$

where $\mathrm{d} S$ and $\alpha_{N}$ denote the $(N-1)$-dimensional surface measure and the area of the sphere $\mathbf{S}^{N-1}$, respectively, and the functions $\bar{\beta}$ and $\bar{\gamma}$ belong to $L_{\mathrm{r}}^{q}(0, R)$. Indeed, the inequalities 


$$
\|\bar{\beta}\|_{L_{\mathrm{r}}^{q}(0, R)} \leq \alpha_{N}^{-1 / q}\|\beta\|_{L^{q}\left(B_{R}\right)} \quad \text { and } \quad\|\bar{\gamma}\|_{L_{\mathrm{r}}^{q}(0, R)} \leq \alpha_{N}^{-1 / q}\|\gamma\|_{L^{q}\left(B_{R}\right)}
$$

hold. For instance, the first inequality can be checked, with use of Hölder's inequality, as follows:

$$
\begin{aligned}
\|\bar{\beta}\|_{L_{\mathrm{r}}^{q}(0, R)}^{q} & =\int_{0}^{R}\left(\alpha_{N}^{-1} \int_{\mathbf{S}^{N-1}} \beta(r \omega) \mathrm{d} S\right)^{q} r^{N-1} \mathrm{~d} r \\
& \leq \alpha_{N}^{-1} \int_{0}^{R} \int_{\mathbf{S}^{N-1}} \beta(r \omega)^{q} \mathrm{~d} S r^{N-1} \mathrm{~d} r=\alpha_{N}^{-1}\|\beta\|_{L^{q}\left(B_{R}\right)}^{q} .
\end{aligned}
$$

For any $\omega \in \mathbf{S}^{N-1}$, we set $M:=I_{N}-\omega \otimes \omega$ and observe that $M \geq 0$ and $\operatorname{tr} M=N-1$, to deduce that $P^{+}(M) \leq(N-1) \Lambda$. Hence, we have

$$
\mathcal{P}^{+}(m, p, r) \leq P_{1}^{+}(m)+(N-1) \Lambda|p| / r \quad \text { for all }(m, p, r) \in \mathbb{R}^{2} \times(0, R),
$$

where $P_{1}^{+}$denotes the one-dimensional Pucci operator.

Here an important remark is that under the assumptions (F2) and (F4), the function $\mathcal{F}$ satisfies (F2) and (F4) on the interval $[a, R]$ for any $a \in(0, R)$. However, because of the factor $1 / r$ in the last term in $(62), \mathcal{F}$ does not satisfy $(\mathrm{F} 2)$ on $[0, R]$.

In what follows we write $\mathcal{F}[u]$ and $\mathcal{P}^{+}[u]$ for $\mathcal{F}\left(u^{\prime \prime}(r), u^{\prime}(r), u(r), r\right)$ and $\mathcal{P}^{+}\left(u^{\prime \prime}(r), u^{\prime}(r), r\right)$, respectively.

For later reference, we remark that if $(\mu, u) \in \mathbb{R} \times W_{\mathrm{r}}^{2, q}(a, R)$ is an eigenpair of (7) in $(a, R)$, then both $\varphi=u$ and $\varphi=-u$ satisfy

$$
\mathcal{P}^{+}[\varphi]+\bar{\beta}\left|\varphi^{\prime}\right|+\bar{\gamma}|\varphi|+\mu \varphi \geq 0 \quad \text { in }(a, R) .
$$

Now, we recall some facts from [25, Lemmas 7.1, 7.2, Theorems 7.5, 7.6 and 7.7].

Lemma 32 Assume that (F2) and (F5) hold. Let $a \in[0, R), u \in W_{\mathrm{r}}^{2, q}(a, R)$ and $f, f_{1}, f_{2} \in L_{\mathrm{r}}^{q}(a, R)$. Assume that $u^{\prime}(a)=0$ if $a>0$. (1) If $u$ satisfies $\mathcal{P}^{+}[u]+$ $\bar{\beta}\left|u^{\prime}\right|+f \geq 0$ in $(a, R)$, then there exists a constant $C>0$ depending only on $\lambda, \Lambda, q, N$ and $\|\bar{\beta}\|_{L_{\mathrm{r}}^{N}(0, R)}$ such that

$$
\left\|\left(\frac{u^{\prime}}{r}\right)_{-}\right\|_{L_{\mathrm{r}}^{q}(a, R)} \leq C\left\|f_{+}\right\|_{L_{\mathrm{r}}^{q}(a, R)} .
$$

Furthermore,

$$
\max _{t \in[a, R]} u(t)-u(R) \leq C\left(R^{(2 q-N) /(q-1)}-a^{(2 q-N) /(q-1)}\right)^{(q-1) / q}\left\|f_{+}\right\|_{L_{\mathrm{r}}^{q}(a, R)} .
$$

(2) If $u(R)=0$ and $u$ satisfies

$$
\mathcal{P}^{+}[u]+\bar{\beta}\left|u^{\prime}\right|+f_{1} \geq 0 \quad \text { and } \quad \mathcal{P}^{-}[u]-\bar{\beta}\left|u^{\prime}\right|-f_{2} \leq 0 \quad \text { in }(a, R),
$$


then there exists a constant $C>0$ depending only on $q, \lambda, \Lambda, N, R,\|\bar{\beta}\|_{L_{\mathrm{r}}^{N}(0, R)}$ and $\|\bar{\beta}\|_{L_{\mathrm{r}}^{q}(0, R)}$ such that

$$
\|u\|_{W_{\mathrm{r}}^{2, q}(a, R)} \leq C\left(\left\|\left(f_{1}\right)_{+}\right\|_{L_{\mathrm{r}}^{q}(a, R)}+\left\|\left(f_{2}\right)_{+}\right\|_{L_{\mathrm{r}}^{q}(a, R)}\right) .
$$

(3) Assume $a=0$ and that $u \geq 0$ in $[0, R]$ and $\mathcal{P}^{-}[u]-\bar{\beta}\left|u^{\prime}\right|-\bar{\gamma} u \leq 0$ in $(0, R)$. Then either $u \equiv 0$ in $[0, R]$ or $u>0$ in $[0, R)$ and $\max \left\{u(R),-u^{\prime}(R)\right\}>0$.

A few comments regarding the proof of the lemma above may be in order. The inequality (64) above is proved in the first half of the proof of Theorem 7.5 in [25], and the latter of assertion (1) follows form Lemma 7.2 in [25] applied to the function $u(r)-u(R)$. The inequality, $\max \left\{u(R),-u^{\prime}(R)\right\}>0$, in assertion (3) follows from Proposition 13, applied on an interval $[a, R]$, with $a \in(0, R)$.

Proposition 33 Assume $(\mathrm{F} 1)-(\mathrm{F} 5)$ hold. Let $(\mu, u) \in \mathbb{R} \times W_{\mathrm{r}}^{2, q}(0, R)$ be a solution of (7). If $u \neq \equiv$, then $u(0) \neq 0$.

Proof By Lemma 32 (1) and (63), if $u(b)=0$ for some $b \in(0, R]$, we obtain

$$
\max _{[0, b]}|u| \leq C b^{2-N / q}\|(\bar{\gamma}+|\mu|)|u|\|_{L_{\mathrm{r}}^{q}(0, b)} \leq C b^{2-N / q}\|\bar{\gamma}+|\mu|\|_{L_{\mathrm{r}}^{q}(0, b)} \max _{[0, b]}|u|,
$$

where $C$ is a positive constant depending only on $\lambda, \Lambda, q, N$ and $\|\bar{\beta}\|_{L_{\mathrm{r}}^{N}(0, R)}$. We thus get

$$
\left(1-C_{1} b^{2-N / q}\right) \max _{[0, b]}|u| \leq 0,
$$

where $C_{1}:=C\|\bar{\gamma}+|\mu|\|_{L_{\mathrm{r}}^{q}(0, b)}$. Note that $\max _{[0, b]}|u|>0$. Indeed, if we assume that $\max _{[0, b]}|u|=0$, then $u(b)=u^{\prime}(b)=0$. Hence, for each $c \in(0, R)$, applying Lemma 12 and invoking (27) on $[c, R]$, we see that $u(x) \equiv 0$ on $[c, R]$ which, however, is a contradiction. From (65) we get $C_{1} b^{2-N / q} \geq 1$. Thus, choosing $c \in(0, R)$ so that $C_{1} c^{2-N / q}<1$, we have either $u(x)>0$ for all $x \in(0, c)$ or else $u(x)<0$ for all $x \in(0, c)$. Lemma 32 (3) now ensures that $u(0) \neq 0$.

The following maximum principle and comparison principle are valid.

Theorem 34 Assume that (F1)-(F5) hold. (1) Let $\theta \in(0, \pi]$, and assume that there exists a function $\psi \in W_{\mathrm{r}}^{2, q}(0, R)$ such that

$$
\left\{\begin{array}{l}
\mathcal{F}[\psi] \leq 0, \quad \mathcal{F}[\psi] \not \equiv 0 \text { and } \psi>0 \quad \text { in }(0, R) \\
B^{+}(\psi, R) \in l(\theta)
\end{array}\right.
$$

If $u \in W_{\mathrm{r}}^{2, q}(0, R)$ satisfies

$$
\left\{\begin{array}{l}
\mathcal{F}[u] \geq 0 \quad \text { in }(0, R) \\
B^{+}(u, R) \in \overline{l(\tau)} \text { for some } \tau \in[\theta, 2 \pi]
\end{array}\right.
$$


then $u \leq 0$ in $(0, R)$. (2) Let $u, v \in W_{\mathrm{r}}^{2, q}(0, R), \theta_{u} \in(0,2 \pi]$ and $\theta_{v} \in(0, \pi]$. Assume that $\mathcal{F}[v] \leq \mathcal{F}[u]$ in $(0, R), B^{+}(u, R) \in \overline{l\left(\theta_{u}\right)}$ and $B^{+}(v, R) \in l\left(\theta_{v}\right)$ and that $v>0$ in $(0, R), \sup _{(0, R)} u / v=1$ and $\theta_{u} \geq \theta_{v}$. Then $u \equiv v$ in $[0, R]$. (3) Let $\theta \in(0, \pi]$ and $v, w \in W_{\mathrm{r}}^{2, q}(0, R)$. Assume that there exists $\psi \in W_{\mathrm{r}}^{2, q}(0, R)$ satisfying (66) and that $v, w$ satisfy

$$
\left\{\begin{array}{l}
\mathcal{F}[w] \leq 0, \quad \mathcal{F}[w] \leq \mathcal{F}[v] \text { and } w>0 \text { in }(0, R), \\
B^{+}(w, R) \in l(\theta), \quad B^{+}(v, R) \in \overline{l(\tau)} \text { for some } \tau \in[\theta, 2 \pi] .
\end{array}\right.
$$

Then $v \leq w$ in $[0, R]$.

An important consequence of claim (1) of the theorem above is that if $\mu<\mu_{\mathrm{r}}^{0}(\theta, R)$ and $u \in W_{\mathrm{r}}^{2, q}(0, R)$ satisfies $\mathcal{F}[u]+\mu u \geq 0$ in $(0, R)$ and $B^{+}(u, R) \in \overline{l(\tau)}$ for some $\tau \in[\theta, 2 \pi]$, then $u \leq 0$ in $[0, R]$. Indeed, choosing $\psi \in W_{\mathrm{r}}^{2, q}(0, R)$ to be an eigenfunction corresponding to $\mu_{\mathrm{r}}^{0}(\theta, R)$, we have $\mathcal{F}[\psi]+\mu \psi<0$ and $\psi>0$ in $(0, R)$.

Proof We first show assertion (2). To show (2), we suppose to the contrary that $u \neq \equiv v$ on $[0, R]$, and note by assumption that $u \leq v$ in $[0, R]$. Setting $w:=v-u \geq 0$, we get $\mathcal{P}^{-}[w]-\bar{\beta}\left|w^{\prime}\right|-\bar{\gamma} w \leq 0$ in $(0, R)$. Since $w \not \equiv 0$ in $[0, R]$, Lemma 32 (3) assures that $w>0$ in $[0, R)$ and $\max \left\{w(R),-w^{\prime}(R)\right\}>0$. When $w(R)>0$, it is easily seen that $\sup _{(0, R)} u / v<1$, which is a contradiction.

When $w(R)=0$, we have two cases: either $v(R)=u(R)>0$, or $v(R)=u(R)=$ 0 . If $v(R)=u(R)>0$, then we have $u^{\prime}(R)>v^{\prime}(R)$, which yields the inequality $\theta_{u}<\theta_{v}$, a contradiction. If $v(R)=u(R)=0$, then we get $\theta_{v}=\pi, v^{\prime}(R)<0$ and $v^{\prime}(R)<u^{\prime}(R)$. Hence, by l'Hôpital's rule, we find that

$$
\lim _{r \rightarrow R+0} \frac{u(r)}{v(r)}=\frac{u^{\prime}(R)}{v^{\prime}(R)}<1,
$$

which gives a contradiction, $\sup _{(0, R)} u / v<1$. Thus, $w \equiv 0$ in $[0, R]$ and assertion (2) holds.

Next, we prove assertion (1). To show (1), we note first that $\mathcal{P}^{-}[\psi]-\bar{\beta}\left|\psi^{\prime}\right|-\bar{\gamma} \psi \leq$ $\mathcal{F}[\psi] \leq 0$ in $(0, R)$ and by Lemma $32(3)$ that $\psi(0)>0$. Suppose to the contrary that $\max _{[0, R]} u>0$, set $\rho=\sup _{[0, R)} u / \psi$ and observe that $\rho \in(0, \infty)$. Noting $\mathcal{F}[\rho \psi]=\rho \mathcal{F}[\psi] \leq 0 \leq \mathcal{F}[u]$, assertion (2) gives $\rho \psi \equiv u$ in $[0, R]$. Therefore, we infer $\mathcal{F}[\psi] \equiv 0$, however, this is a contradiction. Thus assertion (1) is valid.

In order to prove (3), we argue by contradiction and suppose $\max _{[0, R]}(v-$ $w)>0$. As in the proof of assertion (1), we obtain $\psi(0)>0, w(0)>0$ and $\max \left\{w(R),-w^{\prime}(R)\right\}>0$. Observe as in the proof of Proposition 18 that if $w(R)=0$, then $w^{\prime}(R)<0$ and $v(R) \leq 0$. We set $\rho:=\sup _{(0, R)} v / w$ and argue as in the proof of Proposition 18, with Lemma 32 (3) as the strong maximum principle, to find that $\rho \in(1, \infty), \rho w \equiv v$ on $[0, R]$ and $F[w]=0$ in $(0, R)$. Moreover, using assertion (1) above, we obtain $w \leq 0$, a contradiction. The proof is complete.

Let $0<a<R$ and consider the eigenvalue problem for (7) in $(a, R)$ with boundary condition $u^{\prime}(a)=0$ and $B^{+}(u, R) \in l\left(\theta^{+}\right)$. The Neumann condition 
$u^{\prime}(a)=0$ may be stated in terms of a unilateral boundary condition as $B(u, a, R) \in$ $L\left(\theta^{-}, \theta^{+}\right)$, where $\theta^{-} \in\{\pi / 2,3 \pi / 2\}$.

In what follows we use the notation $\mu_{\mathrm{r}}^{n}(\theta, a, R)$ to denote the $n$th order eigenvalue of (7) with the boundary condition,

$$
B(u, a, R) \in L(\theta) .
$$

Lemma 35 Assume that (F1)-(F5) hold. Let $c \in(0, R)$ and $(n, \theta)=\left(n, \theta^{-}, \theta^{+}\right) \in$ $\mathbb{N}_{0} \times\{\pi / 2,3 \pi / 2\} \times(0,2 \pi]$. If $(n, \theta)$ is admissible, then $\sup _{a \in(0, c)}\left|\mu_{\mathrm{r}}^{n}(\theta, a, R)\right|<$ $\infty$.

Proof In view of (S1), it is enough to treat the case where $\theta^{+} \in(0, \pi]$. For each $a \in(0, c)$ let $\varphi_{a} \in W^{2,1}(a, R)$ be an $n$th order eigenfunction of (7) and (67).

Note that $(n+2, \theta)$ is admissible and set $v_{0}=\mu_{\mathrm{r}}^{n+2}(\theta, c, R)$. We fix any $a \in(0, c)$ and show that $\mu_{\mathrm{r}}^{n}(\theta, a, R) \leq \nu_{0}$. Select $\theta_{a, c}^{-} \in(0,2 \pi]$ so that $B^{-}\left(\varphi_{a}, c\right) \in l\left(\theta_{a, c}^{-}\right)$, that is, $\theta_{a, c}^{-}=\Theta\left(-\varphi_{a}^{\prime}(c), \varphi_{a}(c)\right)$, and set $\theta_{a, c}=\left(\theta_{a, c}^{-}, \theta^{+}\right)$, and observe that the pair of $\mu_{\mathrm{r}}^{n}(\theta, a, R)$ and $\varphi_{a}$ is an eigenpair of (7) and (67), with the interval $(a, R)$ and the angles $\theta$ replaced by $(c, R)$ and $\theta_{a, c}$, respectively. Let $n_{a, c} \in \mathbb{N}_{0}$ be the number of zeroes of $\varphi_{a}$ in $(c, R)$, and note that $n_{a, c} \leq n$ and $\mu_{\mathrm{r}}^{n_{a, c}}\left(\theta_{a, c}, c, R\right)=\mu_{\mathrm{r}}^{n}(\theta, a, R)$. We also remark that $\left(n_{a, c}, \theta_{a, c}\right) \leq(n+2, \theta)$ holds from the remark stated after (11)-(15). Thus, by Theorem 4 , we get $\mu_{\mathrm{r}}^{n_{a, c}}\left(\theta_{a, c}, c, R\right) \leq v_{0}$ and hence $\mu_{\mathrm{r}}^{n}(\theta, a, R) \leq v_{0}$.

Next we give a lower bound of $\mu_{\mathrm{r}}^{n}(\theta, a, R)$, with $a \in(0, c)$. Set $\theta_{0}=\left(\pi / 2, \theta^{+}\right)$, and note that $\left(0, \theta_{0}\right)$ is admissible and by Theorem 4 that $\mu_{\mathrm{r}}^{0}\left(\theta_{0}, a, R\right) \leq \mu_{\mathrm{r}}^{n}(\theta, a, R)$. We need to find a lower bound, independent of $a$, of the $\mu_{\mathrm{r}}^{0}\left(\theta_{0}, a, R\right)$, with $a \in(0, c)$.

For each $a \in(0, c)$ let $\psi_{a} \in W^{2,1}(a, R)$ be a principal eigenfunction of (7) and (67), with the angles $\theta$ replaced by $\theta_{0}$. Clearly, $\psi_{a}>0$ in $(a, R)$ and the eigenvalue corresponding to $\psi_{a}$ is $\mu_{\mathrm{r}}^{0}\left(\theta_{0}, a, R\right)$. For each $r \in(a, R)$ we choose $\tau(r) \in(0, \pi)$ so that $B^{-}\left(\psi_{a}, r\right) \in l(\tau(r))$. That is, we fix $\tau(r) \in(0, \pi)$ by setting $\tau(r)=\Theta\left(-\psi_{a}^{\prime}(r), \psi_{a}(r)\right)$. Note that the pair of $\mu_{\mathrm{r}}^{0}\left(\theta_{0}, a, R\right)$ and $\psi_{a}$ is an eigenpair of (7) and (67), with the interval $(a, R)$ and angles $\theta$ replaced by $(r, R)$ and $\left(\tau(r), \theta^{+}\right)$, respectively, for all $r \in(a, R)$.

Let $\chi \in W^{2,1}(c, R)$ be a principal eigenfunction of (7) and (67), with the interval $(a, R)$ and angles $\theta=\left(\theta^{-}, \theta^{+}\right)$replaced by $(c, R)$ and $\left(\pi / 4, \theta^{+}\right)$, respectively. Note that $\chi>0$ on $[c, R)$. Set $v_{1}=\mu_{\mathrm{r}}^{0}\left(\pi / 4, \theta^{+}, c, R\right)$ and $\sigma(r)=\Theta\left(-\chi^{\prime}(r), \chi(r)\right)$ for $r \in[c, R)$, note that $B^{-}(\chi, r) \in l(\sigma(r))$ for all $r \in[c, R)$ and $\left(v_{1}, \chi\right)$ is a principal eigenpair of (7) and (67), with the interval $(a, R)$ and angles $\theta$ replaced by $(r, R)$ and $\left(\sigma(r), \theta^{+}\right)$, respectively, for any $r \in[c, R)$.

Since $r \mapsto \sigma(r)$ is continuous on $[c, R)$, we may select $d \in(c, R)$ so that $\sigma(r) \leq$ $\pi / 3$ for all $r \in[c, d]$. Noting that

$$
\mathcal{P}^{+}\left[\psi_{a}\right]+\bar{\beta}\left|\psi_{a}^{\prime}\right|+\left(\bar{\gamma}+\mu_{a}\right)_{+} \psi_{a} \geq 0 \quad \text { in }(a, R),
$$

we find by Lemma 32 (1) that there exists a constant $C>0$, depending only on $\lambda, \Lambda, q, N, c, d$ and $\|\bar{\beta}\|_{L_{\mathrm{r}}^{q}(0, d)}$ such that 


$$
\begin{aligned}
& \left\|\left(\frac{\psi_{a}^{\prime}}{r}\right)_{-}\right\|_{L_{\mathrm{r}}^{q}(c, d)} \leq C\left\|\left(\bar{\gamma}+\mu_{a}\right)_{+}\right\|_{L_{\mathrm{r}}^{q}(c, d)} \max _{[c, d]} \psi_{a}, \\
& \max _{[c, d]} \psi_{a} \leq \psi_{a}(d)+C\left\|\left(\bar{\gamma}+\mu_{a}\right)_{+}\right\|_{L_{\mathrm{r}}^{q}(c, d)} \max _{[c, d]} \psi_{a} .
\end{aligned}
$$

We set

$$
\delta(m)=C\left\|(\bar{\gamma}+m)_{+}\right\|_{L_{\mathrm{r}}^{q}(c, d)} \text { for } m \in \mathbb{R},
$$

and observe that $m \mapsto \delta(m)$ is nondecreasing on $\mathbb{R}$ and $\lim _{m \rightarrow-\infty} \delta(m)=0$. We choose $m_{0} \in \mathbb{R}$ so that

$$
\delta\left(m_{0}\right)<1 \quad \text { and } \quad \cos ^{-1}\left(\frac{\delta\left(m_{0}\right)}{\left(1-\delta\left(m_{0}\right)\right)\|1 / r\|_{L_{\mathrm{r}}^{q}(c, d)}}\right)>\pi / 3 .
$$

Recall that the function $\cos ^{-1}:[-1,1] \rightarrow[0, \pi]$ is nonincreasing and continuous and $\cos ^{-1}(0)=\pi / 2$.

We write $\mu_{a}=\mu_{\mathrm{r}}^{0}\left(\theta_{0}, a, R\right)$, for notational simplicity, and now show that $\mu_{a} \geq$ $\min \left\{v_{1}, m_{0}\right\}$ for all $a \in(0, c]$. To do this, we fix any $a \in(0, c]$. If $\mu_{a} \geq m_{0}$, there is nothing to show, and hence, in what follows, we assume $\mu_{a}<m_{0}$ and prove $v_{1} \leq \mu_{a}$.

From (68), (69) and the monotonicity of $\delta$, we get

$$
\min _{[c, d]}\left(\psi_{a}^{\prime}\right)_{-} \leq \delta\left(m_{0}\right)\|1 / r\|_{L_{\mathrm{r}}^{q}(c, d)}^{-1} \max _{[c, d]} \psi_{a} \quad \text { and } \quad\left(1-\delta\left(m_{0}\right)\right) \max _{[c, d]} \psi_{a} \leq \psi_{a}(d) .
$$

If $\min _{[c, d]} \psi_{a}<\left(1-\delta\left(m_{0}\right)\right) \max _{[c, d]} \psi_{a}$, then there exists $r_{a} \in[c, d)$ such that

$$
\psi_{a}\left(r_{a}\right)<\left(1-\delta\left(m_{0}\right)\right) \max _{[c, d]} \psi_{a} \leq \psi_{a}(d)
$$

which implies in view of the mean value theorem that $\psi_{a}^{\prime}\left(s_{a}\right)>0$ for some $s_{a} \in\left(r_{a}, d\right)$. Note that $\tau\left(s_{a}\right)>\pi / 2>\pi / 3 \geq \sigma\left(s_{a}\right)$. Theorem 4 assures that $\mu_{\mathrm{r}}^{0}\left(\tau\left(s_{a}\right), \theta^{+}, s_{a}, R\right) \geq \mu_{\mathrm{r}}^{0}\left(\sigma\left(s_{a}\right), \theta^{+}, s_{a}, R\right)$. That is, we have $\mu_{a} \geq v_{1}$.

Otherwise, we have $\min _{[c, d]} \psi_{a} \geq\left(1-\delta\left(m_{0}\right)\right) \max _{[c, d]} \psi_{a}$. Combining this with the first inequality of (70) yields

$$
\left(\psi_{a}^{\prime}\right)_{-}\left(t_{a}\right) \leq \frac{\delta\left(m_{0}\right)}{1-\delta\left(m_{0}\right)}\|1 / r\|_{L_{\mathrm{r}}^{q}(c, d)}^{-1} \psi_{a}\left(t_{a}\right)
$$

for some $t_{a} \in[c, d]$. Hence,

$$
\begin{aligned}
\tau\left(t_{a}\right)=\Theta\left(-\psi_{a}^{\prime}\left(t_{a}\right), \psi_{a}\left(t_{a}\right)\right) & \geq \cos ^{-1}\left(\frac{\frac{\delta\left(m_{0}\right)}{1-\delta\left(m_{0}\right)}\|1 / r\|_{L_{\mathrm{r}}^{q}(c, d)}^{-1} \psi_{a}\left(t_{a}\right)}{\sqrt{\psi_{a}^{\prime}\left(t_{a}\right)^{2}+\psi_{a}\left(t_{a}\right)^{2}}}\right) \\
& \geq \cos ^{-1}\left(\frac{\delta\left(m_{0}\right)}{1-\delta\left(m_{0}\right)}\|1 / r\|_{L_{\mathrm{r}}^{q}(c, d)}^{-1}\right)>\pi / 3 \geq \sigma\left(t_{a}\right) .
\end{aligned}
$$


Therefore, by Theorem 4, we find that $v_{1}=\mu_{\mathrm{r}}^{0}\left(\sigma\left(t_{a}\right), \theta^{+}, t_{a}, R\right) \leq \mu_{\mathrm{r}}^{0}\left(\tau\left(t_{a}\right), \theta^{+}, t_{a}, R\right)$ $=\mu_{a}$. Thus, we have $\mu_{a} \geq \min \left\{m_{0}, v_{1}\right\}$ for all $a \in(0, c]$. Furthermore, recalling that $\mu_{\mathrm{r}}^{0}(\theta, a, R) \leq \mu_{\mathrm{r}}^{n}(\theta, a, R) \leq \nu_{0}$, we conclude that $\sup _{a \in(0, c)}\left|\mu_{\mathrm{r}}^{n}(\theta, a, R)\right|$ $<\infty$.

We consider the boundary value problem

$$
\begin{aligned}
& \mathcal{F}[u]+\mu u+\operatorname{sgn}(u) f=0 \quad \text { in }(a, R), \\
& u^{\prime}(a)=0 \text { if } a>0, \quad \text { and } \quad B^{+}(u, R) \in l(\theta)
\end{aligned}
$$

where $\mu \in \mathbb{R}, f \in L_{\mathrm{r}}^{q}(0, R)$, and $\theta \in(0,2 \pi]$. The Neumann boundary condition at $a \in(0, R)$ is rephrased as $B^{-}(u, a) \in l(\pi / 2) \cup l(3 \pi / 2)$.

Lemma 36 Assume (F1)-(F5) hold. Let $\left\{\mu_{j}\right\}_{j \in \mathbb{N}} \subset \mathbb{R}, \mu \in \mathbb{R},\left\{a_{j}\right\} \subset(0, R), \theta \in$ $(0,2 \pi], n \in \mathbb{N}_{0},\left\{u_{j}\right\}_{j \in \mathbb{N}} \subset W_{\mathrm{r}}^{2, q}(0, R), u \in W_{\mathrm{r}}^{2, q}(0, R)$, and $f \in L_{\mathrm{r}}^{q}(0, R)$. Assume that, for every $j \in \mathbb{N}, u_{j}$ is an nth order solution of (71), with a and $\mu$ replaced by $a_{j}$ and $\mu_{j}$, respectively, that, as $j \rightarrow \infty, u_{j} \rightarrow u$ weakly (weakly star when $q=\infty)$ in $W_{\mathrm{r}}^{2, q}(0, R), \mu_{j} \rightarrow \mu$ and $a_{j} \rightarrow 0$, and that $u \neq \equiv 0$ on $[0, R]$. Then $u$ is an $n$th order solution of (71), with $a=0$.

Proof Due to the Sobolev embedding theorem, the sequence $\left\{u_{j}\right\}$ converges to $u$ in $C([0, R])$ as well as in $C_{\text {loc }}^{1}((0, R])$.

For $j \in \mathbb{N}$, let $\left\{r_{j, i}\right\}_{i=0}^{n+1} \subset(0, R]$ be the increasing sequence such that $r_{j, 0}=$ $a_{j}, r_{j, n+1}=R$ and $u_{j}\left(r_{j, i}\right)=0$ for all $i \in\{1, \ldots, n\}$. We may choose an increasing sequence $\left\{j_{k}\right\}_{k \in \mathbb{N}}$ of natural numbers so that as $k \rightarrow \infty$, the sequence $\left\{\left(r_{j_{k}, 0}, r_{j_{k}, 1}, \ldots, r_{j_{k}, n+1}\right)\right\}$ converges to a point $\left(r_{0}, r_{1}, \ldots, r_{n+1}\right) \in \mathbb{R}^{n+2}$. It is clear that $r_{0}=0 \leq r_{1} \leq \cdots \leq r_{n+1}=R$ and $u\left(r_{i}\right)=0$ for all $i \in\{1, \ldots, n\}$.

Observe that for any $i \in\{1, \ldots, n+1\}$, if $r_{i-1}<r_{i}$, then either

$$
\begin{aligned}
& \lim _{k \rightarrow \infty} \operatorname{sgn}\left(u_{j_{k}}(r)\right)=1 \text { for all } r \in\left(r_{i-1}, r_{i}\right), \text { or } \\
& \lim _{k \rightarrow \infty} \operatorname{sgn}\left(u_{j_{k}}(r)\right)=-1 \text { for all } r \in\left(r_{i-1}, r_{i}\right) .
\end{aligned}
$$

Hence, according to the Lebesgue convergence theorem, the sequence $\left\{\operatorname{sgn}\left(u_{j_{k}}\right) f\right\}$ converges, as $k \rightarrow \infty$, to a function $g$ in $L_{\mathrm{r}}^{q}(0, R)$ such that $|g|=f$ in $(0, R)$. Furthermore, for any $i \in\{2, \ldots, n+1\}$, if $0<r_{i-1}<r_{i}$, then we have either

$$
\begin{aligned}
& u \geq 0 \text { and } g=f \text { on }\left[r_{i-1}, r_{i}\right], \text { or } \\
& u \leq 0 \text { and } g=-f \text { on }\left[r_{i-1}, r_{i}\right] .
\end{aligned}
$$

By Lemma 24, for each $a \in(0, R)$, we have $\mathcal{F}[u]+\mu u+g=0$ in $(a, R)$, which readily yields

$$
\mathcal{F}[u]+\mu u+g=0 \quad \text { in }(0, R) .
$$

Because of the convergence of $\left\{u_{j}\right\}$ to $u$ in $C_{\text {loc }}^{1}((0, R])$, we deduce that, for $i \in$ $\{2, \ldots, n+1\}$

$$
\text { if } 0<r_{i-1}=r_{i} \text {, then }\left(u\left(r_{i}\right), u^{\prime}\left(r_{i}\right)\right)=(0,0) \text {. }
$$


Indeed, for $i \in\{2, \ldots, n\}$, by the mean value theorem, we have $0=\left(u_{j}\left(r_{j, i}\right)-\right.$ $\left.u_{j}\left(r_{j, i-1}\right)\right) /\left(r_{j, i}-r_{j, i-1}\right)=u_{j}^{\prime}\left(z_{j, i}\right)$ for some $z_{j, i} \in\left(r_{j, i-1}, r_{j, i}\right)$, and, by putting $j=j_{k}$ and sending $k \rightarrow \infty$, we obtain $u^{\prime}\left(r_{i}\right)=0$. Similarly, by the boundary condition at $r=R$, if $r_{i}=R$ for $i \leq n$, then we have $\left(u\left(r_{i}\right), u^{\prime}\left(r_{i}\right)\right)=(0,0)$.

By the strong maximum principle, we infer that for $i \in\{2, \ldots, n+1\}$,

$$
\begin{aligned}
& \text { if } 0<r_{i-1}<r_{i} \text { and } u \neq 0 \text { in }\left(r_{i-1}, r_{i}\right), \text { then }|u|>0 \text { in }\left(r_{i-1}, r_{i}\right), \\
& \left(u\left(r_{i-1}\right), u^{\prime}\left(r_{i-1}\right)\right) \neq(0,0) \text { and }\left(u\left(r_{i}\right), u^{\prime}\left(r_{i}\right)\right) \neq(0,0) .
\end{aligned}
$$

As noted before, the condition, $|u|>0$ in $\left(r_{i-1}, r_{i}\right)$, is equivalent to stating that either $u>0$ in $\left(r_{i-1}, r_{i}\right)$ or $u<0$ in $\left(r_{i-1}, r_{i}\right)$.

To examine that (75) holds, we may assume in view of (S1) and (72) that $u \geq 0$ and $g=f$ in $\left(r_{i-1}, r_{i}\right)$, where $0<r_{i-1}<r_{i}$. Noting by (73) that $\mathcal{F}[u]+\mu u \leq 0$ in $\left(r_{i-1}, r_{i}\right)$ and applying Proposition 13 to the functions 0 and $u$, we obtain $u>0$ in $\left(r_{i-1}, r_{i}\right), u^{\prime}\left(r_{i-1}\right)>0$ and $u^{\prime}\left(r_{i}\right)<0$, from which we conclude that (75) holds.

Similarly, by using Lemma 32 (3), the strong maximum principle in the radial case and the fact $u\left(r_{j}\right)=0$ for $1 \leq j \leq n$, we deduce that for $i \in\{1, \ldots, n+1\}$,

$$
\begin{gathered}
\text { if } 0=r_{i-1}<r_{i} \text { and } u \quad \equiv \quad 0 \text { in }\left(r_{i-1}, r_{i}\right) \text {, then } i=1,|u|>0 \text { in }\left[0, r_{i}\right) \\
\text { and }\left(u\left(r_{i}\right), u^{\prime}\left(r_{i}\right)\right) \neq(0,0) .
\end{gathered}
$$

We note that

$$
\text { if }\left(u(R), u^{\prime}(R)\right) \neq(0,0) \text {, then } B^{+}(u, R) \in l(\theta) \text {. }
$$

This is an immediate consequence of the fact that $\left(u(R), u^{\prime}(R)\right)=\lim _{j \rightarrow \infty}\left(u_{j}\right.$ $\left.(R), u_{j}^{\prime}(R)\right) \in \overline{l(\theta)}$.

Now, we intend to prove that for any $i \in\{1, \ldots, n+1\}$, either of the following two conditions holds:

$$
\begin{aligned}
i= & 1, \quad r_{i-1}=0<r_{i}, \quad|u|>0 \text { in }\left[r_{i-1}, r_{i}\right) \text { and } \\
& \left(u\left(r_{i}\right), u^{\prime}\left(r_{i}\right)\right) \neq(0,0),
\end{aligned}
$$

or

$$
\begin{gathered}
i>1, \quad 0<r_{i-1}<r_{i}, \quad|u|>0 \text { in }\left(r_{i-1}, r_{i}\right) \\
\left(u\left(r_{i-1}\right), u^{\prime}\left(r_{i-1}\right)\right) \neq(0,0) \quad \text { and } \quad\left(u\left(r_{i}\right), u^{\prime}\left(r_{i}\right)\right) \neq(0,0) .
\end{gathered}
$$

To see this, we set

$$
I=\{i \in\{1, \ldots, n+1\} \text { : either (78) or (79) holds }\}
$$

and show first that $I \neq \emptyset$.

By assumption, we have $u \neq \equiv$ in $(0, R)$. Hence, there exists an $i \in\{1, \ldots, n+1\}$ such that $r_{i-1}<r_{i}$ and $u \neq \equiv 0$ in $\left(r_{i-1}, r_{i}\right)$. It follows from (75) and (76) that $i \in I$. Thus, $I \neq \emptyset$. 
Next, to show that $I=\{1, \ldots, n+1\}$, we suppose to the contrary that $I \neq$ $\{1, \ldots, n+1\}$. We may then find $i \in I$ and $j \in\{1, \ldots, n+1\} \backslash I$ such that $|i-j|=1$. Consider the case when $j=i-1$. Since $i \in I$ and $i=j+1>1$, we have $r_{j}=r_{i-1}>0$ and $\left(u\left(r_{j}\right), u^{\prime}\left(r_{j}\right)\right) \neq(0,0)$. Hence, we have $r_{j-1}<r_{j}$ by (74) and $u \not \equiv 0$ on $\left[r_{j-1}, r_{j}\right]$. It follows now from (75) and (76) that $j \in I$, which is a contradiction.

We consider next the case when $j=i+1$. Since $i \in I$, we have $\left(u\left(r_{i}\right), u^{\prime}\left(r_{i}\right)\right) \neq$ $(0,0)$, which implies together with (74) that $r_{i}=r_{j-1}<r_{j}$. It follows from (75) that $j \in I$, which is a contradiction.

We have thus proved that $I=\{1, \ldots, n+1\}$. It is now clear that $u$ has exactly $n$ zeroes in $(0, R)$ and $u(0)>0$. This property of $u$ and (72) ensure that $g=\operatorname{sgn}(u) f$ in $(0, R)$. Moreover, we see from (77) that $B^{+}(u, R) \in l(\theta)$. Thus, $u$ is an $n$th order solution of (71), with $a=0$.

\section{Proofs of the main results in the radial case}

Proof of Theorem 9 For $i=1,2$, let $\left(\mu_{i}, \varphi_{i}\right) \in \mathbb{R} \times W_{\mathrm{r}}^{2, q}(0, R)$ be an $n$th order eigenpairs of (7)-(8), with $\theta$ replaced by $\theta_{i}$. Note by Proposition 33 that $\varphi_{i}(0) \neq 0$ for $i=1,2$.

We argue by contradiction and thus suppose that $\mu_{1}>\mu_{2}$.

Let $\left\{x_{j}\right\}_{j=0}^{n_{1}+1},\left\{y_{j}\right\}_{j=0}^{n_{2}+1}$ be the increasing sequences of points in $[0, R]$ such that $x_{0}=y_{0}=0, x_{n_{1}+1}=y_{n_{2}+1}=R$, and the $x_{j}$, with $0<j<n_{1}+1$, and the $y_{j}$, with $0<j<n_{2}+1$, are zeroes of the functions $\varphi_{1}$ and $\varphi_{2}$, respectively.

We assume first that $n_{1}=n_{2}, i\left(\theta_{1}\right)=i\left(\theta_{2}\right)$ and $\theta_{1} \leq \theta_{2}$. We note that $\varphi_{1}(0) \varphi_{2}(0)>0$, that $x_{n_{1}+1}=y_{n_{1}+1}=R$, set $m=\min \left\{j \in\left\{1, \ldots, n_{1}+1\right\}\right.$ : $\left.y_{j} \leq x_{j}\right\}$ and observe that $x_{m-1} \leq y_{m-1}<y_{m} \leq x_{m}$ and $\varphi_{1}(r) \varphi_{2}(r)>0$ for all $r \in\left(y_{m-1}, y_{m}\right)$.

We consider next the case when $n_{2}=n_{1}+1, i\left(\theta_{1}\right)=i\left(\theta_{2}\right)$ and $\theta_{1} \leq \theta_{2}$. We note that $\varphi_{1}(0) \varphi_{2}(0)<0$ and $y_{n_{1}+2}=R=x_{n_{1}+1}$, set $m=\min \left\{j \in\left\{2, \ldots, n_{1}+2\right\}\right.$ : $\left.y_{j} \leq x_{j-1}\right\}$ and observe that $x_{m-2}<y_{m-1}<y_{m} \leq x_{m-1}$ and $\varphi_{1} \varphi_{2}>0$ in $\left(y_{m-1}, y_{m}\right)$.

Consider now the case when $n_{2}=n_{1}+1$ and $i\left(\theta_{1}\right) \neq i\left(\theta_{2}\right)$. Note that $\varphi_{1}(0) \varphi_{2}(0)>$ 0 , set $m=\min \left\{j \in\left\{1, \ldots, n_{1}+1\right\}: y_{j} \leq x_{j}\right\}$ and observe that $x_{m-1} \leq y_{m-1}<$ $y_{m} \leq x_{m}, y_{m}<y_{n_{2}+1}=R$ and $\varphi_{1} \varphi_{2}>0$ in $\left(y_{m-1}, y_{m}\right)$.

What remains is the case where $n_{2} \geq n_{1}+2$. If $\varphi_{1}(0) \varphi_{2}(0)>0$, then set $m=$ $\min \left\{j \in\left\{1, \ldots, n_{1}+1\right\}: y_{j} \leq x_{j}\right\}$ and observe that $x_{m-1} \leq y_{m-1}<y_{m} \leq$ $x_{m}, y_{m}<R$ and $\varphi_{1} \varphi_{2}>0$ in $\left(y_{m-1}, y_{m}\right)$. If $\varphi_{1}(0) \varphi_{2}(0)<0$, then set $m=\min \{j \in$ $\left.\left\{2, \ldots, n_{1}+2\right\}: y_{j} \leq x_{j-1}\right\}$ and observe that $x_{m-2} \leq y_{m-1}<y_{m} \leq x_{m-1}, y_{m}<R$ and $\varphi_{1} \varphi_{2}>0$ in $\left(y_{m-1}, y_{m}\right)$.

Thus there exists a nonempty subinterval $[c, d] \subset[0, R]$ having the properties: (1) $\varphi_{1} \varphi_{2}>0$ in $(c, d)$, (2) if $c \neq 0$, then $\varphi_{2}(c)=0$ (3) if $d \neq R$, then $\varphi_{2}(R)=0$ and (4) if $d=R$, then $i\left(\theta_{1}\right)=i\left(\theta_{2}\right)$ and $\theta_{1} \leq \theta_{2}$.

By the symmetry (S1) we may assume that $\varphi_{1}>0$ and $\varphi_{2}>0$ in $(c, d)$. Since $\mu_{2}<\mu_{1}$, we get $\mathcal{F}\left[\varphi_{1}\right]+\mu_{2} \varphi_{1}<0$ in $(c, d)$. 
We use the maximum principle, Theorem 34 (1) if $c=0$ and Theorem 16 if $c>0$, to obtain $\varphi_{2} \leq 0$ on $[c, d]$, which is a contradiction. Thus, we see that the inequality, $\mu_{1} \leq \mu_{2}$, holds.

Comment on the proof of Theorem 7 (1) We do not give the proof of claim (1) of Theorem 7 since it is similar to that of Theorem 2 (1). Indeed, the uniqueness of $n$th order (radial) eigenvalue of (7) and (8) is a consequence of Theorem 9. Regarding uniqueness of $n$th order (normalized and radial) eigenfunctions, using the strong maximum principle, Theorem 32 (2) and Lemma 14, one may easily adapt the proof of Theorem 2 (1). We leave it to the reader to check the details.

Comment on the proof of Theorem 10 We do not give the proof of Theorem 10 since it is similar to that of Theorem 5, and we leave it to the interested reader to check the details.

Proof of Theorem 7 (2) Let $(n, \theta) \in \mathbb{N}_{0} \times(0,2 \pi]$. We select $\theta^{-} \in\{\pi / 2,3 \pi / 2\}$ so that $\left(n, \theta^{-}, \theta\right)$ is admissible, and fix a sequence $\left\{a_{j}\right\}_{j \in \mathbb{N}} \subset(0, R / 2)$ so that $\lim _{j \rightarrow \infty} a_{j}=0$. For each $j \in \mathbb{N}$, set $\mu_{j}=\mu_{\mathrm{r}}^{n}\left(\theta^{-}, \theta, a_{j}, R\right)$ and let $\varphi_{j} \in W_{\mathrm{r}}^{2, q}(0, R)$ be the eigenfunction corresponding to $\mu_{\mathrm{r}}^{n}\left(\theta^{-}, \theta, a_{j}, R\right)$, with $\left\|\varphi_{j}\right\|_{L^{\infty}(0, R)}=1$.

Thanks to Lemma 35, we see that the sequence $\left\{\mu_{j}\right\}_{j \in \mathbb{N}}$ is bounded. By taking a subsequence of $\left\{a_{j}\right\}$ if needed, we may assume that $\left\{\mu_{j}\right\}_{j \in \mathbb{N}}$ is convergent, and we set $\mu:=\lim _{j \rightarrow \infty} \mu_{j}$.

Noting that $\pm\left(\varphi_{j}(r)-\varphi_{j}(R)\right)$ satisfy

$$
\mathcal{P}^{+}[u]+\bar{\beta}(r)\left|u^{\prime}\right|+f_{j}(r) \geq 0 \quad \text { in }\left(a_{j}, R\right)
$$

where $f_{j}(r):=\left(\bar{\gamma}(r)+\left|\mu_{j}\right|\right)\left|\varphi_{j}(r)\right|$, applying Lemma 32 (2) to $r \mapsto \varphi_{j}(r)-\varphi_{j}(R)$, we deduce that $\left\{\varphi_{j}\right\}_{j \in \mathbb{N}}$ is bounded in $W_{\mathrm{r}}^{2, q}(0, R)$, and we may assume by passing to a subsequence if necessary that it is convergent weakly in $W_{\mathrm{r}}^{2, q}(0, R)$ to a function $\varphi \in$ $W_{\mathrm{r}}^{2, q}(0, R)$, which implies that $\left\{\varphi_{j}\right\}$ converges to $\varphi$ in $C([0, R])$ and $\|\varphi\|_{L^{\infty}(0, R)}=1$.

Now, we apply Lemma 36 , with $f \equiv 0$, to see that $(\mu, \varphi)$ is an $n$th order eigenpair of (7) and (8).

Outline of proof of Corollary 8 The existence of $n$th order eigenpairs $\left(\mu^{ \pm}, \varphi^{ \pm}\right)$of (7) and (19) satisfying condition (1) can be shown by applying Theorem 7 to (7)-(8), with $\theta_{1}$ or $\theta_{2}$ in place of $\theta$. Given an $n$th order eigenpair $(\mu, \varphi)$ of (7) and (19), with $\|\varphi\|_{L^{\infty}(0, R)}=1$, it is clear that if $\varphi(0)>0$, then $(\mu, \varphi)=\left(\mu^{+}, \varphi^{+}\right)$and otherwise, $(\mu, \varphi)=\left(\mu^{-}, \varphi^{-}\right)$.

Outline of proof of Theorem 11 The proof of claims (2) and (3) is similar to that of the corresponding claims of Theorem 6 thanks to Theorem 34 .

For claim (1), recalling the proof of Theorem 7, we may select a sequence $\left\{a_{j}\right\}_{j \in \mathbb{N}} \subset$ $(0, R / 2)$ so that $a_{j} \rightarrow 0$ and $\mu_{\mathrm{r}}^{n}\left(\theta^{-}, \theta, a_{j}, R\right) \rightarrow \mu_{\mathrm{r}}^{n}(\theta, R)$, where $\theta^{-} \in\{\pi / 2,3 \pi / 2\}$ is chosen so that $\left(n, \theta^{-}, \theta\right)$ is admissible. Since $\mu<\mu_{\mathrm{r}}^{n}(\theta, R)$, we may assume $\mu<\mu_{\mathrm{r}}^{n}\left(\theta^{-}, \theta, a_{j}, R\right)$ for all $j \in \mathbb{N}$. Thus by Theorem 6 , there exists an $n$th order solution $u_{j} \in W_{\mathrm{r}}^{2, q}(0, R)$ of

$$
\mathcal{F}\left[u_{j}\right]+\mu u_{j}+\operatorname{sgn}\left(u_{j}\right) f=0 \quad \text { in } \quad\left(a_{j}, R\right), \quad B\left(u_{j}, a_{j}, R\right) \in L\left(\theta^{-}, \theta\right) .
$$


Since both $\pm u_{j}$ satisfy

$$
\mathcal{P}^{+}[u]+\bar{\beta}\left|u^{\prime}\right|+(\bar{\gamma}+|\mu|)|u|+f \geq 0 \quad \text { in }\left(a_{j}, R\right),
$$

once we show that $\left\{u_{j}\right\}$ is bounded in $L^{\infty}(0, R)$, thanks to Lemmas 32 and 36 , the existence of $n$th order solution of (26) follows.

We show that $\left\{u_{j}\right\}_{j \in \mathbb{N}}$ is bounded in $L^{\infty}(0, R)$. To the contrary, we suppose $\left\|u_{j}\right\|_{L^{\infty}(0, R)} \rightarrow \infty$ and set $v_{j}(r):=u_{j}(r) /\left\|u_{j}\right\|_{L^{\infty}(0, R)}$. Then $v_{j}$ satisfies

$$
\left\|v_{j}\right\|_{L^{\infty}(0, R)}=1, \quad \mathcal{F}\left[v_{j}\right]+\mu v_{j}+\frac{\operatorname{sgn}\left(u_{j}\right) f}{\left\|u_{j}\right\|_{L^{\infty}(0, R)}}=0 \quad \text { in }\left(a_{j}, R\right) .
$$

Arguing as above, using Lemmas 32 and 36 and the fact that $\operatorname{sgn}\left(u_{j}\right) f /\left\|u_{j}\right\|_{L^{\infty}(0, R)} \rightarrow$ 0 strongly in $L_{\mathrm{r}}^{q}(0, R)$, we may find an $n$th order eigenpair $(\mu, v) \in \mathbb{R} \times W_{\mathrm{r}}^{2, q}(0, R)$. However, since $\mu<\mu_{\mathrm{r}}^{n}(\theta, R)$, this is a contradiction. Thus $\left\{u_{j}\right\}$ is bounded in $L^{\infty}(0, R)$ and the proof is complete.

\section{Examples}

\subsection{Non-uniqueness for (18)}

We present examples of (18) that have many first order solutions.

Let $\Omega$ be the interval $(0,3)$ and consider the boundary value problem

$$
F[u]+\mu u+\operatorname{sgn}(u) f=0 \quad \text { in }(0,3) \quad \text { and } \quad B(u, 0,3) \in L(\pi / 2,3 \pi / 2)
$$

where $\mu$ is a constant, the function $F$ is given by $F(m, p, u, x)=m, f:=$ $\chi_{(0,1)}+\chi_{(2,3)}$ and $\chi_{(c, d)}$ denotes the characteristic function of the interval $(c, d)$. The boundary condition in (80) is of the Neumann type.

For $t \in[1 / 2,3 / 2]$ we define the function $u_{t}:[0,3] \rightarrow \mathbb{R}$ by

$$
u_{t}(x)= \begin{cases}t-\frac{1}{2} x^{2} & \text { for } x \in[0,1] \\ t-\frac{1}{2}-x+1 & \text { for } x \in[1,2] \\ t-2+\frac{1}{2}(x-3)^{2} & \text { for } x \in[2,3] \text { (Fig. 5). }\end{cases}
$$

Fig. 5 The graph of the function $u_{1}$

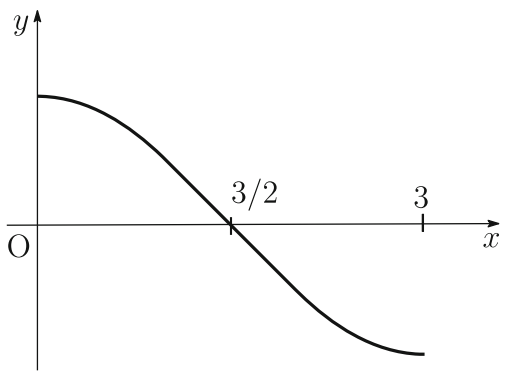


It is easily seen that $u_{t} \in W^{2, \infty}(0,3)$ and $u_{t}$ is a first order solution of (80) for $\mu=0$. By Theorem 6 , we see that $0<\mu^{1}(\pi / 2,3 \pi / 2,0,3)$. Thus, the family $\left\{u_{t}: t \in[1 / 2,3 / 2]\right\}$ tells us that the uniqueness of the first order solutions of (80) does not hold.

\subsection{Non-uniqueness for (26)}

We treat the radial case and show that a simple modification of the previous example yields an example of (26) that has many first order solutions.

Let $R=5$, define the functions $F$ and $f$ by

$$
F(M, p, u, x):=\operatorname{tr} M-\chi_{1,5}(x) \frac{N-1}{|x|}\left\langle\frac{x}{|x|}, p\right\rangle+\frac{\pi^{2}}{4} \chi_{4,5}(x) u
$$

and $f:=\chi_{1,2}+\chi_{3,4}$ where $\chi_{i, j}$ denotes the characteristic function of the annulus $\left\{x \in \mathbb{R}^{N}: i \leq|x| \leq j\right\}$, and consider the boundary value problem for $u \in$ $W_{\mathrm{r}}^{2, q}(0, R)$ :

$$
F\left(D^{2} u, D u, u, x\right)+\mu u+\operatorname{sgn}(u) f=0 \quad \text { in } \quad B_{R} \quad \text { and } \quad B^{+}(u, R) \in l(2 \pi),
$$

where $q>N / 2$ and $\mu$ are constants. Here the boundary condition is of the Dirichlet type. This problem can be rewritten as

$$
\mathcal{F}\left(u^{\prime \prime}, u^{\prime}, u, r\right)+\mu u+\operatorname{sgn}(u) g=0 \quad \text { in }(0,5) \quad \text { and } \quad B^{+}(u, 5) \in l(2 \pi),
$$

where $\mathcal{F}$ and $g$ are the functions given by

$\mathcal{F}(m, p, u, r):=m+\frac{N-1}{r} \chi_{(0,1)}(r) p+\frac{\pi^{2}}{4} \chi_{(4,5)}(r) u \quad$ and $\quad g:=\chi_{(1,2)}+\chi_{(3,4)}$.

For $t \in[1 / 2,3 / 2]$, we define the function $v_{t}:[0,5] \rightarrow \mathbb{R}$ by

$$
v_{t}(r)= \begin{cases}t & \text { for } r \in[0,1] \\ t-\frac{1}{2}(r-1)^{2} & \text { for } r \in[1,2] \\ t-\frac{1}{2}-r+2 & \text { for } r \in[2,3] \\ t-2+\frac{1}{2}(r-4)^{2} & \text { for } r \in[3,4] \\ (2-t) \sin (\pi(r-5) / 2) & \text { for } r \in[4,5] \text { (Fig. 6). }\end{cases}
$$

It is easily checked that this function $v_{t}$ belongs to $W_{\mathrm{r}}^{2, \infty}(0,5)$ and satisfies $(81)$ with $\mu=0$. Hence, the uniqueness for first order solutions of (81) does not hold in general. 


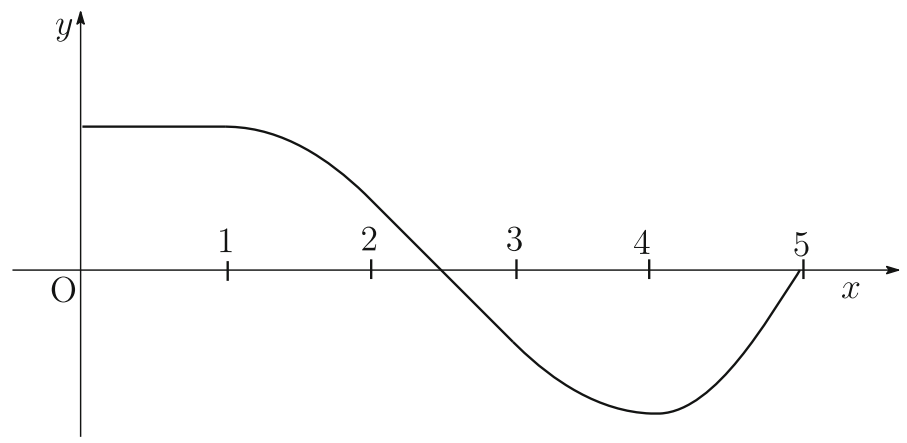

Fig. 6 The graph of the function $v_{1}$

Acknowledgments The authors would like to thank Professors Kazuhiro Ishige and Futoshi Takahashi for drawing the authors' attention to this problem as well as the Ref. [1]. The part of this paper was written while the first author was visiting the University of Warwick. He would like to thank the university for its hospitality and support.

Open Access This article is distributed under the terms of the Creative Commons Attribution 4.0 International License (http://creativecommons.org/licenses/by/4.0/), which permits unrestricted use, distribution, and reproduction in any medium, provided you give appropriate credit to the original author(s) and the source, provide a link to the Creative Commons license, and indicate if changes were made.

\section{References}

1. Amann, H.: Maximum principles and principal eigenvalues. Ten mathematical essays on approximation in analysis and topology, pp. 1-60, Elsevier, Amsterdam (2005)

2. Anane, A.: Simplicité et isolation de la premiére valeur propre du p-laplacien avec poids. C. R. Acad. Sci. Paris Sér. I Math. 305(16), 725-728 (1987)

3. Armstrong, S.N.: Principal eigenvalues and an anti-maximum principle for homogeneous fully nonlinear elliptic equations. J. Differ. Equ. 246(7), 2958-2987 (2009)

4. Berestycki, H.: On some nonlinear Sturm-Liouville problems. J. Differ. Equ. 26(3), 375-390 (1977)

5. Berestycki, H., Nirenberg, L., Varadhan, S.R.S.: The principal eigenvalue and maximum principle for second-order elliptic operators in general domains. Commun. Pure Appl. Math. 47(1), 47-92 (1994)

6. Birindelli, I., Demengel, F.: Comparison principle and Liouville type results for singular fully nonlinear operators. Ann. Fac. Sci. Toulouse Math. 13(2), 261-287 (2004)

7. Birindelli, I., Demengel, F.: First eigenvalue and maximum principle for fully nonlinear singular operators. Adv. Differ. Equ. 11(1), 91-119 (2006)

8. Birindelli, I., Demengel, F.: Eigenvalue, maximum principle and regularity for fully non linear homogeneous operators. Commun. Pure Appl. Anal. 6(2), 335-366 (2007)

9. Birindelli, I., Demengel, F.: The Dirichlet problem for singular fully nonlinear operators. Discrete continuous dynamic systems, dynamical systems and differential equations. In: Proceedings of the 6th AIMS International Conference, suppl., pp. 110-121 (2007)

10. Birindelli, I., Demengel, F.: Eigenvalue and Dirichlet problem for fully-nonlinear operators in nonsmooth domains. J. Math. Anal. Appl. 352(2), 822-835 (2009)

11. Birindelli, I., Demengel, F.: Uniqueness of the first eigenfunction for fully nonlinear equations: the radial case. Z. Anal. Anwend. 29(1), 77-90 (2010)

12. Birindelli, I., Demengel, F.: Regularity and uniqueness of the first eigenfunction for singular fully nonlinear operators. J. Differ. Equ. 249(5), 1089-1110 (2010)

13. Busca, J.: Existence results for Bellman equations and maximum principles in unbounded domains. Commun. Partial Differ. Equ. 24(11-12), 2023-2042 (1999) 
14. Busca, J., Esteban, M.J., Quaas, A.: Nonlinear eigenvalues and bifurcation problems for Pucci's operators. Ann. Inst. H. Poincaré Anal. Non Linéairé 22(2), 187-206 (2005)

15. Caffarelli, L.A.: Interior $W^{2, p}$ estimates for solutions of the Monge-Ampere equation. Ann. of Math. 131(1), 135-150 (1990)

16. Caffarelli, L.A., Cabré, X.: Fully Nonlinear Elliptic Equations. American Mathematical Society, Providence (1995)

17. Dancer, E.N.: On the Dirichlet problem for weakly non-linear elliptic partial differential equations. In: Proceedings of Royal Society Edinburgh Section A, vol. 76, no. 4, pp. 283-300 (1976/77)

18. del Pino, M., Manásevich, R.: Global bifurcation from the eigenvalues of the $p$-Laplacian. J. Differ. Equ. 92(2), 226-251 (1991)

19. Demengel, F.: Generalized eigenvalues for fully nonlinear singular or degenerate operators in the radial case. Adv. Differ. Equ. 14(11-12), 1127-1154 (2009)

20. Escauriaza, L.: $W^{2, n}$ a priori estimates for solutions to fully non-linear equations. Indiana Univ. Math. J. 42, 413-423 (1993)

21. Esteban, M.J., Felmer, P., Quaas, A.: Eigenvalues for radially symmetric fully nonlinear operators. Commun. Partial Differ. Equ. 35(9), 1716-1737 (2010)

22. Felmer, P., Valdebenito, D.: Eigenvalues for radially symmetric fully nonlinear singular or degenerate operators. Nonlinear Anal. 75(18), 6524-6540 (2012)

23. Fok, P.: Some maximum principles and continuity estimates for fully nonlinear elliptic equations of second order. Thesis (Ph.D.) UCSB, p. 82 (1996)

24. Fučik, S.: Boundary value problems with jumping nonlinearities. C̆asopis Pĕst. Mat. 101(1), 69-87 (1976)

25. Ikoma, N., Ishii, H.: Eigenvalue problem for fully nonlinear second-order elliptic PDE on balls. Ann. Inst. H. Poincaré Anal. Non Linéaire 29, 783-812 (2012)

26. Ishii, H., Yoshimura, Y.: Demi-eigenvalues for uniformly elliptic Isaacs operators, preprint

27. Koike, S., Swięch, A.: Weak Harnack inequality for fully nonlinear uniformly elliptic PDE with unbounded ingredients. J. Math. Soc. Japan 61(3), 723-755 (2009)

28. Lions, P.-L.: Bifurcation and optimal stochastic control. Nonlinear Anal. 7(2), 177-207 (1983)

29. Nadirashvili, N., Vlăduţ, S.: On axially symmetric solutions of fully nonlinear elliptic equations. Math. Z. 270(1-2), 331-336 (2012)

30. Patrizi, S.: Principal eigenvalues for Isaacs operators with Neumann boundary conditions. Nonlinear Differ. Equ. Appl. 16(1), 79-107 (2009)

31. Quaas, A., Sirakov, B.: Principal eigenvalues and the Dirichlet problem for fully nonlinear elliptic operators. Adv. Math. 218(1), 105-135 (2008)

32. Sirakov, B.: Solvability of uniformly elliptic fully nonlinear PDE. Arch. Ration. Mech. Anal. 195(2), 579-607 (2010) 